\title{
Robust Control for Bilateral Teleoperators with Soft Environments
}

\author{
by \\ Xiaoguang Wang, B. Eng. \\ A thesis submitted to \\ The Faculty of Graduate Studies and Research \\ in partial fulfillment of \\ the requirements for the degree of \\ Master of Applied Science in Electrical Engineering
Ottawa-Carleton Institute for Electrical and Computer Engineering
Department of Systems and Computer Engineering
Carleton University
Ottawa, Ontario, Canada \\ November 08,2005 \\ (C) Copyright \\ 2005, Xiaoguang Wang
}




$\begin{array}{ll}\begin{array}{l}\text { Library and } \\ \text { Archives Canada }\end{array} & \begin{array}{l}\text { Bibliothèque et } \\ \text { Archives Canada }\end{array} \\ \begin{array}{l}\text { Published Heritage } \\ \text { Branch }\end{array} & \begin{array}{l}\text { Direction du } \\ \text { Patrimoine de l'édition }\end{array} \\ \begin{array}{l}\text { 395 Wellington Street } \\ \text { Ottawa ON K1A ON4 }\end{array} & \begin{array}{l}\text { 395, rue Wellington } \\ \text { Ottawa ON K1A ON4 } \\ \text { Canada }\end{array}\end{array}$

Your file Votre référence

ISBN: 0-494-13452-6

Ourfile Notre référence

ISBN: 0-494-13452-6

NOTICE:

The author has granted a nonexclusive license allowing Library and Archives Canada to reproduce, publish, archive, preserve, conserve, communicate to the public by telecommunication or on the Internet, loan, distribute and sell theses worldwide, for commercial or noncommercial purposes, in microform, paper, electronic and/or any other formats.

The author retains copyright ownership and moral rights in this thesis. Neither the thesis nor substantial extracts from it may be printed or otherwise reproduced without the author's permission.
AVIS:

L'auteur a accordé une licence non exclusive permettant à la Bibliothèque et Archives Canada de reproduire, publier, archiver, sauvegarder, conserver, transmettre au public par télécommunication ou par l'Internet, prêter, distribuer et vendre des thèses partout dans le monde, à des fins commerciales ou autres, sur support microforme, papier, électronique et/ou autres formats.

L'auteur conserve la propriété du droit d'auteur et des droits moraux qui protège cette thèse. $\mathrm{Ni}$ la thèse ni des extraits substantiels de celle-ci ne doivent être imprimés ou autrement reproduits sans son autorisation.
In compliance with the Canadian

Privacy Act some supporting forms may have been removed from this thesis.

While these forms may be included in the document page count, their removal does not represent any loss of content from the thesis.
Conformément à la loi canadienne sur la protection de la vie privée, quelques formulaires secondaires ont été enlevés de cette thèse.

Bien que ces formulaires aient inclus dans la pagination, il n'y aura aucun contenu manquant.

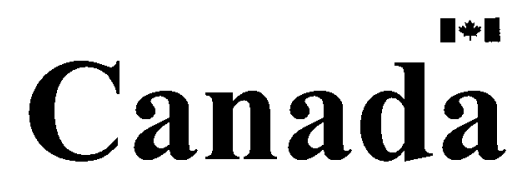




\section{Abstract}

Stability and fidelity are two key issues of bilateral teleoperator design. Previous work concentrates on situations where the remote environment is stiff and the interaction between the slave manipulator and environment is metal-to-metal. As a result, stability is usually the dominating issue in previous work. For applications like telesurgery, the manipulated objects, e.g. human body, organs and tissues, are however soft; so it is reasonable to expect that the stability problem in these cases becomes relatively less rigid while improving the fidelity, or in other words, the transparency of bilateral teleoperators, is significant. This thesis mainly studies how to improve system fidelity under the stability constraint. Specifically, the system design was explicitly formulated as an

optimization problem. A new fidelity measure combined with a robust control method is used to achieve better fidelity. 


\section{Acknowledgements}

I would like to thank my thesis supervisor, Professor Peter X. Liu, for being an excellent thesis supervisor. His guidance and inspiration is vital to the completion of this thesis. He is always very kind and ready to help me. His research vision in teleoperation systems impressed me very much and contributed a lot for the idea formation of the work.

Many professors at Carleton University and University of Ottawa have helped me in my research by giving me course lectures and research advices. I am grateful to professor professor P. Payeur and professor H. M. Schwartz.

Another important factor in completing the thesis is my wife, Ying Shen. Her support and encouragement is always of great importance to me.

I would also like to thank Rong Qian, Weiyu Zheng, Hua Yang and Zhifeng Zhang. They are all graduate students of Professor Peter. X. Liu. I can always get advice and assistance from them throughout the research process. 


\section{Contents}

$\begin{array}{ll}\text { Abstract } & \text { ii }\end{array}$

Acknowledgement $\quad$ iii

Acceptance Sheet $\quad$ iv

Contents $\quad$ v

List of Figures viii

List of Symbols $\quad \mathrm{x}$

1 Introduction 1

1.1 General background 3

1.1.1 Typical Bilateral Teleoperator Model 3

$\begin{array}{lll}\text { 1.1.2 Telesurgery } & 4\end{array}$

1.1.3 System Design Problems 5

1.1.4 Transparency/Fidelity 5

1.1.5 Stability 8

1.2 Thesis Overview $\quad 9$

2 System Modeling 11

2.1 Two-Port Network Model 12

$\begin{array}{ll}\text { 2.2 Physical Model and Dynamics of one-DOF Systems } & 17\end{array}$

2.2.1 Physical Model 17 
2.2.3 System Loop Gain $\quad 23$

3. Fidelity and Stability Analysis 25

3.1 Controller Design Formulation 26

$\begin{array}{ll}3.2 & \text { Fidelity Measure } \\ & 26\end{array}$

3.3 Tracking in Free Space as a Constraint 29

$\begin{array}{ll}3.4 \text { Stability Analysis } & 30\end{array}$

3.4.1 Passivity Theory 31

3.4.2 Robust Control Theory 34

3.4.3 Robust Stability Constraint for SISO Systems 40

4. Case Studies 44

4.1 Design Flow 45

4.2 Position-Force Controlled Teleoperator (Case 1) 46

$\begin{array}{ll}\text { 4.2.1 Tracking Constraint } & 47\end{array}$

4.2.2 Robust Stability Analysis $\quad 49$

4.2.3 Fidelity Evaluation $\quad 53$

4.2.4 Design based on Passivity Theorem 56

$\begin{array}{ll}\text { 4.2.5 Fidelity Comparison } & 57\end{array}$

4.3 Modified Position-Force Controlled Teleoperator (Case 2) 63

4.3.1 Tracking Constraint 63

4.3.2 Robust Stability Analysis $\quad 65$

$\begin{array}{ll}\text { 4.3.3 Fidelity Evaluation } & 72\end{array}$ 
5. Conclusions 


\section{List of Figures}

1.1 Typical teleoperator structure 3

2.1 Hybrid two-port network model of bilateral teleoperators $\quad 12$

2.2 General four-channel teleoperator structure 16

$\begin{array}{ll}2.3 \text { Physical model of a one-DOF teleoperation system } & 17\end{array}$

$\begin{array}{ll}2.4 \text { Position-force based teleoperator structure } & 20\end{array}$

2.5 Modified position-force based teleoperator structure 22

3.1 Standard feedback configurations $\quad 35$

3.2 Internal stability analysis diagram 36

3.3 Typical behaviour of multiplicative uncertainty 38

$\begin{array}{ll}3.4 \text { Output multiplicative perturbed systems } & 39\end{array}$

3.5 Closed-loop system to be studied 41

4.1 Proposed design flow 45

4.2 Tracking performance constraint (Case 1) 48

4.3 Nominal closed-loop system poles (Case 1) 50

4.4 Find the bounding function (Case 1) 51

4.5 Internal stability function (Case 1) 53

4.6 Bode diagram of $l(s) \quad 55$ 
4.7 Fidelity function (Case 1) 55

4.8 Passivity analysis (Case 1) 56

4.9 Fidelity Comparison (Case 1) 62

4.10 Tracking performance constraint (Case 2) 64

4.11 Nominal closed-loop system poles (Case 2) 66

4.12 Find the bounding function (Case $2 \alpha \in[0,0.5]$ ) 68

4.13 Find the bounding function (Case $2 \alpha \in(0.5,1])$

4.14 Internal stability criterion (Case 2, $\alpha \in[0,0.5]$ ) 71

4.15 Internal stability criterion (Case 2, $\alpha \in(0.5,1])$

$\begin{array}{ll}4.16 \text { Internal stability region } & 72\end{array}$

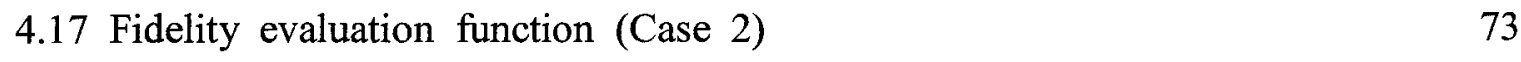

$\begin{array}{ll}4.18 \text { Passivity analysis (Case 2) } & 75\end{array}$

$\begin{array}{ll}4.19 \text { Passive region (Case 2) } & 75\end{array}$

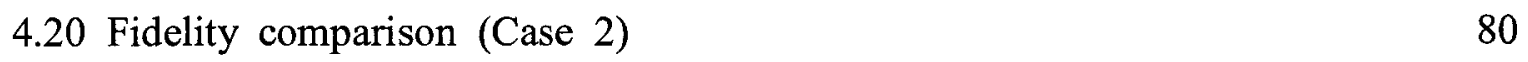




\section{List of Symbols}

The symbols used in the teleoperation field vary significantly. In this thesis, we try to apply the most commonly used ones and keep the consistency. The following symbols are used throughout this thesis. The definition of each symbol is given on the page indicated. If there is not ambiguity, we may drop the arguments, for example, write $H$ for $H(s)$ and $x$ for $x(t)$.

$\begin{array}{llll}b_{e} & 17 & G_{0}(s) & 41 \\ b_{h} & 17 & \hat{G}(s) & 49 \\ b_{m} & 17 & g & 20 \\ b_{s} & 17 & & \\ & & H(s) & 13 \\ D_{Z}(s) & 27 & H_{11}(s) & 13 \\ & & H_{12}(s) & 13 \\ e_{m}(s) & 32 & H_{21}(s) & 13 \\ e_{s}(s) & 32 & H_{22}(s) & 13 \\ F_{h}(s) & & H_{\infty} & 27 \\ F_{m}(s) & 12 & h(s) & 32 \\ F_{s}(s) & 12 & I & 33 \\ f_{h}(t) & 12 & & 58 \\ f_{m}(t) & 18 & K(s) & 17 \\ f_{s}(t) & 18 & k_{e} & 41 \\ G(s) & 18 & k_{e 0} & 17 \\ & & k_{h} & 17\end{array}$




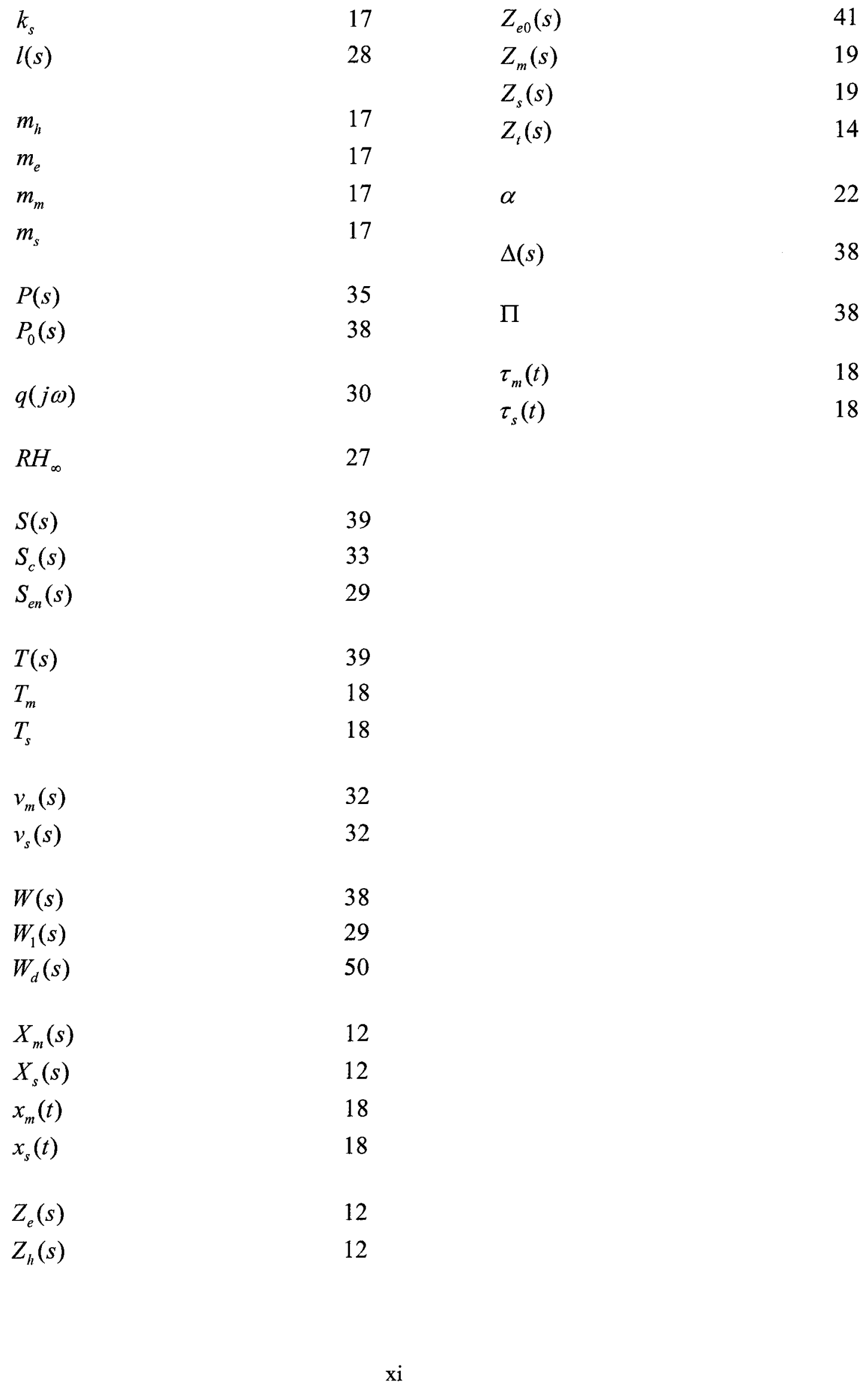




\section{Chapter 1}

\section{Introduction}

Teleoperators, or teleoperation systems, were first developed in the 1950's to handle nuclear materials [17]. The purpose of teleoperators is to enable human operators to perform tasks in environments that are remote, inaccessible or hazardous for human beings.

In the last decades, teleoperators have been found in a wide variety of applications including space explorations, undersea studies, surgery, mining, military operations, nuclear material handling, etc. Recently, new applications in medical training, personal communications, entertainment, and virtual reality are being explored [39]. With the rapid development of the Internet, teleoperators are being applied to many other fields and they are expected to have deeper impact on our daily life.

This thesis mainly focuses on a particular application, telesurgery, where surgeons perform surgical operations in remote locations far away from patients. The first 
telesurgery experiment was conducted between Italy and the USA in 1993 [38]. In the experiment, a biopsy was performed on a model containing animal organs. Such an integration of engineering and medicine offers great opportunities and hopes to many people living in isolated or rural areas. In recent years, many progresses in this field were reported [4] [16] [18] [29] [34] [40].

Different from traditional applications of teleoperation like nuclear material handling and space exploration, environments encountered in telesurgery are soft organs and tissues rather than stiff metal parts in space stations and nuclear plants. In the classical applications, the most important issue is to maintain the stability of the teleoperation system since the contact between the slave manipulator and the stiff environments can easily cause damage during manipulation if the system is unstable. However, in applications that involve the manipulation of soft objects, the stability constraint becomes less critical and improving the feedback fidelity of contact force between the slave robot and remote environment becomes significant.

In this thesis, the control design for bilateral teleoperators is explicitly formulated as an optimization process where controller parameters are optimized to minimize the newly proposed fidelity measure function. The new fidelity measure combined with a robust stability control method is applied to achieve better fidelity under the stability constraint. By applying this new design approach, higher fidelity is expected for teleoperators manipulating soft objects. 


\subsection{General Background}

\subsubsection{Typical Bilateral Teleoperator Model}

A typical teleoperator consists of three elements: a master manipulator which is manipulated by the human operator; a communication channel through which the control commands of the movements and forces of the master and slave are transmitted; a slave manipulator that performs required tasks in a remote or hazardous environment.

A typical bilateral teleoperator model can be depicted in Figure 1.1. The combined master-communication-slave block that is inside the dash lines is usually called MSN (master slave two-port network). In addition to $\mathrm{MSN}$, there are two elements that are very important in the teleoperator design, i.e., the human operator who commands and controls the remote task and the remote environment in which the task is performed.

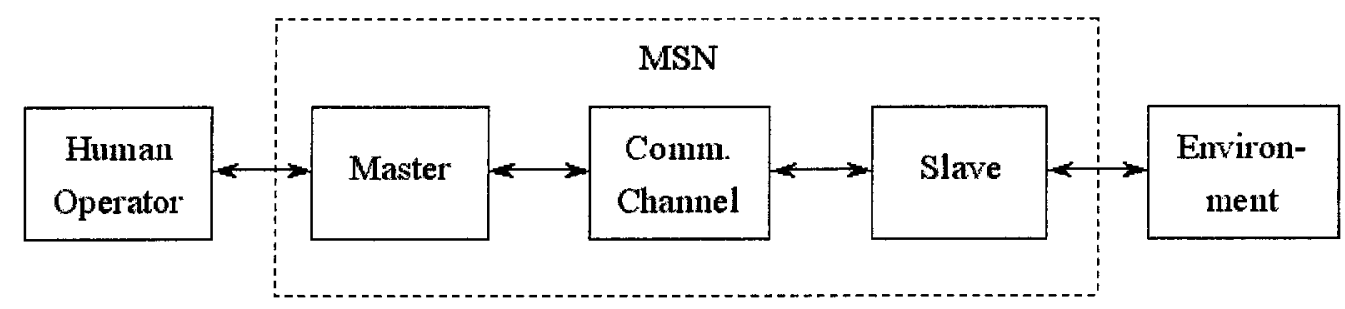

Figure 1.1: Typical teleoperator structure.

For a teleoperator, control commands are transmitted through the communication channel from the master side to the slave side. These commands make the remote manipulator perform desired tasks. To maintain safety and enhance performance, contact information between the slave and the environment is transmitted back to the human operator. This information can be presented to the human operator directly through 
mechanical signals on the master, or indirectly through visual or audio signals.

Video and audio information is essential for remote control systems. For some simple tasks, this indirect feedback is sufficient to achieve satisfactory performance and, at the same time, maintain stability. However, for complex tasks that require higher dexterity, the feedback of kinesthetic and tactile sensation is necessary.

In the case of force feedback, the contact information in the form of measured position or force signals is converted into mechanical signals by the master actuators and presented to the human operator. The human operator and environment are thus kinaesthetically coupled and the system control is bilateral since force information flows back and forward in both directions.

\subsubsection{Telesurgery}

In telesurgical applications, due to the physical separation, surgeons may lose the sensation and manipulation capabilities that are essential to the success of ordinary surgical operations. Teleoperation systems are designed to recover those lost capabilities. It is found that the direct force feedback from the slave to the master can increase the dexterity of the manipulation.

Unlike most traditional teleoperation applications, telesurgical tasks require very high dexterity and fidelity since surgeons largely rely on their kinaesthetic and tactile sensation to perform delicate manipulations and detect embedded tissues. Consequently, the design requirements for the teleoperator controllers are significantly different from those of traditional teleoperation applications. A very important issue in the telesurgery 
system design is the quantification of the human operator's sensitivity and system performance, which is necessary for the specification of the controller.

\subsubsection{System Design Problems}

The purpose of teleoperators is to extend a person's sensory and manipulative capability to a remote location [39]. In order to achieve satisfactory performance, a teleoperator needs to provide reliable and accurate information of the remote location to the human operator.

Performance and stability are two key issues of a teleoperator. Certainly, the performance of teleoperators depends heavily on the quality of the mechanical design of master and slave manipulators. However, the bilateral controller design plays a key role in determining the stability and transparency of the entire teleoperation system [41]. In previous works, the main concern of teleoperator design is usually force and motion tracking capability [48]. Except for performance, as a fundamental requirement for any control system, a teleoperator must be stable while it is performing tasks in an environment that normally has varying dynamic characteristics.

However, the performance and stability for a system cannot be improved simultaneously. In previous literature, the trade-offs between performance and stability were extensively studied [7] [10] [20] [28] [36].

\subsubsection{Transparency/Fidelity}

Like stability, system performance depends on system hardware design, the controller 
design and the task to be accomplished. In the literature, many performance measures have been proposed [35], e.g. sum of square forces [22] [27], manipulator dexterity and joint effort [43], maximum overshoot or minimum required bandwidth [5] [37], and transparency [8] [21] [28] [37] [42] [48].

Among the above performance measures, fidelity or transparency, defined as "the projection of human sensing and manipulation ability to remote locations" [39], fulfills the main objective of teleoperation. This is because the ultimate goal of teleoperators is to make the human operator feel as if he/she is directly manipulating the remote environment. If we regard the teleoperator as the interface between the human operator and the remote environment, the ideal design of the system is that the operator feels no interface at all.

Generally speaking, to achieve satisfactory performance, the teleoperator should be able to provide the human operator force feedback within the human operator's input bandwidth (tactile $300 \mathrm{~Hz}$, kinaesthetic $20 \mathrm{~Hz}$ ) and send the operator's output (around $5 \mathrm{~Hz}$ ) [5] to the remote environment faithfully. Therefore, transparency is quantitatively defined as a match of positions and forces between the master and slave to realize the condition of satisfactory manipulation.

Although transparency is a fundamental indicator for a bilateral teleoperator, the performance specification of a particular application depends on specific system configurations and tasks to be accomplished [7] [23].

In traditional applications such as in space and nuclear industry, the manipulator contacts with stiff environment and the force feedback need not to be exactly the same as 
the actual contact force since the force feedback itself is usually enough for the human operator to determine whether something unsafe has happened. In fact, in some applications, dynamic similarity is not desirable and the contact force needs to be scaled. For example, in micro/macro manipulations, the master position and force are usually scaled down/up at the slave side [25]. In telesurgery, it is sometimes more desirable to detect changes in the environment impedance than simple position and force tracking [7]. In [33], the performance of bilateral teleoperators is defined as the energy applied by the human operator to finish a given task.

Although there may be other design goals in teleoperation systems, in this thesis, we only consider the case where there is no scale up/down and transparency is the ultimate goal. Actually, with some slight modifications, our design method can be still effective when we use other performance measures.

Lawrence [28] first proposed the transparency conditions for bilateral teleoperators. The definition of transparency is based on the concept of impedance, i.e. a quantity that relates the input velocity to the output force of a system. For a mechanical system, the impedance depends on the mass, damping and stiffness of the system. Lawrence used the impedance ratio between the transmitted impedance and the environment impedance as a measure of transparency of teleoperation systems. Yokokohji et al. [48] defined three ideal responses, for which the goal of the control design was to match the positions and forces at the master and slave teleoperators. They proved that, if the conditions of three ideal responses were met, ideal transparency could be achieved.

The transparency concept proposed by Lawrence is widely accepted and 
acknowledged. Yokokohji et al. [48] proposed a measure of position and force tracking for the fidelity. However, their measures are only suitable for one degree-of-freedom (DOF) cases and the main objective of these two papers are to find a controller that can realize ideal position/force tracking rather than evaluate the performance of a particular teleoperator. In this thesis, we propose a new fidelity measure to evaluate the fidelity of teleoperators where the function is extended to multiple DOF cases.

\subsubsection{Stability}

Maintaining stability during the operation process is essential for any control system. The stability problem of teleoperators has been investigated using Lyapunov theory [41][42], passivity-based theory [3][6][28][36][48], Nyquist criterion [30], and root-locus technique [10][33].

Among the approaches, the passivity concept is probably the most widely used one. Passivity is also called unconditional stability in [48]. The stability of a passive system can be ensured when the system is coupled with any passive systems, environments or operators. However, this passivity condition is very restrictive since the range of arbitrary passive environments is too wide. The stability conditions achieved by passivity analysis are often quite conservative so that the performance is not good enough.

There are also some literature about using robust control theory for bilateral teleoperator design and analysis. In [25], Kazerooni et al. proposed an $H_{\infty}$ based framework to design a controller that only transmits force data. $H_{\infty}$ optimization was applied in [47] for motion scaling. Leung et al. [31] applied $\mu$ synthesis to bilateral 
teleoperator design with time delay. Cavusoglu [7] proposed that if a smaller set of environment and human operator impedance can be specified in the analysis, it is possible to increase the system fidelity under the constraint of stability.

\subsection{Thesis Overview}

This thesis deals with the design of bilateral teleoperators. In particular, it investigates the problem of improving the fidelity of teleoperators under the stability and other constraints using robust control design method. Parts of this thesis have been published and presented in my previous work [45] [46]. The rest of the thesis is organized as follows.

Chapter 2 introduces system modeling. First, the traditional two-port network representation of a teleoperation system is presented. Then the physical model of typical teleoperators is introduced. The chapter ends by a detailed dynamics analysis of two widely used bilateral teleoperator structures and the teleoperation system loop gain calculation.

Chapter 3 discusses the fidelity measure and stability analysis. First basic knowledge of $H_{\infty}$ norms is introduced. Then a new quantitative fidelity measure for bilateral teleoperators is proposed. The stability analysis methods are introduced and discussed in detail. Finally, the classical passivity conditions for stability and the robust stability for teleoperators are considered and compared.

Chapter 4 presents two case studies using the proposed robust control design approach. The steps that should be taken for the robust controller design are explained in detail. The results using the proposed approach and the traditional passivity approach are 
shown and compared.

Chapter 5 concludes this thesis and some future work is described. 


\section{Chapter 2}

\section{System Modeling}

Bilateral force-reflecting teleoperators is usually modeled as a two-port network $[1][3][7][8][20][21][28]$. The advantage of the two-port network exists in the fact that traditional electrical network theory can be used to analyze the system. In this case, the force and position/velocity variables of the teleoperation system correspond to the voltage and current in electrical systems. Because the electrical network theory is already quite mature, the analysis and simulations of teleoperators can be performed more easily. Until now, the network model is still the dominant one in this research field.

As mentioned in the first chapter, to model a teleoperation system, we need to consider five fundamental elements: the human operator, the environment, the master manipulator, the communication channel, and the slave manipulator. Besides, we need to model their connections. This is not a trivial work, especially when considering that the human operator is within the control loop. 


\subsection{Two-Port Network Model}

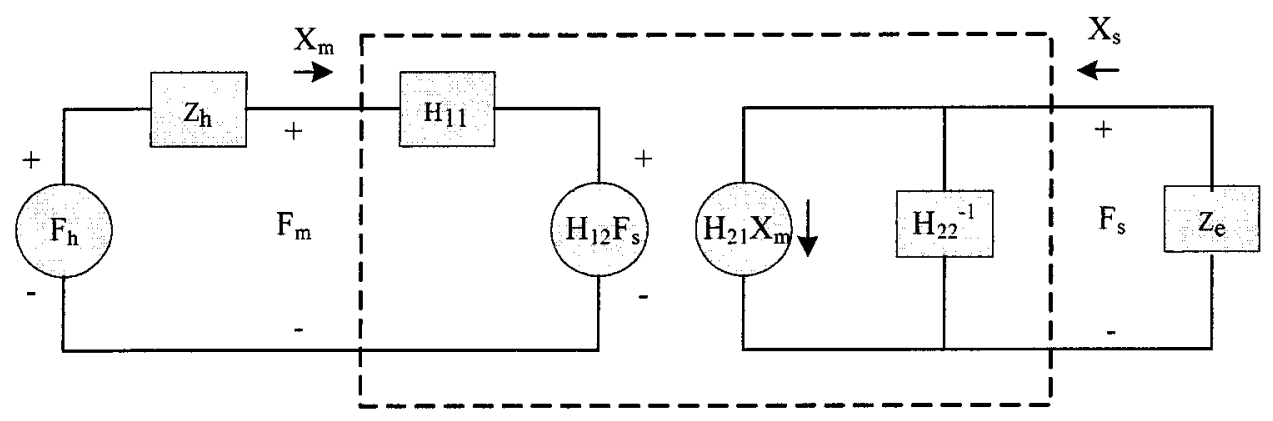

Figure 2.1: Hybrid two-port network model of bilateral teleoperators.

The hybrid two-port network model is actually a linear approximation of the system while the teleoperator is nonlinear in nature. According to Hannaford's representation [21], teleoperators can be described using a hybrid two-port network shown in Figure 2.1. Here, $F_{h}$ is the force applied by the human operator's muscle to the human's arm; $F_{m}$ is the force applied to the master manipulator by the human operator and $X_{m}$ is the position of the master; $F_{s}$ is the contact force between the slave manipulator and the environment and $X_{s}$ is the position of the slave; $Z_{h}$ and $Z_{e}$ are the human arm impedance and environment impedance respectively. These variables are all vectors so that we can deal with multiple DOF (degree of freedom) systems.

The dynamics of the above system can be expressed by the following hybrid two-port equation:

$$
\left[\begin{array}{l}
F_{m}(s) \\
X_{s}(s)
\end{array}\right]=\left[\begin{array}{ll}
H_{11}(s) & H_{12}(s) \\
H_{21}(s) & H_{22}(s)
\end{array}\right]\left[\begin{array}{c}
X_{m}(s) \\
F_{s}(s)
\end{array}\right]
$$

where the hybrid matrix is defined as 


$$
H(s)=\left[\begin{array}{ll}
H_{11}(s) & H_{12}(s) \\
H_{21}(s) & H_{22}(s)
\end{array}\right]
$$

The interpretation of the hybrid matrix $H(s)[21]$ is

$$
H=\left[\begin{array}{cc}
\text { Input Impedance } & \text { Force Scale } \\
\text { Position Scale } & \text { Output Admittance }
\end{array}\right]
$$

Consequently, the ideal hybrid matrix for a one DOF system is

$$
H_{\text {ideal }}=\left[\begin{array}{cc}
0 & 1 \\
-1 & 0
\end{array}\right]
$$

where the ideal force and position tracking is realized considering the opposite position definition of $X_{m}$ and $X_{s}$ in Figure 2.1.

For the two-port network model, there are also other representations depending on the different choices of inputs and outputs. Actually, for the four force and position variables $F_{m}, X_{m}, F_{s}$, and $X_{s}$ in the two-port network model, any two of them may be chosen as independent and the other two dependent.

If $X_{m}$ and $X_{s}$ are input variables, the system can be described by an impedance equation as:

$$
\left[\begin{array}{c}
F_{m}(s) \\
F_{s}(s)
\end{array}\right]=\left[\begin{array}{ll}
Z_{11}(s) & Z_{12}(s) \\
Z_{21}(s) & Z_{22}(s)
\end{array}\right]\left[\begin{array}{c}
X_{m}(s) \\
X_{s}(s)
\end{array}\right]
$$

If $F_{m}$ and $F_{s}$ are input variables, the system can be described by an admittance equation as:

$$
\left[\begin{array}{c}
X_{m}(s) \\
X_{s}(s)
\end{array}\right]=\left[\begin{array}{ll}
Y_{11}(s) & Y_{12}(s) \\
Y_{21}(s) & Y_{22}(s)
\end{array}\right]\left[\begin{array}{c}
F_{m}(s) \\
F_{s}(s)
\end{array}\right]
$$


If $F_{s}$ and $X_{s}$ are input variables, the system can be described by a transmission equation as:

$$
\left[\begin{array}{l}
F_{m}(s) \\
X_{m}(s)
\end{array}\right]=\left[\begin{array}{ll}
A_{11}(s) & B_{12}(s) \\
C_{21}(s) & D_{22}(s)
\end{array}\right]\left[\begin{array}{l}
F_{s}(s) \\
X_{s}(s)
\end{array}\right]
$$

Among these different representations the hybrid representation is widely investigated since it represents the case of position-error forward/force feedback structure, which is the most commonly used teleoperator structure in practical applications. In fact, these four different representations can be transformed easily between each other. In this thesis, we will focus on this hybrid representation.

Based on the two-port hybrid model shown in Figure 2.1, with $F_{h}$ being the original input signal to the system, we can derive the position and force responses of both master and slave manipulators. First, the relationship between the master force and position can be expressed as:

$$
F_{m}(s)=Z_{t}(s) X_{m}(s)
$$

where $Z_{t}$ is called the impedance transmitted by the teleoperator from the environment to the human operator. It can be shown [28] according to Figure 2.1 and equation (2.1.1) that

$$
Z_{i}(s)=H_{11}(s)-H_{12}(s) Z_{e}(s)\left[1+H_{22}(s) Z_{e}(s)\right]^{-1} H_{21}(s)
$$

From Figure 2.1 and equation (2.1.5), we have

$$
F_{h}(s)=Z_{h}(s) X_{m}(s)+F_{m}(s)=\left[Z_{h}(s)+Z_{t}(s)\right] \cdot X_{m}(s)
$$

Then it gives 


$$
\begin{aligned}
& X_{m}(s)=\left[Z_{h}(s)+Z_{t}(s)\right]^{-1} \cdot F_{h}(s) \\
& F_{m}(s)=Z_{t}(s) X_{m}(s)=Z_{t}(s) \cdot\left[Z_{h}(s)+Z_{t}(s)\right]^{-1} \cdot F_{h}(s)
\end{aligned}
$$

At the slave side, we also have the following equation from Figure 2.1,

$$
F_{s}(s)=-Z_{e}(s) X_{s}(s)
$$

Combining equations (2.1.1), (2.1.7) and (2.1.9), we have

$$
\begin{aligned}
X_{s}(s) & =H_{21}(s) X_{m}(s)+H_{22}(s) F_{s}(s) \\
& =H_{21}(s)\left[Z_{h}(s)+Z_{t}(s)\right]^{-1} F_{h}(s)-H_{22}(s) Z_{e}(s) X_{s}(s)
\end{aligned}
$$

Rearrange the above equation

$$
X_{s}(s)=\left[1+H_{22}(s) Z_{e}(s)\right]^{-1} \cdot H_{21}(s) \cdot\left[Z_{h}(s)+Z_{t}(s)\right]^{-1} \cdot F_{h}(s)
$$

And from equation (2.1.9) and (2.1.10), we have

$$
\begin{aligned}
F_{s}(s) & =-Z_{e}(s) X_{s}(s) \\
& =-Z_{e}(s)\left[1+H_{22}(s) Z_{e}(s)\right]^{-1} \cdot H_{21}(s) \cdot\left[Z_{h}(s)+Z_{t}(s)\right]^{-1} \cdot F_{h}(s)
\end{aligned}
$$

With the above equations, the four variables $F_{m}, X_{m}, F_{s}$, and $X_{s}$ can be calculated from the input $F_{h}$ directly. For an ideal teleoperator, the force and position tracking between master and slave are ideal, i.e. $F_{m}=F_{s}$ and $X_{m}=-X_{s}$. We can also evaluate the performance of a bilateral teleoperator by checking the force and position tracking errors between the master and slave [48]. In chapter 4 , we will validate our proposed design approach by checking these tracking errors for different input signals.

Besides the two-port network model, a four-channel model is shown in Figure 2.2 $[23][24][28][48]$ is also widely used. In this configuration, there are local control loops 
around the master and slave sides. The remote control loops are linked by four communication channels, which are labelled $C_{1}$ through $C_{4}$ in Figure 2.2. The four-channel model is an extension of the two-port network model for which both position and force information are transmitted bilaterally, which means more complexity and cost.

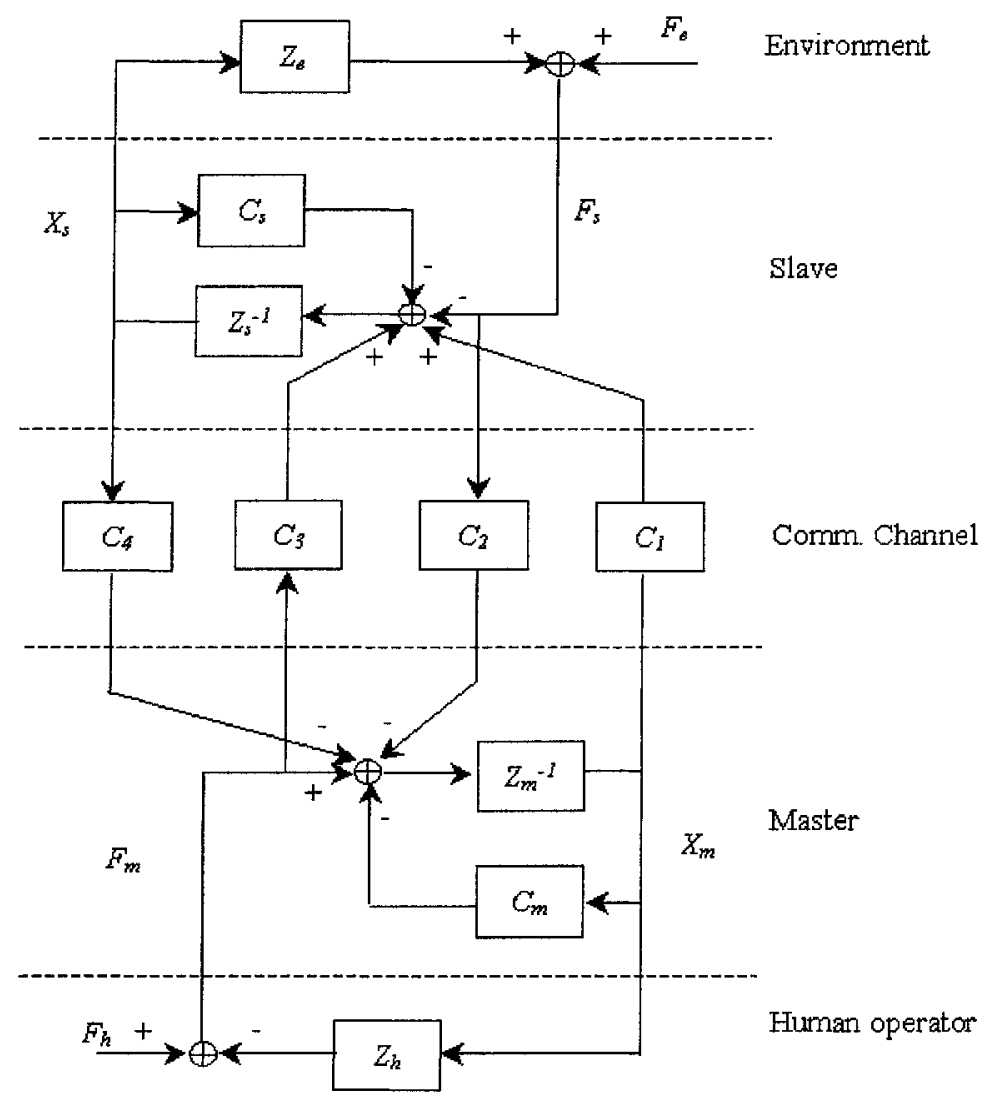

Figure 2.2: General four-channel teleoperator structure. 


\subsection{Physical Model and Dynamics for one DOF Systems}

\subsubsection{Physical Model}
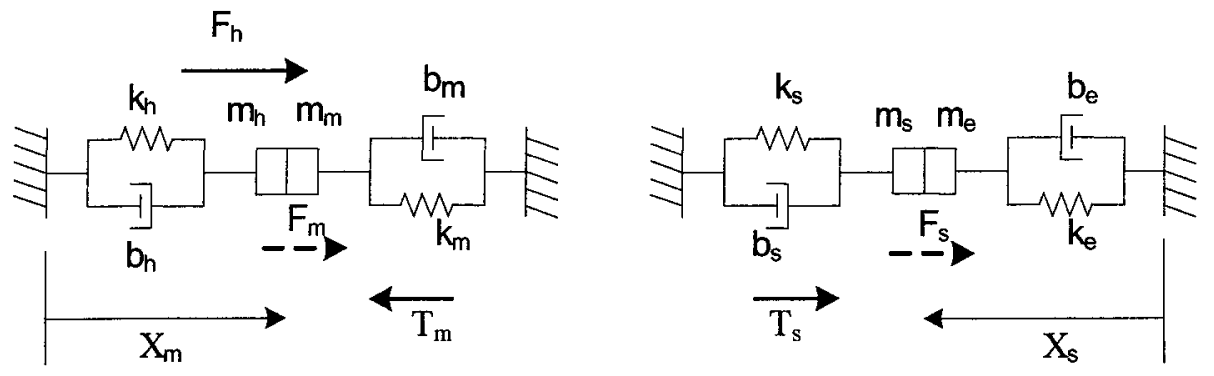

Figure 2.3: Physical model of a one-DOF teleoperation system.

In the previous section, the input and output variables $F_{m}, X_{m}, F_{s}$, and $X_{s}$ are vectors and the elements $H_{11}, H_{12}, H_{21}$ and $H_{22}$ of the hybrid matrix are transfer matrices. For simplicity, in this section, we will present detailed physical models and the dynamic analysis for one DOF teleoperation systems. These models and analysis will be used in the case studies in chapter 4 .

Figure 2.3 shows the physical model for one DOF teleoperators. The master and slave manipulators, the human operator, and the environments are all modeled as mechanical devices with masses, dampers and springs.

First, we define the notations in Figure 2.3 and these notations will be used throughout this thesis. $m_{m}, m_{s}, b_{m}, b_{s}, k_{m}$, and $k_{s}$ are the inertia, damping ratio, and stiffness of the master and slave manipulators respectively; $m_{h}, b_{h}$ and $k_{h}$ are the effective inertia, damping and stiffness of the human arm; $m_{e}, b_{e}$ and $k_{e}$ represent the 
inertia, damping and stiffness of the remote environment; In addition, $T_{m}$ and $T_{s}$ are the forces applied to the master and slave manipulators by the driving motors of master and slave controllers; $F_{m}, F_{s}, X_{m}, X_{s}$, and $F_{h}$ have already been defined in section 2.1. Since $F_{m}$ is the internal interacting force between $m_{m}$ and $m_{h}$, and $F_{s}$ is the internal interacting force between $m_{s}$ and $m_{e}$, these two internal forces were denoted in dashed line with arrows in Figure 2.3.

The dynamics of the master and slave manipulators of this physical model is given by the following equations. Note that the following four equations are in time domain. So, we use the notations $f_{h}, f_{m}, f_{s}, x_{m}, x_{s}, \tau_{m}$, and $\tau_{s}$ instead of $F_{h}, F_{m}, F_{s}, X_{m}$, $X_{s}, T_{m}$ and $T_{s}$ in these equations. For simplicity, the time variable $t$ is dropped.

$$
\begin{aligned}
& f_{m}-\tau_{m}=m_{m} \ddot{x}_{m}+b_{m} \dot{x}_{m}+k_{m} x_{m} \\
& f_{s}-\tau_{s}=m_{s} \ddot{x}_{s}+b_{s} \dot{x}_{s}+k_{s} x_{s}
\end{aligned}
$$

The dynamics of the human operator can also be modeled as a mass-damper-spring system. The dynamics equation is

$$
f_{h}-f_{m}=m_{h} \ddot{x}_{m}+b_{h} \dot{x}_{m}+k_{h} x_{m}
$$

Similarly, the dynamics of the environment is given by

$$
f_{s}=-\left(m_{e} \ddot{x}_{s}+b_{e} \dot{x}_{s}+k_{e} x_{s}\right)
$$

The above equations are in time domain. Rewriting equations (2.2.1) and (2.2.2) in frequency domain, we have

$$
F_{m}(s)-T_{m}(s)=\left(m_{m} s^{2}+b_{m} s+k_{m}\right) \cdot X_{m}(s)
$$




$$
F_{s}(s)-T_{s}(s)=\left(m_{s} s^{2}+b_{s} s+k_{s}\right) \cdot X_{s}(s)
$$

The impedances of master and slave manipulators are defined as follows,

$$
\begin{aligned}
& Z_{m}(s)=m_{m} s^{2}+b_{m} s+k_{m} \\
& Z_{s}(s)=m_{s} s^{2}+b_{s} s+k_{s}
\end{aligned}
$$

Substitute the above representations into equation (2.2.5) and (2.2.6), we have

$$
\begin{aligned}
& F_{m}(s)-T_{m}(s)=Z_{m}(s) \cdot X_{m}(s) \\
& F_{s}(s)-T_{s}(s)=Z_{s}(s) \cdot X_{s}(s)
\end{aligned}
$$

In frequency domain, equation (2.2.3) and (2.2.4) are transformed to

$$
\begin{aligned}
& F_{h}(s)-F_{m}(s)=\left(m_{h} s^{2}+b_{h} s+k_{h}\right) \cdot X_{m}(s) \\
& F_{s}(s)=-\left(m_{e} s^{2}+b_{e} s+k_{e}\right) \cdot X_{s}(s)
\end{aligned}
$$

The impedances of the human operator and the environment are defined as

$$
\begin{aligned}
& Z_{h}(s)=m_{h} s^{2}+b_{h} s+k_{h} \\
& Z_{e}(s)=m_{e} s^{2}+b_{e} s+k_{e}
\end{aligned}
$$

Combining equation (2.2.9) and (2.2.10) with the above impedance definitions, we have

$$
\begin{aligned}
& F_{h}(s)-F_{m}(s)=Z_{h}(s) \cdot X_{m}(s) \\
& F_{s}(s)=-Z_{e}(s) \cdot X_{s}(s)
\end{aligned}
$$

\subsubsection{Two Teleoperator Structures}

Based on the physical model of teleoperators, different control laws have been developed. 
In this subsection, two classical teleoperator control structures are introduced, i.e. position-force based structure and modified position-force based structure. In the following, their hybrid transfer matrices are derived and these two structures will be used in the case studies in chapter 4.

\section{Position-Force Based Teleoperators}

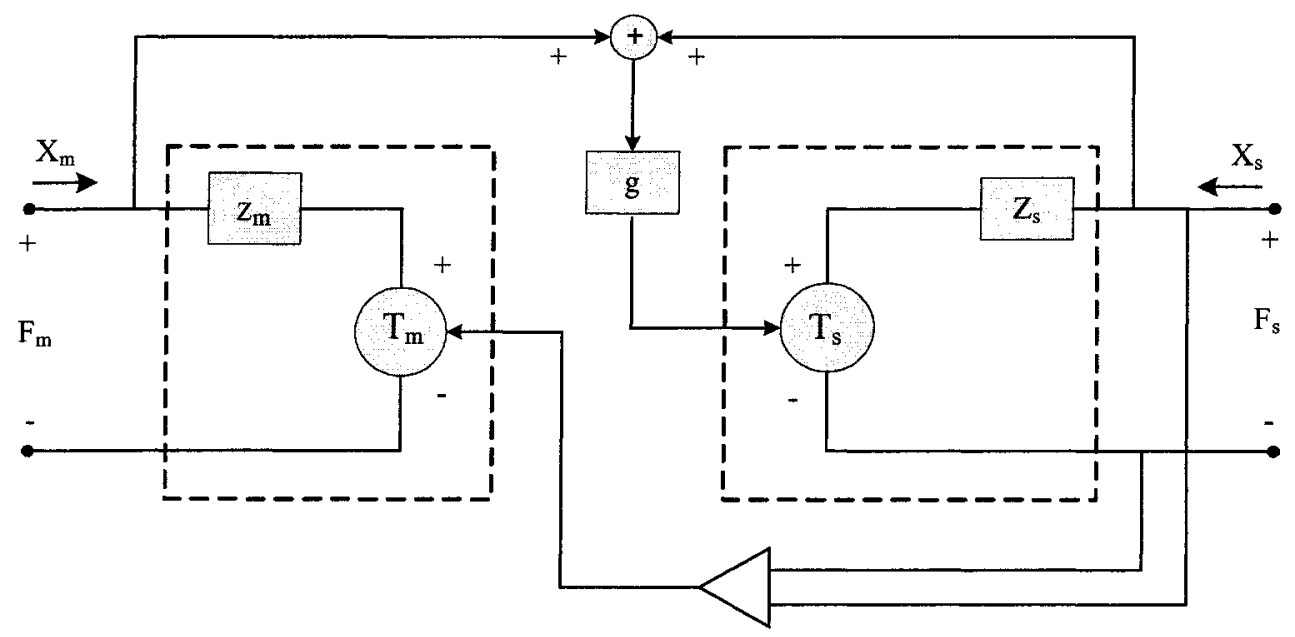

Figure 2.4: Position-force based teleoperator structure.

In order to achieve certain performance specifications of teleoperators, the contact force between the slave robot and the remote environment is directly transmitted back to the master side. Such position-force structure depicted in Figure 2.4 was widely accepted in the real applications. In the figure, $g$ is the forward position error gain and other notations follow the previous definitions.

In this system, the position error between the master and slave manipulators is used to control the slave manipulator and the measured force between the slave and the environment is directly applied to the master manipulator. 
The control law for such a structure is

$$
\begin{aligned}
& T_{m}(s)=F_{s}(s) \\
& T_{s}(s)=g \cdot\left[X_{m}(s)+X_{s}(s)\right]
\end{aligned}
$$

Substituting the above control laws into (2.2.7) and (2.2.8), we have

$$
\begin{aligned}
& F_{m}(s)-F_{s}(s)=Z_{m}(s) \cdot X_{m}(s) \\
& F_{m}(s)=Z_{m}(s) \cdot X_{m}(s)+F_{s}(s) \\
& F_{s}(s)-g \cdot\left[X_{m}(s)+X_{s}(s)\right]=Z_{s}(s) \cdot X_{s}(s) \\
& X_{s}(s)=-\frac{g}{Z_{s}(s)+g} \cdot X_{m}(s)+\frac{1}{Z_{s}(s)+g} F_{s}(s)
\end{aligned}
$$

Combining (2.2.15) and (2.2.16), and comparing them with the hybrid representation of equation (2.1.1), we get

$$
\begin{aligned}
& H_{11}(s)=Z_{m}(s) \\
& H_{12}(s)=1 \\
& H_{21}(s)=-\frac{g}{Z_{s}(s)+g} \\
& H_{22}(s)=\frac{1}{Z_{s}(s)+g}
\end{aligned}
$$

Considering the impedance definitions of $Z_{m}(s)$ and $Z_{s}(s)$, the hybrid matrix $H(s)$ of this position-force based architecture is given by

$$
H(s)=\left[\begin{array}{cc}
m_{m} s^{2}+b_{m} s+k_{m} & 1 \\
-\frac{g}{m_{s} s^{2}+b_{s} s+k_{s}+g} & \frac{1}{m_{s} s^{2}+b_{s} s+k_{s}+g}
\end{array}\right]
$$




\section{Modified Position-Force Based Teleoperators}

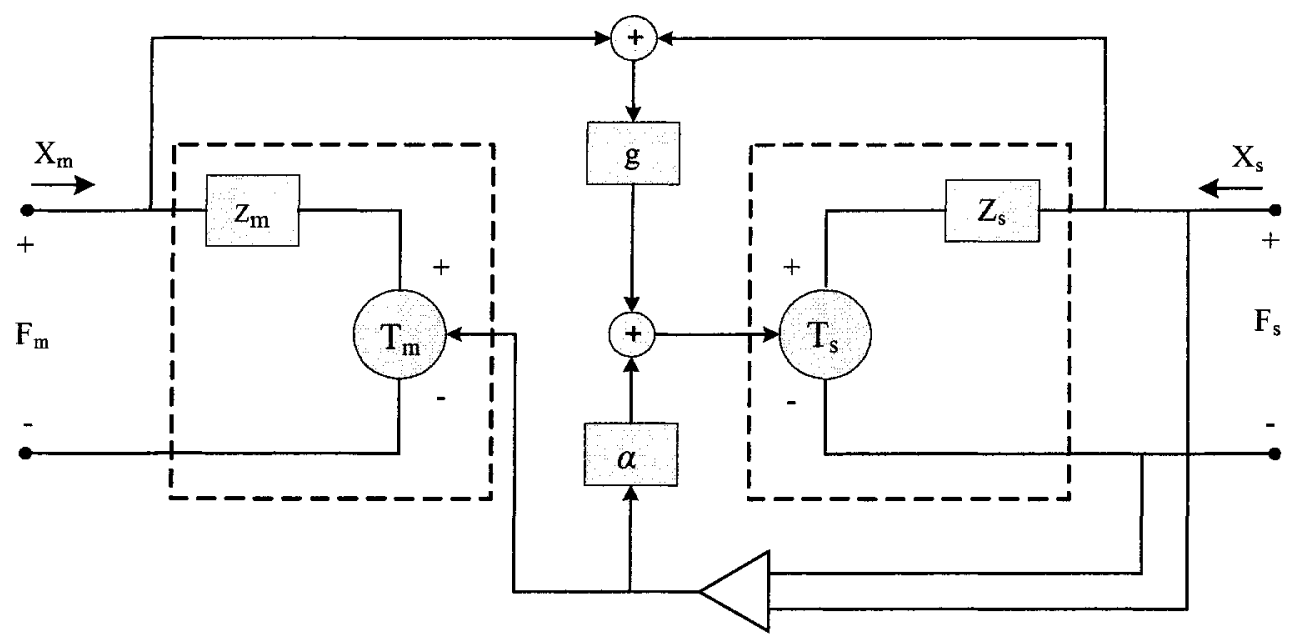

Figure 2.5: Modified position-force based teleoperator structure.

Although the position-force based structure shown in Figure 2.4 can provide direct force feedback to the human operator and achieve high dexterity in remote manipulation, the system usually has a relatively poor stability margin. To solve this problem, local force feedback at the slave side is proposed in [2][13][14][20][27].

In Figure 2.5, the modified position-force structure is depicted. In this configuration, $F_{s}$, the measured force between the slave and the environment is not only transmitted back to the master manipulator, but also partially fed back locally to the slave manipulator. This configuration has two controller parameters: one is the position error gain $g$ and the other is the local feedback gain $\alpha$, which is usually in the range $[0,1]$.

From Figure 2.5, the control law for such a modified position-force structure can be expressed as:

$$
T_{m}(s)=F_{s}(s)
$$




$$
T_{s}(s)=g \cdot\left[X_{m}(s)+X_{s}(s)\right]+\alpha \cdot F_{s}(s)
$$

Substituting (2.2.18) into (2.2.7) and (2.2.19) into (2.2.8), we have

$$
\begin{aligned}
& F_{m}(s)-F_{s}(s)=Z_{m}(s) \cdot X_{m}(s) \\
& F_{m}(s)=Z_{m}(s) \cdot X_{m}(s)+F_{s}(s) \\
& F_{s}(s)-g \cdot\left[X_{m}(s)+X_{s}(s)\right]-\alpha \cdot F_{s}(s)=Z_{s}(s) \cdot X_{s}(s) \\
& X_{s}(s)=-\frac{g}{Z_{s}(s)+g} \cdot X_{m}(s)+\frac{1-\alpha}{Z_{s}(s)+g} \cdot F_{s}(s)
\end{aligned}
$$

Comparing (2.2.20), (2.2.21) and the hybrid representation equation (2.1.1), we have

$$
\begin{aligned}
& H_{11}(s)=Z_{m}(s) \\
& H_{12}(s)=1 \\
& H_{21}(s)=-\frac{g}{Z_{s}(s)+g} \\
& H_{22}(s)=\frac{1-\alpha}{Z_{s}(s)+g}
\end{aligned}
$$

Considering the impedance definitions of $Z_{m}$ and $Z_{s}$, the hybrid matrix for the modified position-force configuration is

$$
H(s)=\left[\begin{array}{cc}
m_{m} s^{2}+b_{m} s+k_{m} & 1 \\
-\frac{g}{m_{s} s^{2}+b_{s} s+k_{s}+g} & \frac{1-\alpha}{m_{s} s^{2}+b_{s} s+k_{s}+g}
\end{array}\right]
$$

\subsubsection{System Loop Gain}

In this thesis, robust stability theory for the standard input-output feedback control system is applied instead of the traditional passivity theory. As a consequence, we need to 
derive the loop gain of the overall teleoperation system. This loop gain can be expressed through the hybrid matrix, so that we can conveniently convert network representations into classical frequency-domain representations.

From Figure 2.1, to calculate the loop gain, we can consider two cases, which can be combined by superposition [20].

For the first case, we can look at the forward path. It is assumed a position source is placed at the master port, which corresponds to $Z_{h}(s)=\infty$. From Figure 2.1, we have

$$
\frac{F_{s}(s)}{X_{m}(s)}=-H_{21}(s) \cdot\left[Z_{e}(s) \| \frac{1}{H_{22}(s)}\right]
$$

where,

$$
Z_{e}(s) \| \frac{1}{H_{22}(s)}=\frac{Z_{e}(s)}{1+Z_{e}(s) H_{22}(s)}
$$

For the second case, in the reverse path, we assume a force source is placed at the slave port, which corresponds to $Z_{e}=0$. It gives

$$
\frac{X_{m}(s)}{F_{s}(s)}=\frac{H_{12}(s)}{Z_{h}(s)+H_{11}(s)}
$$

The open loop gain is the product of the above two transfer functions. Combining equation (2.2.23) and (2.2.24), we have the loop gain function as follows:

$$
G(s)=\frac{-H_{12}(s) H_{21}(s) Z_{e}(s)}{\left(H_{11}(s)+Z_{h}(s)\right) \cdot\left(1+H_{22}(s) Z_{e}(s)\right)}
$$

If we can first find the hybrid matrix for the teleoperator and, at the same time, know the dynamics of the human operator and the environment, the system loop gain can be directly calculated according to equation (2.2.25). 


\section{Chapter 3}

\section{Fidelity and Stability Analysis}

For bilateral teleoperators, performance and stability are two primary design issues. Achieving the best performance under the constraint of stability is a fundamental requirement for teleoperator design. In this chapter, topics concerning performance and stability are discussed.

It is well known that there are many ways to define the performance of a bilateral teleoperator depending on the various requirements of applications. Among those measures, fidelity, or transparency, is the most commonly used one, which defines the closeness of the dynamics between the master and slave manipulators. In this chapter, we will propose a new numerical measure to accurately evaluate the fidelity of teleoperators.

For stability analysis of bilateral teleoperators, passivity theory is the most widely used method due to the dominance of the network theory in this field. However, the requirement of passivity is too conservative and system designs based on passivity theory 
normally cannot achieve satisfactory performance. In this thesis, robust stability theory is chosen as the framework to deal with the teleoperation system stability problem.

\subsection{Controller Design Formulation}

The controller design of bilateral teleoperators can be formulated as an optimization process [7]. In this thesis, we assume that the controller structure of the bilateral teleoperator is known and our task is to find controller parameters' values that can lead to the best fidelity.

We explicitly express the control design as: choosing controller parameters that minimize the fidelity measure function under the constraint of stability and free space tracking. That is

$$
\arg \min _{\text {stability and tracking constraint }}\left(D_{z}\right)
$$

where "arg" means the arguments, or, controller parameters in this case; $D_{z}$ is the new fidelity measure function which will be introduced in the next section, where smaller $D_{z}$ value corresponds to better fidelity.

\subsection{Fidelity Measure}

As mentioned in the first chapter, there are different definitions for the performance of a teleoperator due to the different purposes and requirements of specific applications. Finding an appropriate performance measure is of great importance for teleoperator design. 
In most of the literature, fidelity or transparency is the performance specification. For ideal transparency, the master and slave manipulator have exactly the same position and force at any given time. In this thesis, we will continue to use fidelity or transparency as the performance specification. If we take fidelity or transparency as the performance measure, the ultimate goal of teleoperators is to make the human operator feel like he/she is directly contacting with the remote environment.

Before we propose the new fidelity measure, we first give the definitions of $H_{\infty}$ space and $H_{\infty}$ norm.

\section{Definition 3.1}

If $A(s)$ is a transfer function matrix of a linear system, the class of systems for which $A$ is analytic and finite on the open right half plane is known as $H_{\infty}$ space. The $H_{\infty}$ norm is defined as

$$
\|A(s)\|_{\infty}:=\sup _{\operatorname{Re}(s)>0} \bar{\sigma}[A(s)]=\sup _{\omega \in R} \bar{\sigma}[A(j \omega)]
$$

where $R$ is the field of real numbers. $\bar{\sigma}(A)$ means the singular value of matrix $A$.

The second equality comes from the maximum modulus theorem for complex functions. Furthermore, the real rational subspace of $H_{\infty}$ is denoted by $R H_{\infty}$, which consists of all proper and real rational stable transfer matrices.

The new fidelity measure function is defined as:

$$
D_{z}\left(Z_{e}\right)=\left\|\left(Z_{i}(s)-Z_{e}(s)\right) \cdot l(s)\right\|_{\infty}
$$

where $Z_{t}$ is the impedance transmitted to the human operator through the teleoperator, 
which is already defined in equation (2.1.6); $Z_{e}$ is the environment impedance; $l(s)$ is a weighting factor putting more emphasis on the working low frequency range. This means that $l(s)$ is a low-pass filter with the frequency range within the human input bandwidth.

It should be noted that, since the impedance transfer functions $Z_{t}(s)$ and $Z_{e}(s)$ are generally not proper, we need to make sure that $\left(Z_{t}(s)-Z_{e}(s)\right) \cdot l(s)$ is proper when we choose $l(s)$. Otherwise, equation (3.2.1) will be meaningless.

From the hybrid two-port network model shown in Figure 2.1, the environment impedance transmitted to the human operator through the teleoperator has already been derived in equation (2.1.5) and (2.1.6), we have

$$
\begin{aligned}
& F_{m}(s)=Z_{t}(s) X_{m}(s) \\
& Z_{t}(s)=H_{11}(s)-H_{12}(s) Z_{e}(s)\left[\left(1+H_{22}(s)\right) Z_{e}(s)\right]^{-1} H_{21}(s)
\end{aligned}
$$

and from equation (2.1.9), the environment impedance is

$$
Z_{e}(s)=-F_{s}(s) X_{s}(s)^{-1}
$$

From equation (3.2.1), $D_{z}\left(Z_{e}\right)$ is actually the difference between the impedance 'felt' by the human operator at the master side and the actual environment impedance touched by the slave manipulator.

So, $D_{z}$ characterizes the divergence of the system to ideal transparency. In other words, if the value of $D_{z}$ is smaller, the force feedback is more faithful. If $D_{z}$ equals 0 , we have complete transparency. So our design goal is to choose appropriate controller 
parameters such that $D_{z}$ is minimized under the constraints of stability and free space tracking.

\subsection{Tracking in Free Space as a Constraint}

In the definition of fidelity, we know that the position scale between the master and slave should be unity. Because the impedance similarity cannot prevent us from getting some other scale factors, we need a constraint to prevent us from achieving non-unity scale in the control design. As in [7], we use free space position tracking between the master and slave as a constraint.

According to the two-port representation, i.e. equation (2.1.1), we know that

$$
X_{s}(s)=H_{21}(s) X_{m}(s)+H_{22}(s) F_{s}(s)
$$

In free space, it gives $F_{s}=0$. Thus we have

$$
-X_{s}(s)=-H_{21}(s) \cdot X_{m}(s)
$$

The transfer function from the reference input signal $X_{m}$ to the tracking error between $X_{m}$ and $-X_{s}$ (considering the different direction notations of $X_{m}$ and $X_{s}$ ) is the sensitivity function which can be obtained as

$$
S_{e n}(s)=1-\left[-H_{21}(s)\right]=1+H_{21}(s)
$$

We can find a specific transfer function $W_{1}(s)$ that gives a tracking performance specification. And the transfer function will satisfy this requirement if

$$
\left\|S_{e n}(s) W_{1}(s)\right\|_{\infty}<1
$$

For example, considering a SISO (single input single output) system, we can define 
a transfer function $q(s)$, which represents the requirement of less than $3 \%$ tracking error for any input signal with frequencies under $5 \mathrm{~Hz}$. The frequency response of such a function $q(j \omega)$ can be plotted on the Bode diagram.

And if the sensitivity function satisfies

$$
\left|S_{e n}(j \omega)\right|<|q(j \omega)|
$$

we can define

$$
W_{1}(j \omega)=\frac{1}{q(j \omega)}
$$

Then we know that if the inequality of (3.3.4) or (3.3.5) is satisfied, the free space tracking constraint is satisfied by our system.

\subsection{Stability Analysis}

The classical stability analysis tools for linear time invariant (LTI) systems such as pole-zero analysis, Nyquist criterion and root locus methods can be used to determine if the teleoperation system is stable. At the same time, energy based stability analysis tools such as Lyapunov theory and passivity theory can be used. There are also some literatures using $H_{\infty}$ and $\mu$ robust control method of modern control theory [7] [25] [26] [31] [47].

In this thesis, robust stability analysis is applied to guarantee the internal stability of bilateral teleoperators. However, since most people use passivity theory as the stability analysis tool, we will first introduce some fundamental knowledge of the passivity theory. 


\subsubsection{Passivity Theory}

Passivity theory is widely used in teleoperation systems' stability analysis. It is suitable for both linear and non-linear systems and is a very powerful method. Here, we discuss some primary results about the stability analysis based on passivity theory. Detailed treatment can be found in [11].

Passivity is a sufficient condition for stability of systems interacting with any passive environment. Colgate and Hogan [9] proposed that the sufficient and necessary stability condition for a bilateral teleoperation system interacting with any passive environment is that the system must be passive itself. Anderson and Spong [3] studied the stability of bilateral teleoperators with time delay based on the passivity theorem.

It should be noted that passivity itself is not a necessary and sufficient condition of stability. Passivity of a system can be a sufficient condition of stability only when the system interacts with passive environments, for example, a lossless passive system such as a simple mass-spring system may generate unbounded outputs by bounded inputs.

For bilateral teleoperators, if we assume that the human operator and the environment are passive systems, a sufficient condition of stability is that the master-slave system must be passive. However, the human operator is not passive at all because he/she has muscles that can generate power actively. Colgate and Hogan [9] solved this problem. They showed that even if the system has an active term, the system stability is guaranteed unless the active term is in some way state-dependent. The following assumption is proposed in [9]: the human operator's input is independent of the state of the master-slave system. In other words, the operator does not generate forces 
that will cause the system to be unstable. Dudragne [12] gave a similar assumption in order to apply the concept of passivity. Obviously, the above assumption is a little tricky and is opposed by some researchers, but it is necessary to ensure the system stability [48].

Some basic concepts of passivity theory are introduced in the following and the contents are based on [3] [11].

A system is determined by the relationship between effort $e$ (force or voltage), and the flow $v$ (velocity or current). A hybrid matrix similar to equation (2.1.1) can be used to represent the relationship between the effort and flow for a linear system,

$$
\left[\begin{array}{c}
e_{m}(s) \\
v_{s}(s)
\end{array}\right]=h(s)\left[\begin{array}{c}
v_{m}(s) \\
e_{s}(s)
\end{array}\right]=\left[\begin{array}{ll}
h_{11}(s) & h_{12}(s) \\
h_{21}(s) & h_{22}(s)
\end{array}\right]\left[\begin{array}{l}
v_{m}(s) \\
e_{s}(s)
\end{array}\right]
$$

where $e_{m}, e_{s}, v_{m}$ and $v_{s}$ are respectively the efforts and flows of the master and slave port.

\section{Definition 3.2}

An $n$-port is said to be passive if and only if for any independent set of $n$ port flows $v_{i}$ injected into the system, and efforts $e_{j}$ applied across the system

$$
\int_{0}^{t} e^{T}(\tau) v(\tau) d \tau=E(t)-E(0)+\int_{0}^{t} P_{d i s s} d \tau \geq-E(0)=\text { constant }
$$

where $e=\left[e_{1}, e_{2}, \ldots, e_{n}\right]^{T}$ and $v=\left[v_{1}, v_{2}, \ldots, v_{n}\right]^{T}, E$ represent the energy stored in the system, and $P_{\text {diss }}$ represent the power dissipation of the system which is non-negative.

Definition 3.2 means that a passive $n$-port network only consumes energy and cannot generate energy for the system. The passivity definition leads to the wave scattering concept. The idea is that the total power flow is divided into two parts, the 
input power and output power. Detailed information about wave variable and wave scattering can be found in [8] and we will give some results directly.

For an $n$-port network, the scattering operator $S_{c}$ satisfies

$$
e-v=S_{c}(e+v)
$$

The scattering operator $S_{c}$ can be expressed in the frequency domain as a scattering matrix $S_{c}(s)$, where

$$
e(s)-v(s)=S_{c}(s)(e(s)+v(s))
$$

For two-port representation, this scattering matrix can be related to the hybrid matrix $h(s)$ in (3.4.1) as follows [3]:

$$
S_{c}(s)=\left[\begin{array}{cc}
1 & 0 \\
0 & -1
\end{array}\right](h(s)-I)(h(s)+I)^{-1}
$$

where $I$ is the identity matrix.

It is known from [3] that a two-port system is passive if and only if the $H_{\infty}$ norm of its scattering operator is less than or equal to one, i.e. $\left\|S_{c}(s)\right\|_{\infty} \leq 1$.

Note that, in other parts of this thesis, the force/position representation is used. However, for passivity analysis, force/velocity representation is usually chosen. This is because the power of a system can be directly calculated by the production of the force and velocity variables.

For passivity analysis of teleoperation systems, we usually consider the effort to be force and flow to be velocity. Then we have $e_{m}=F_{m}, e_{s}=F_{s}, v_{m}=V_{m}=\dot{X}_{m}$ and $v_{s}=V_{s}=\dot{X}_{s}$, where $F_{m}, F_{s}, X_{m}$ and $X_{s}$ were defined in section 2.1. The relationship 
between the hybrid matrix $H(s)$ of equation (2.1.1) and $h(s)$ of equation (3.4.1) can be derived,

$$
h(s)=\left[\begin{array}{cc}
s H_{11}(s) & H_{12}(s) \\
H_{21}(s) & s^{-1} H_{22}(s)
\end{array}\right]
$$

From [3], a system made up of passive elements is itself passive since the system will only consume energy and that a passive system can be realized by a system made up of passive elements.

\subsubsection{Robust Control Theory}

Most modern control designs are based on a mathematical model of the physical system. The relationship between models and reality is usually very complex. People can never find an ideal model having exactly the same responses as the real physical plant. Normally, the quality of a model depends on how closely the responses of the model match those of the real plant. A design technique must help make this gap as small as possible [15][44][50].

Systems that are able to tolerate certain plant variability and uncertainty are called robust. Because a model cannot be exactly the same as the real plant, uncertainty is an inevitable and important issue. The term "uncertainty" refers to the difference between models and reality and the mechanism used to describe these errors is called a representation of uncertainty. 


\section{Feedback Structure}

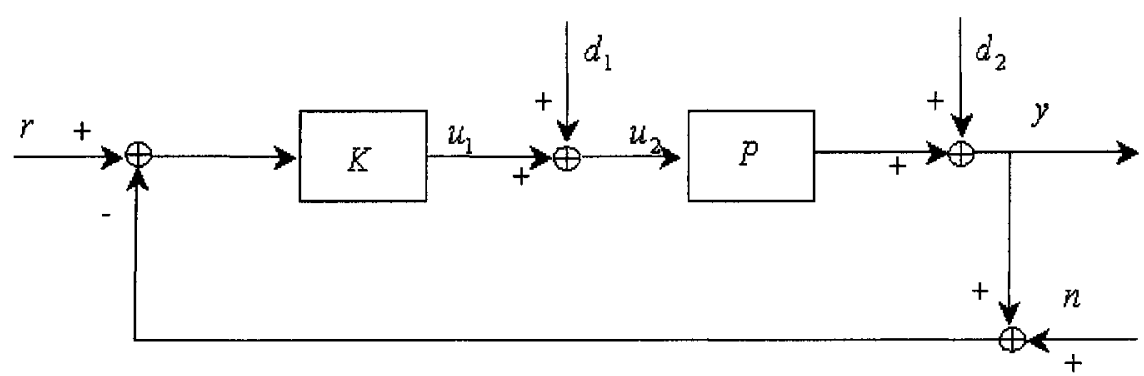

Figure 3.1: Standard feedback configurations.

Consider the standard feedback configuration shown in Figure 3.1, where $P$ represents the plant to be controlled; $K$ is the controller forced by command $r ; n$ is noise; $d_{1}$ and $d_{2}$ are the plant input disturbance and plant output disturbance respectively; system output is $y$. Here, all signals are assumed to be multivariable, and all transfer matrices are assumed to have multiple dimensions.

A designed system must be physically realizable. We first examine the conditions for the system shown in Figure 3.1 to be physically realizable.

\section{Definition 3.3}

A feedback system is said to be well-posed (physically realizable) if all closed-loop transfer matrices for the inputs, disturbances and noises are well-defined and proper.

Suppose that all the external signals $r, n, d_{1}$ and $d_{2}$ are specified and the closed-loop transfer matrices from these external signals to $u_{1}$ are well-defined and proper. According to robust theory, the output $y$ and all other signals are also 
well-defined and the related transfer matrices are proper. Furthermore, since the transfer matrices from $d_{2}$ and $n$ to $u_{1}$ are the same and differ from the transfer matrix from $r$ to $u_{1}$ by only a negative sign, the system is well-posed if and only if the transfer matrix from $\left[\begin{array}{l}d_{1} \\ d_{2}\end{array}\right]$ to $u=\left[\begin{array}{l}u_{1} \\ u_{2}\end{array}\right]$ exists and is proper.

We can now regroup the external input signals into the feedback loop as $w_{1}$ and $w_{2}$ and the input signals of the plant and the controller as $e_{1}$ and $e_{2}$. Then the feedback loop with the plant and the controller can be represented in Figure 3.2 and the system is well-posed if and only if the transfer matrix from $\left[\begin{array}{l}w_{1} \\ w_{2}\end{array}\right]$ to $e_{1}$ exists and is proper.

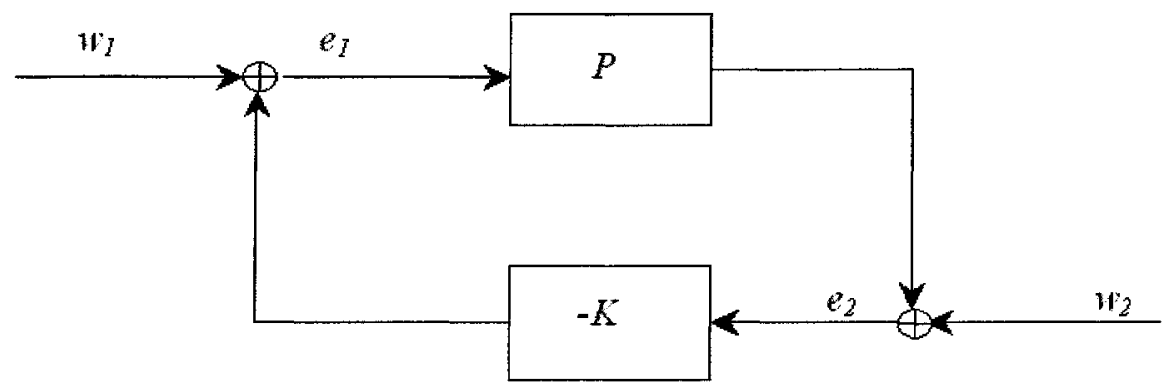

Figure 3.2: Internal stability analysis diagram.

It is shown that the feedback system in Figure 3.2 is well-posed if and only if $I-(-K(\infty)) P(\infty)$ is invertible [19][50].

The next problem is to give the internal stability definition. Internal stability is a basic requirement for a feedback system. It is because all interconnected systems may have some nonzero initial conditions and small errors, and in real application such errors 
may lead to unbounded signals at some other locations in the closed-loop system and bring some damage. Internal stability ensures that all signals in a system are bounded provided that the injected signals are bounded.

\section{Definition 3.4}

The system shown in Figure 3.2 is internally stable if the transfer matrix

$$
\left[\begin{array}{cc}
I & K \\
-P & I
\end{array}\right]^{-1}=\left[\begin{array}{cc}
I-K P & -K \cdot(I-P K) \\
P \cdot(I-K P) & I-P K
\end{array}\right]
$$

from $\left(w_{1}, w_{2}\right)$ to $\left(e_{1}, e_{2}\right)$ is in $R H_{\infty} . I$ is the identity matrix.

So, to determine whether a system is internally stable, we only need to test whether each of the four transfer matrices in the above is in the $R H_{\infty}$ space.

\section{Model Uncertainty}

As mentioned previously, the control design technique must minimize the difference between the model and real plant. A good model should be simple so that the design method can be easily applied and, at the same time, complex enough to ensure that designs based on the model are valid and the required performance can be satisfied.

The quality of a model largely depends on our knowledge of the physical mechanisms that cause differences between the model and the real plant as well as our capability to represent the mechanisms.

A common technique to model the plant is to assign the plant to a set. The set can be either structured or unstructured, where unstructured uncertainty considers the neighborhood of the model and is adopted in this thesis. 


\section{Robust Control Theorem}

The robust stability criterion applied for multiplicative unstructured uncertainties is given in [50]. For the following discussion, we assume the system model is described by the following set $\Pi$ of models, in multiplicative perturbed form

$$
\Pi=\left\{[I+W(s) \Delta(s)] \cdot P_{0}(s)\right\}
$$

where $W(s), \Delta(s) \in R H_{\infty} \quad$ and $\|\Delta(s)\|_{\infty}<1 ; P_{0}(s) \quad$ is the nominal model. $P(s)=[I+W(s) \Delta(s)] \cdot P_{0}(s)$ is a real model. $W(s)$ characterizes the spatial and frequency structure of the uncertainty and it restricts $P(s)$ to a neighbourhood of the nominal model $P_{0}(s)$.

Figure 3.3 shows the relationship between nominal model $P_{0}(s)$, real model $P(s)$ and the uncertainty range $W(s)$. Figure 3.4 shows the structure of an output multiplicative perturbed system.

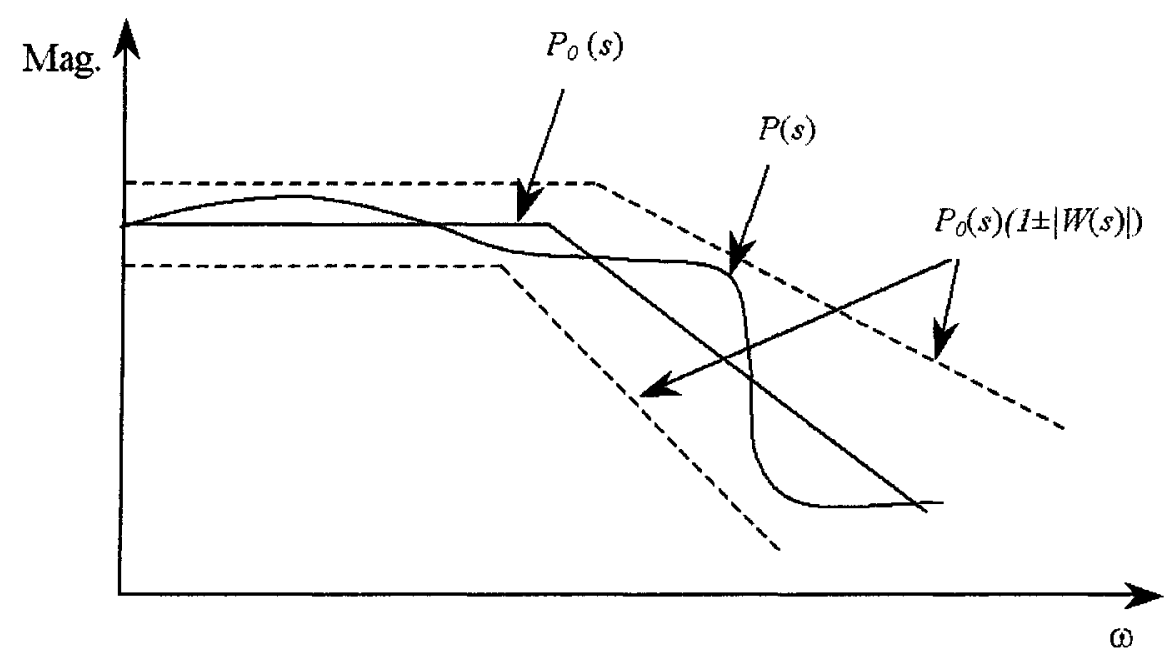

Figure 3.3: Typical behaviour of multiplicative uncertainty. 


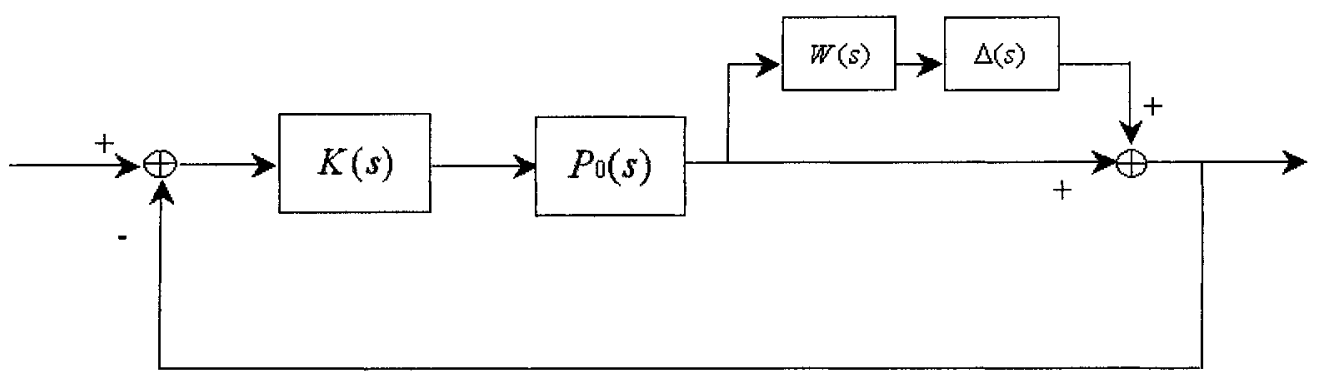

Figure 3.4: Output multiplicative perturbed systems.

Before we proceed, we first introduce the complementation matrix $T$ for the system, which is defined as:

$$
T(s)=I-S(s)
$$

where $S(s)=\left[I+P_{0}(s) K(s)\right]^{-1}$ and $I$ is the identity matrix.

\section{Theorem 3.1 (Robust stability condition):}

Let $\Pi=\left\{(I+W(s) \Delta(s)) P_{0}(s): W(s), \Delta(s) \in R H_{\infty}\right\}$ and let $K(s)$ be a stabilizing controller for the nominal plant $P_{0}(s)$. Then the closed-loop system shown in Figure 3.4 is well-posed and internally stable for all $\Delta \in R H_{\infty}$ with $\|\Delta(s)\|_{\infty}<1$ if and only if $\|T(s) W(s)\|_{\infty} \leq 1$

It is helpful to give an explanation for the uncertainty functions $W(s)$ and $\Delta(s)$. From Figure 3.3, for SISO (single input single output) systems, $W(s)$ represents the maximum deviation from the nominal function so that the two dashed line functions can be represented by $P_{0}(s) \cdot(I \pm|W(s)|)$. Since $|\Delta(s)|<1$, we know the real plant $P(s)=P_{0}(s) \cdot(I+W(s) \Delta(s))$ must lie between the two dashed lines. This is why we call 
$W(s)$ a bound for the uncertainty.

\subsubsection{Robust Stability Constraint for SISO Systems}

In this thesis, the uncertainty of the remote environment is considered as the main source of modeling error. Once the range of the environment impedance uncertainty can be determined, a constraint will be posed on the controller parameters that can stabilize the system. Next, the robust stability design for SISO systems is discussed and the case study of the design will be shown in chapter 4 .

As shown in equation (2.2.25), for a SISO teleoperation system, the open loop gain can be calculated from its hybrid representation as follows.

$$
G(s)=\frac{-H_{12}(s) H_{21}(s) Z_{e}(s)}{\left(H_{11}(s)+Z_{h}(s)\right)\left(1+H_{22}(s) Z_{e}(s)\right)}
$$

where $Z_{h}(s)$ and $Z_{e}(s)$ are the human operator and environment impedances respectively. $H_{11}(s), H_{12}(s), H_{21}(s)$ and $H_{22}(s)$ are the elements of the two-port hybrid matrix.

In [7], an analysis for the stability problem with the variation of environment and human operator impedance was proposed. Here we only consider the uncertainty of the remote environment.

The SISO closed-loop system to be studied is modeled in Figure 3.5. The entire teleoperation system including the MSN, human operator and the environment are combined into one closed loop system. 


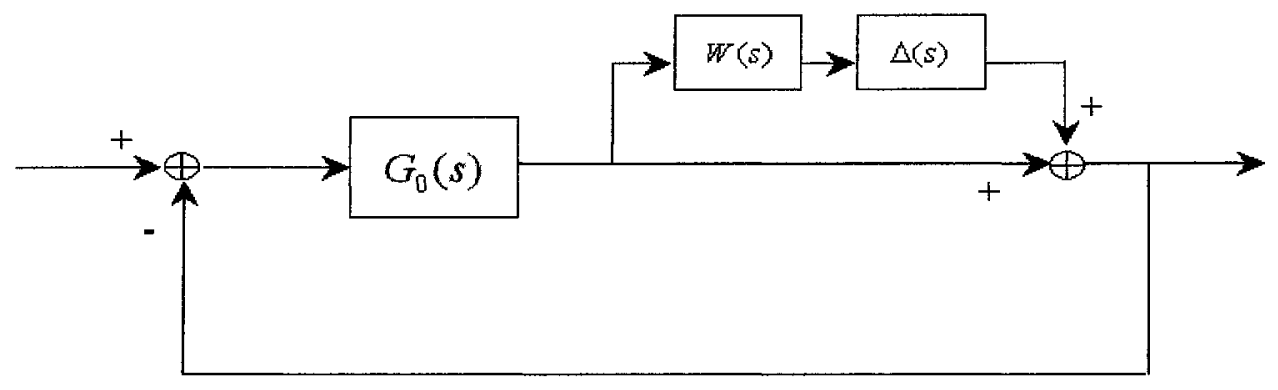

Figure 3.5: Closed-loop system to be studied.

The open loop transfer function of the system is $G(s)=G_{0}(s)(1+W(s) \Delta(s))$, where $G_{0}(s)$ is the nominal open loop transfer function and $W(s) \Delta(s)$ represents the uncertainty.

Comparing the model of Figure 3.4 and Figure 3.5, we have

$$
G_{0}(s)=K(s) P_{0}(s)
$$

Assume the nominal environmental impedance is $Z_{e 0}(s)=m_{e 0} s^{2}+b_{e 0} s+k_{e 0}$, we can derive the nominal open loop gain from the hybrid parameters according to equation

$$
G_{0}(s)=\frac{-H_{12}(s) H_{21}(s) Z_{e 0}(s)}{\left(H_{11}(s)+Z_{h}(s)\right)\left(1+H_{22}(s) Z_{e 0}(s)\right)}
$$

For the real model, $Z_{e}(s)$ is the real value of the environment impedance. We have

$$
\begin{aligned}
G(s) & =G_{0}(s) \cdot(1+W(s) \Delta(s)) \\
& =\frac{-H_{12}(s) H_{21}(s) Z_{e}(s)}{\left(H_{11}(s)+Z_{h}(s)\right)\left(1+H_{22}(s) Z_{e}(s)\right)}
\end{aligned}
$$




$$
\begin{aligned}
& =\left(\frac{-H_{12}(s) H_{21}(s)}{H_{11}(s)+Z_{h}(s)} \cdot \frac{Z_{e 0}(s)}{1+H_{22}(s) Z_{e 0}(s)}\right) \cdot\left(\frac{1+H_{22}(s) Z_{e 0}(s)}{Z_{e 0}(s)} \cdot \frac{Z_{e}(s)}{1+H_{22}(s) Z_{e}(s)}\right) \\
& =G_{0}(s) \cdot\left[\frac{1+H_{22}(s) Z_{e 0}(s)}{Z_{e 0}(s)} \cdot \frac{Z_{e}(s)}{1+H_{22}(s) Z_{e}(s)}\right]
\end{aligned}
$$

From equation (3.4.11), the multiplicative uncertainty of the system is

$$
\begin{aligned}
W(s) \Delta(s) & =\frac{1+H_{22}(s) Z_{e 0}(s)}{Z_{e 0}(s)} \cdot \frac{Z_{e}(s)}{1+H_{22}(s) Z_{e}(s)}-1 \\
& =\frac{Z_{e}(s)-Z_{e 0}(s)}{Z_{e 0}(s)} \cdot \frac{1}{1+H_{22}(s) Z_{e}(s)}
\end{aligned}
$$

Also, from equation (3.4.8), we have the complementation matrix $T(s)$

$$
T(s)=1-S(s)=\frac{G_{0}(s)}{1+G_{0}(s)}
$$

where $G_{0}(s)$ is given by equation (3.4.10).

From equation (3.4.12) and the physical model introduced in section 2.2 , we can find out the bounding transfer function $W(s)$ by scanning a prescribed environment impedance uncertainty range while all the other physical model parameters are known.

According to Theorem 3.1, if the system satisfies,

$$
\|T(s) W(s)\|_{\infty} \leq 1
$$

it is internally stable. For the two teleoperator structures introduced in subsection 2.2 .2 , we know the hybrid matrix elements for position-force and modified position-force structures in equation (2.2.17) and (2.2.22). We can first find out $T(s)$ and $W(s)$. Then 
the stability constraint of equation (3.4.14) can be achieved.

It should be noted that the above multiplicative uncertainty is a function of environment impedance and controller parameters. Usually, the upper bound is a function of the controller parameters. However, such a function is often very difficult to achieve. As an alternate way, we can first divide the controller parameters into many segments and find the bounding function of each segment; then we can find the parameter range that stabilizes the system for each segment; finally, combining the stability analysis results of all these segments, the stability constraint of the system can be achieved. 


\section{Chapter 4}

\section{Case Studies}

In the previous chapters, the robust controller design approach for bilateral teleoperators was introduced. To validate the proposed method and show the details of the proposed design procedure, two case studies were performed by simulation using $\mathrm{Matlab}^{\circledR}$ software.

For the case studies, the physical model parameters were deliberately chosen in order to represent the typical teleoperation system and environment in telesurgery applications. The selection of these parameters mainly depends on the physical models parameters used in the simulations and experiments of [7][23][30][33][48].

In order to examine the effectiveness of the proposed method, simulation results based on the passivity theory is presented as well. The results of passivity based design and the proposed robust design are compared in the case studies. 


\subsection{Design Flow}

Readers may have been familiar with the new design method after reading the previous chapters. However, they may still wonder how to start the design and what the complete design flow is like. A flowchart describing the steps of the design process is shown in Figure 4.1.

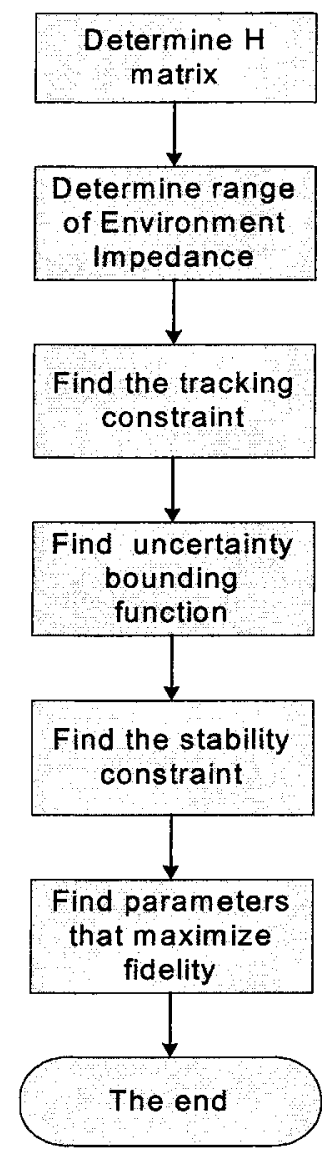

Figure 4.1: Proposed design flow.

For the proposed design procedure, the first step is to find the hybrid matrix for the teleoperator; in the second step, we need to determine the environment uncertainty range according to our prior knowledge or experiments; then the parameter range that satisfies 
the free space tracking constraint is calculated; next, the parameter range that stabilizes the overall system subject to the environment uncertainty is found; then, the range of parameters that both stabilize the system and satisfies the tracking constraint is found; finally, the parameter that maximizes the fidelity measure will be chosen from the above range and the optimization process is completed.

\subsection{Position-Force Controlled Teleoperator (Case 1)}

In chapter 2 , the dynamics of the position-force controlled structure were analyzed and the two-port hybrid matrix was derived. In the following sections, the notations will be followed.

First we choose the values of the physical model parameters. The inertia, damping and stiffness of human operator are $m_{h}=1.5 \mathrm{~kg}, \quad b_{h}=1.0 \mathrm{Ns} / \mathrm{m}$ and $k_{h}=8 \mathrm{~N} / \mathrm{m}$, respectively; the master and slave robots' inertias are set to be the same, with $m_{m}=m_{s}=0.5 \mathrm{~kg}$; the slave's damping ratio is $b_{s}=6 \mathrm{Ns} / \mathrm{m}$; the stiffness of master and slave are $k_{m}=k_{s}=0.5 \mathrm{~N} / \mathrm{m}$; the forward position gain of the teleoperator is $g=300 \mathrm{~N} / \mathrm{m}$; the nominal environment impedance that the slave is contacting with is

considered to be a pure stiffness of $k_{e 0}=150 \mathrm{~N} / \mathrm{m}$, i.e. $Z_{e 0}=k_{e 0}=150 \mathrm{~N} / \mathrm{m}$; the environment uncertainty range is $Z_{e}=k_{e} \in[50,300] \mathrm{N} / \mathrm{m}$. The above environment setting corresponds to a typical soft environment in surgical operations.

For the position-force teleoperator, the damping ratio of the master robot $b_{m}$ plays a key part in the overall system. In general, improving the damping of the master 
manipulator tends to stabilize the system and, at the same time, degrades the system performance since the human operator must use excessive effort to operate the system. On the contrary, decreasing the damping of the master will improve the performance however the system is less likely to be stable [10][33]. In this case study, the master damping $b_{m}$ is the only controller parameter, which can be chosen from a range of $[5,30] N s / m$.

\subsubsection{Tracking Constraint}

In section 3.3 , free space tracking is considered to be a constraint for the optimization problem. The transfer function for tracking error is the sensitivity function, which is given by equation (3.3.3), that is

$$
S_{e n}(s)=1+H_{21}(s)
$$

From equation (2.2.17), we have

$$
H_{21}(s)=-\frac{g}{m_{s} s^{2}+b_{s} s+k_{s}+g} .
$$

Then the sensitivity function can be written as:

$$
S_{e n}(s)=1+H_{21}(s)=\frac{m_{s} s^{2}+b_{s} s+k_{s}}{m_{s} s^{2}+b_{s} s+k_{s}+g}
$$

The input signal for $S_{e n}(s)$ is the master manipulator position $X_{m}(s)$ and the output is the position error between the master and slave manipulators, i.e. $X_{m}(s)+X_{s}(s)$.

Next, suppose that the free space tracking requirement is: the error between the master and slave manipulators is less than $10 \%$ of the input signal $X_{m}$, where $X_{m}$ is a 
unit sinusoid signal with frequency lower than $0.5 \mathrm{~Hz}$.

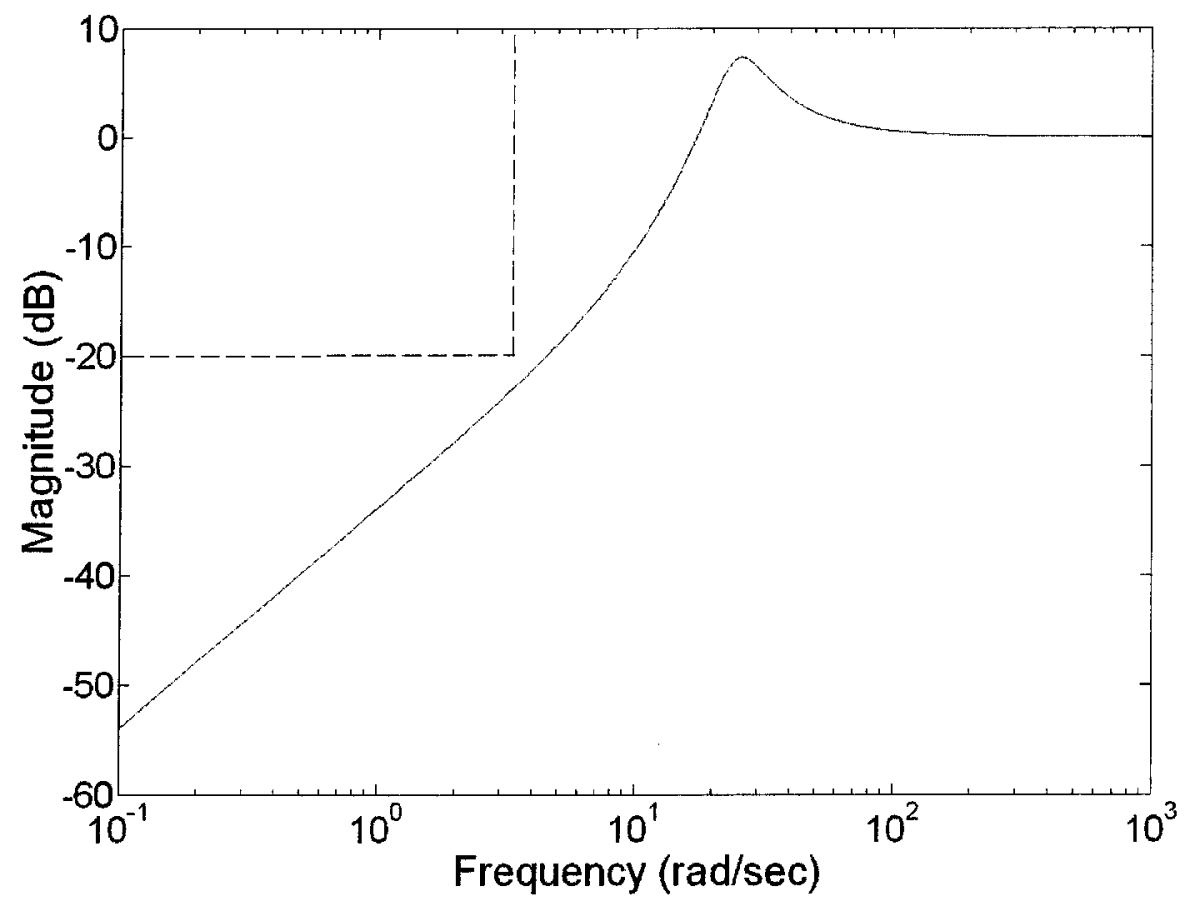

Figure 4.2: Tracking performance constraint (Case 1).

Since all the parameters of equation (4.2.1) are known and fixed, we only need to prove that the above tracking requirement is satisfied by the current system. The Bode magnitude plot for the transfer function $S_{e n}(s)$ is shown in Figure 4.2. Since $10 \%=0.1=-20 \mathrm{~dB}$, the dashed lines specify the ceiling for the tracking error. Because the frequency response of $S_{e n}(s)$ is below the dashed lines over the range of frequencies considered, we can say that the free-space tracking requirement is satisfied. 


\subsubsection{Robust Stability Analysis}

\section{Stability of Nominal System}

According to Theorem 3.1, we first need to check the system stability in the nominal environment. The nominal open loop gain of the system, $G_{0}(s)$, can be obtained by equation (3.4.10), that is

$$
G_{0}(s)=\frac{-H_{12}(s) H_{21}(s) Z_{e 0}(s)}{\left(H_{11}(s)+Z_{h}(s)\right)\left(1+H_{22}(s) Z_{e 0}(s)\right)}
$$

According to Figure 3.5, without considering the uncertainty term, the closed-loop transfer function can be obtained

$$
\hat{G}(s)=\frac{G_{0}(s)}{1+G_{0}(s)}
$$

From the plot of poles of the transfer function $\hat{G}(s)$, we can determine the stability of the closed-loop system. If all the poles are located in the left-half plane, the system is stable.

According to equations (3.4.10) and (2.2.17), we know that $\hat{G}(s)$ is a function of the controller parameter $b_{m}$. Figure 4.3 shows the plot of poles of $\hat{G}(s)$ for $b_{m} \in[5,30] N s / m$. It is seen from the figure that all the closed-loop poles of the nominal plant are located in the left half of the complex plane. So we know that the nominal closed loop system is always stable for the controller parameter $b_{m}$ in its prescribed range $b_{m} \in[5,30] N s / m$. 


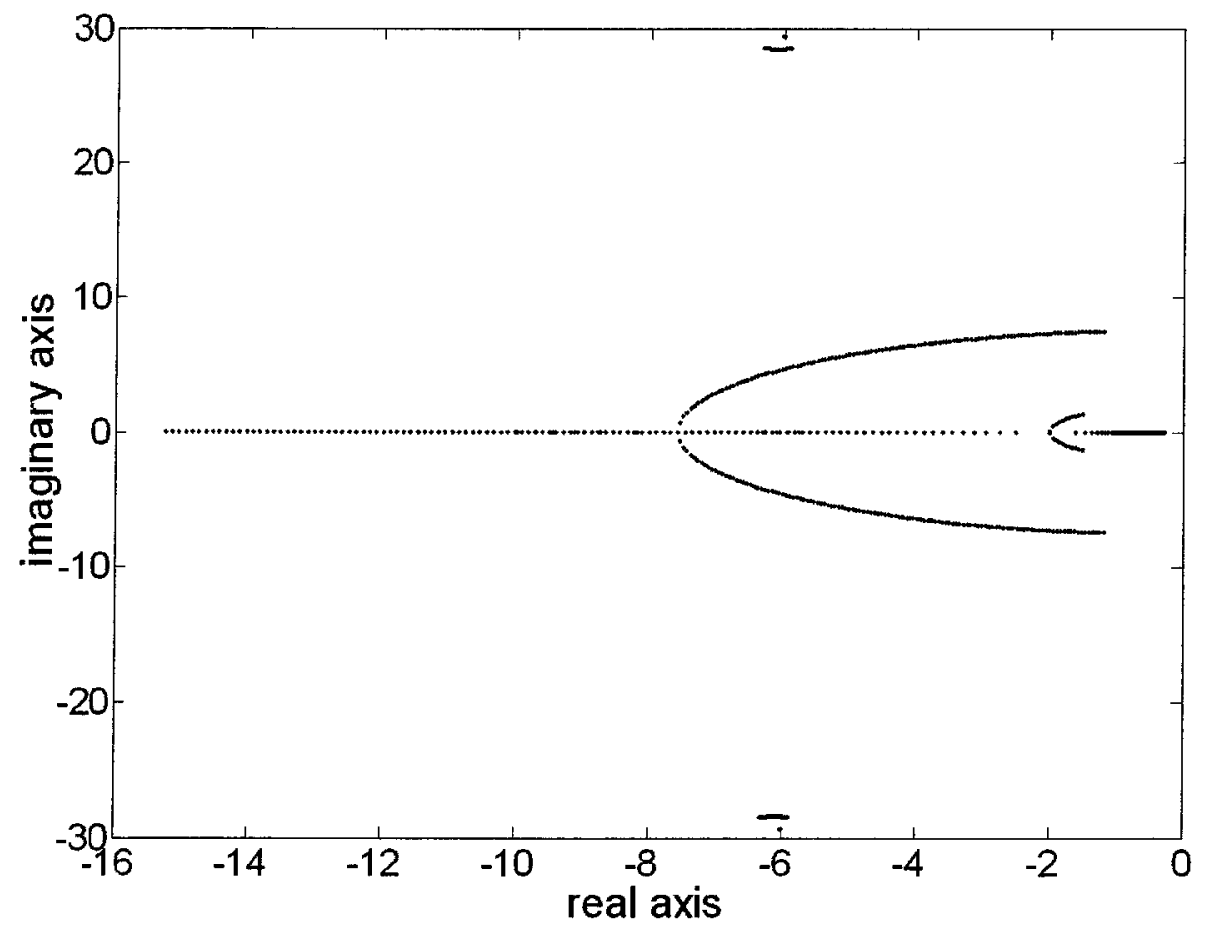

Figure 4.3: Nominal closed-loop system poles (Case 1).

\section{Finding the Bounding Function}

The next step of the robust stability analysis is to find the uncertainty bounding function $W(s)$ described in subsection 3.4.2. Let's first look at the uncertainty function defined in equation (3.4.12). For convenience, we rewrite the equation as:

$$
W_{d}(s)=W(s) \Delta(s)=\frac{Z_{e}(s)-Z_{e 0}(s)}{Z_{e 0}(s)} \cdot \frac{1}{1+H_{22}(s) Z_{e}(s)}
$$

From equation (3.4.12), it is observed that this uncertainty function only contains the fourth element $H_{22}(s)$ of the hybrid matrix. From equation (2.2.17), for the position-force controlled teleoperator, we know that 


$$
H_{22}(s)=\frac{1}{Z_{s}(s)+g}=\frac{1}{m_{s} s^{2}+b_{s} s+k_{s}+g}
$$

It is clear that the uncertainty term $W_{d}(s)=W(s) \Delta(s)$ is not a function of the controller parameter $b_{m}$. As a result, finding the bounding function is relatively easy.

By systematically varying the environment impedance $Z_{e}$ in its prescribed uncertainty range $Z_{e}=k_{e} \in[50,300] \mathrm{N} / \mathrm{m}$, the set of uncertainty transfer functions $W_{d}(s)=W(s) \Delta(s)$ are shown in Figure 4.4.

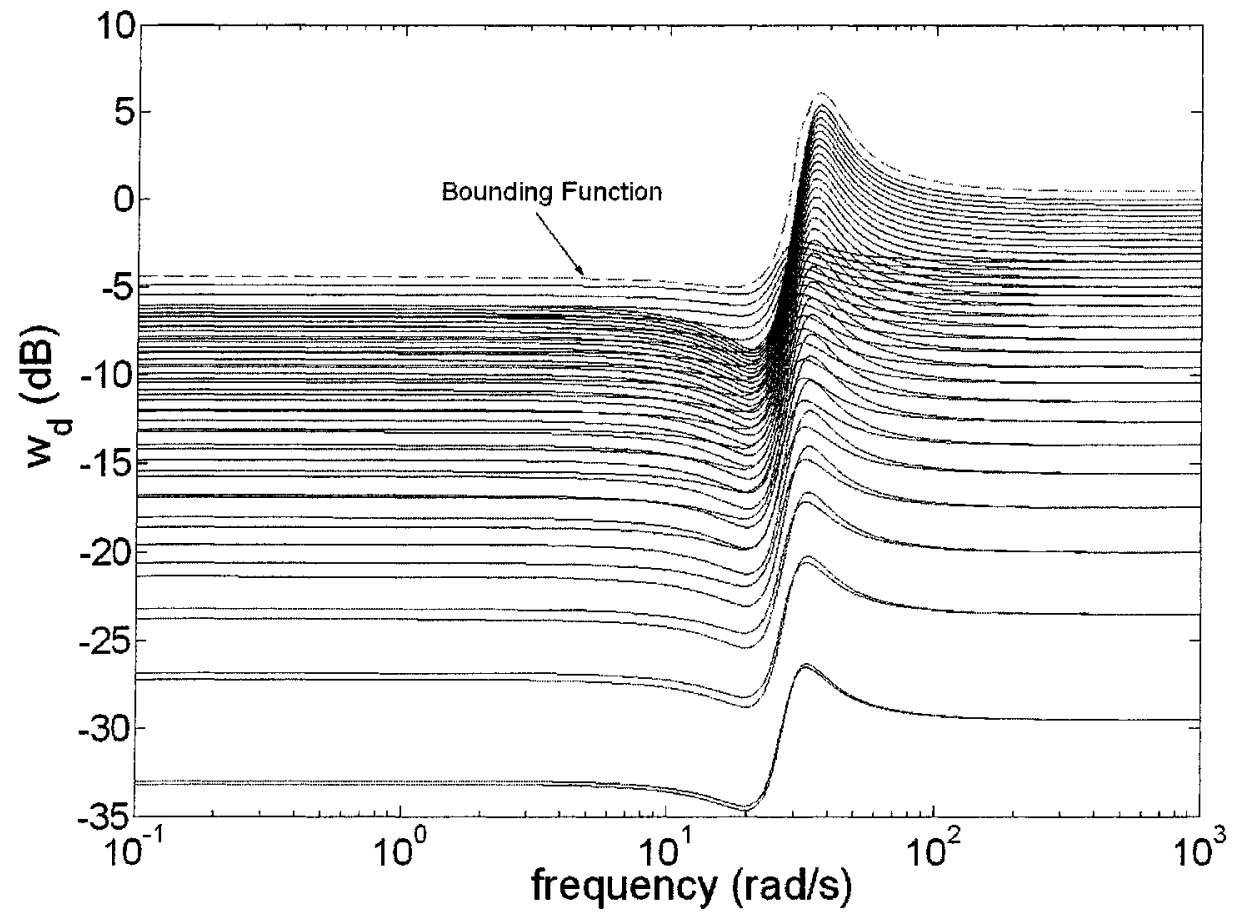

Figure 4.4: Find the bounding function (Case 1).

In Figure 4.4, each solid curve corresponds to a particular environment impedance in the uncertainty range $Z_{e}=k_{e} \in[50,300] N / m$, the dashed curve above the top of all the 
solid curves is the bounding function $W(s)$ which is given by,

$$
W(s)=\frac{1.069 s^{4}+27.44 s^{3}+1889 s^{2}+24030 s+731600}{s^{4}+19.43 s^{3}+2295 s^{2}+19770 s+1204000}
$$

For very simple systems, the bounding function can be achieved analytically. However, in most cases, this bounding function can be only obtained numerically. In the two case studies, the bounding functions are achieved using the Robust Control Toolbox of Matlab ${ }^{\circledR}$ software.

\section{Internal Stability Analysis}

So far we have found the bounding function $W(s)$ for the system. Then the controller parameters that guarantee internal stability of the system can be determined. From Theorem 3.1, we know that if $\|T(s) W(s)\|_{\infty} \leq 1$, the internal stability of the system can be guaranteed. Next, we show how to find out the complementation matrix $T(s)$.

Rewriting equations (3.4.13) and (3.4.10), we have

$$
T(s)=1-S(s)=\frac{G_{0}(s)}{1+G_{0}(s)}
$$

with

$$
G_{0}(s)=\frac{-H_{12}(s) H_{21}(s) Z_{e 0}(s)}{\left(H_{11}(s)+Z_{h}(s)\right)\left(1+H_{22}(s) Z_{e 0}(s)\right)}
$$

The parameters $H_{11}(s), H_{12}(s), H_{21}(s)$, and $H_{22}(s)$ can be calculated according to equation (2.2.17).

The internal stability function $\|T(s) W(s)\|_{\infty}$ is shown as a function of the master 
damping $b_{m}$ in Figure 4.5.

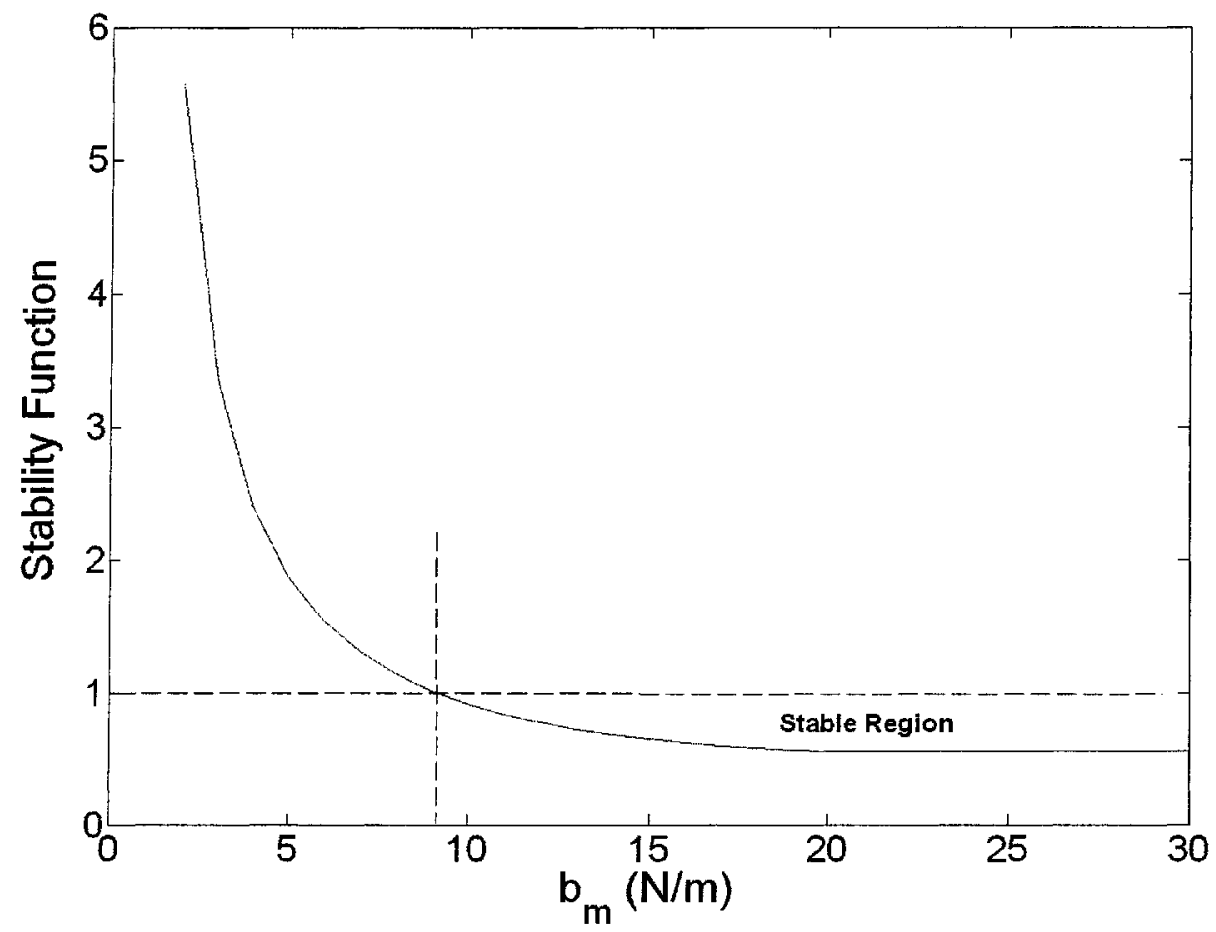

Figure 4.5: Internal stability function (Case 1).

In Figure 4.5, the stability function value determines the internal stability of the system. It was found that if the controller parameter $b_{m} \geq 9.5$, the system is internally stable. In other words, the stabilizing controller parameter range is $b_{m} \in[9.5,30] \mathrm{Ns} / \mathrm{m}$, which is a subset of $b_{m} \in[5,30] N s / m$.

\subsubsection{Fidelity Evaluation}

Now, the range of parameter $b_{m}$ that satisfies both stability and tracking constraints has already been found. The next design step is to calculate the controller parameter value 
that maximizes the system fidelity within the range, i.e., parameter $b_{m}$ that minimizes the fidelity evaluation function value $D_{z}$ with $b_{m} \in[5,30] N_{s} / m$. From equation (3.2.3), the nominal impedance transmitted to the human operator for a SISO system is

$$
Z_{t}(s)=\frac{F_{m}(s)}{X_{m}(s)}=H_{11}(s)-\frac{H_{12}(s) H_{21}(s) Z_{e 0}(s)}{1+H_{22}(s) Z_{e 0}(s)}
$$

From equation (3.2.3) and (2.2.17), it is observed that $Z_{t}$ is a function of controller parameter $b_{m}$. Thus we can find the value for controller parameter $b_{m}$ that minimizes the fidelity measure function $D_{z}\left(Z_{e 0}\right)=\left\|\left(Z_{t}(s)-Z_{e 0}(s)\right) \cdot l(s)\right\|_{\infty}$ for the nominal environment impedance. Here, $l(s)$ is a low-pass filter with a bandwidth of $2 \mathrm{~Hz}$. An appropriate filter is

$$
l(s)=\frac{143900}{s^{4}+62.33 s^{3}+1730 s^{2}+23650 s+143900}
$$

The Bode diagram for $l(s)$ is shown in Figure 4.6.

Since we have already known $Z_{t}(s), l(s)$ and $Z_{e 0}(s)$. The relationship between fidelity function value $D_{z}\left(Z_{e 0}\right)$ and the controller parameter $b_{m}$ is depicted in Figure 4.7. It is clear that smaller damping ratio $b_{m}$ corresponds to smaller fidelity function value and better fidelity.

Considering both the stability and tracking constraint, we can find that the controller parameter that minimizes the fidelity function is $b_{m}=9.5$, which corresponds to the fidelity function value of $D_{z}=71$. So far the robust design is completed. 


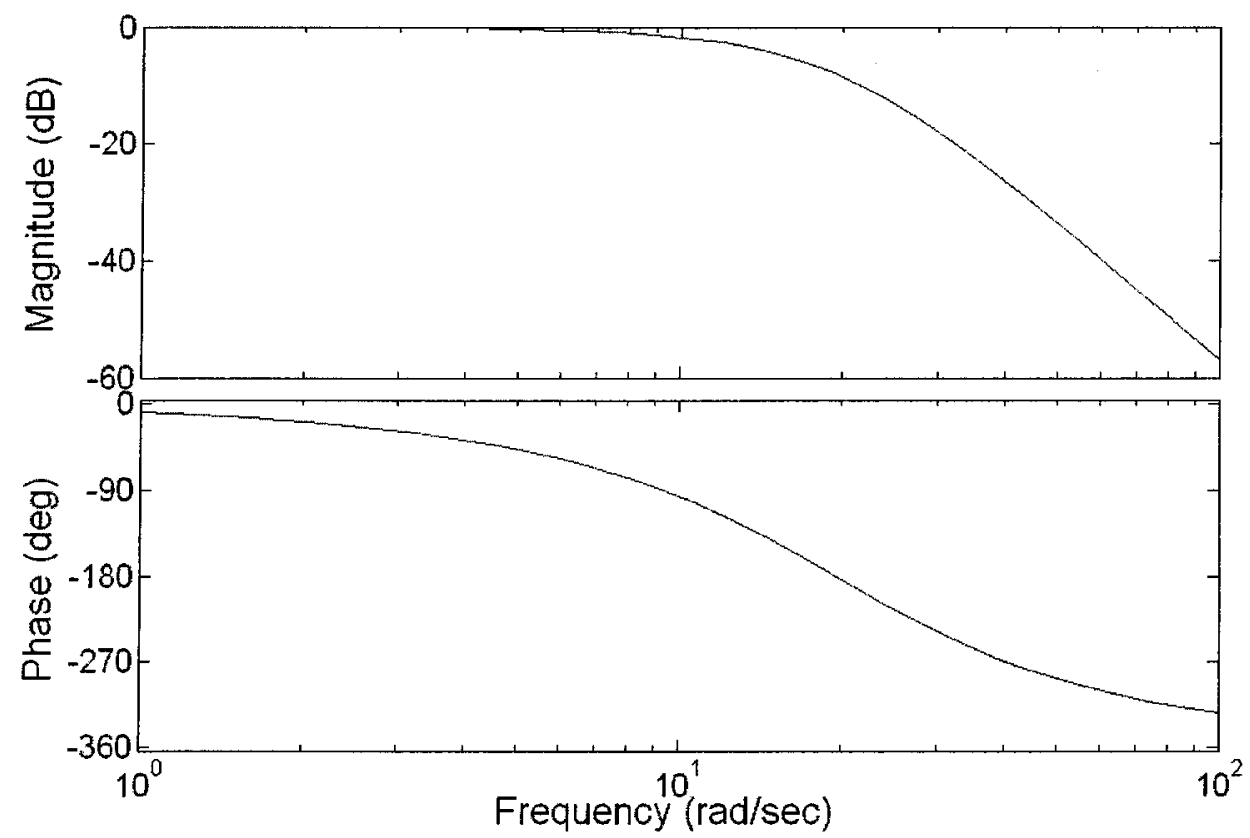

Figure 4.6: Bode diagram of $l(s)$.

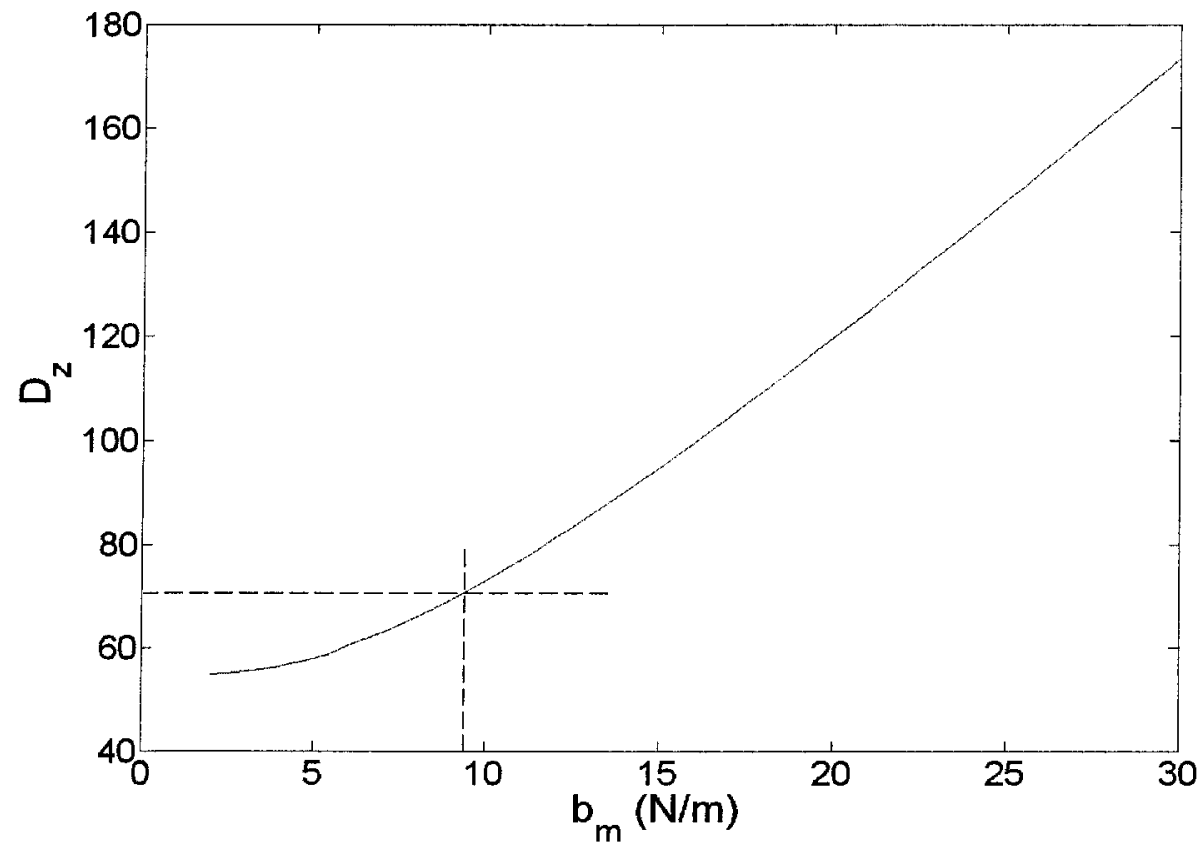

Figure 4.7: Fidelity function (Case 1). 


\subsubsection{Design Based on Passivity Theorem}

According to the passivity theory shown in subsection 3.4 .1 , the infinity norm of the scattering matrix $\left\|S_{c}(s)\right\|_{\infty}$ can be used to determine the passivity of the system. Rewrite the scattering matrix defined in equation (3.4.5).

$$
S_{c}(s)=\left[\begin{array}{cc}
1 & 0 \\
0 & -1
\end{array}\right](h(s)-I)(h(s)+I)^{-1}
$$

Since we have already known how to calculate the matrix $h(s)$ in equation (3.4.6), we can determine whether the system is passive according to what we introduced in section 3.4.1. The principle is that if $\left\|S_{c}\right\|_{\infty}$ is smaller or equal to 1 , the system is passive.

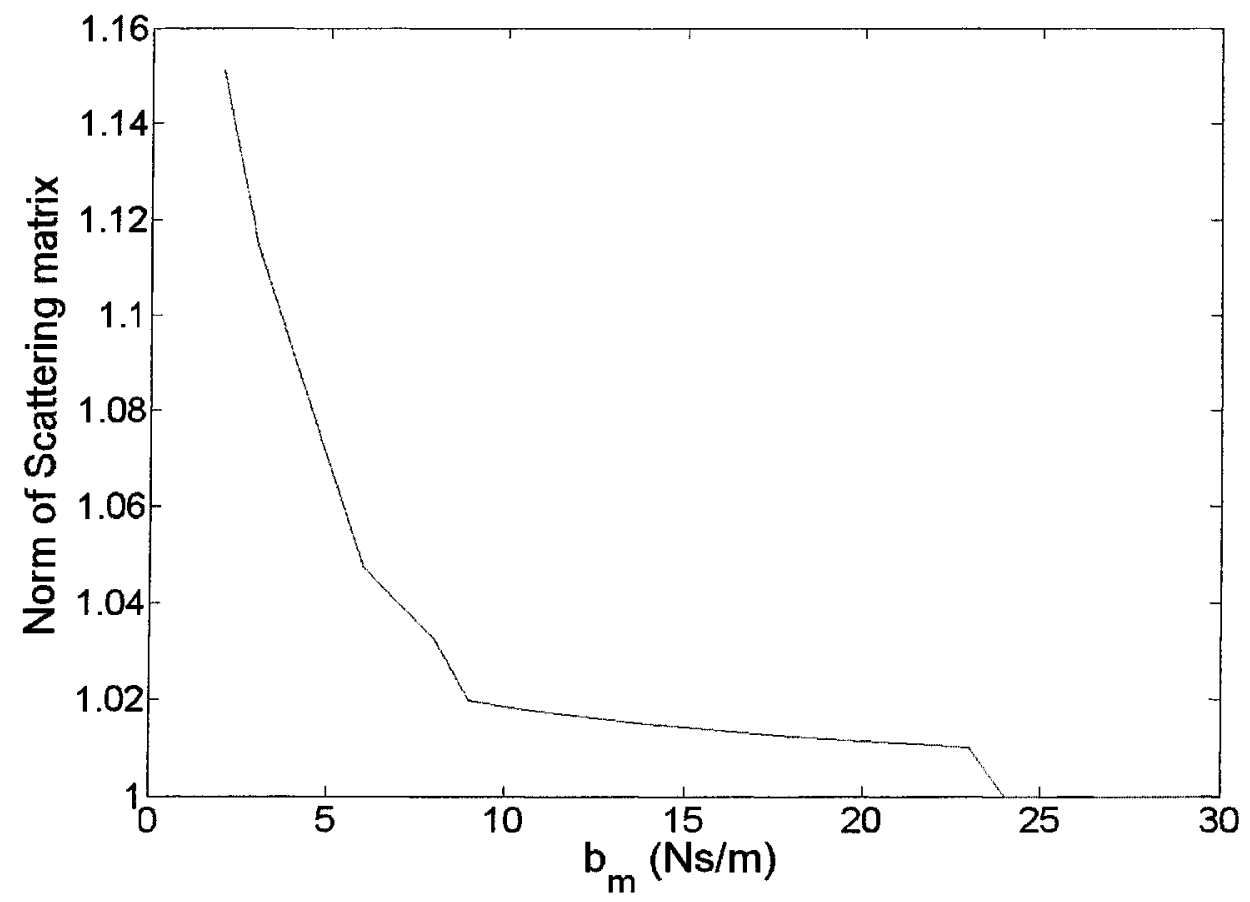

Figure 4.8: Passivity analysis (Case 1). 
The infinity norm of the scattering matrix, $\|S(s)\|_{\infty}$, is shown as a function of controller parameter $b_{m}$ in Figure 4.8. It is found that the system is passive only when $b_{m} \geq 24 N s / m$. In other words, $b_{m}$ must be larger or equal to $24 N s / m$ for the system to be stable according to the passivity theory. From the previous Figure 4.7, the best fidelity function value is $D_{z}=138$. This value is much larger than the one obtained based on the robust design, i.e. $D_{z}=71$. In other words, better fidelity is achieved when robust design approach is used.

\subsubsection{Fidelity Comparison}

Based on the previous analysis, the design results can be summarized: for robust control design, the best fidelity can be achieved when the controller parameter $b_{m}=9.5$ and, for passivity design, the best fidelity is achieved when $b_{m}=24$. The difference of the fidelity function $D_{z}$ has already shown that the robust design approach can achieve better fidelity than the passive design. However, such a comparison is not very straightforward and the validity of the fidelity function still needs to be proven. So we will give a direct comparison of the fidelity of the two design methods.

As mentioned in section 2.1 , the fidelity comparison can be conducted by showing all four force and position responses of both the master and slave manipulators. The position and force transfer functions have already been derived in equations (2.1.7) (2.1.8) (2.1.10) and (2.1.11). These responses are functions of the controller parameter $b_{m}$.

The position and force responses are shown in Figure 4.9. In the figure, the position 
and force error magnitudes and phases are calculated and compared at different frequencies. The position and force errors are relative error between the master and slave, i.e. $\operatorname{err} x=\left(x_{m}+x_{s}\right) / x_{m}$ and $\operatorname{errf}=\left(f_{m}-f_{s}\right) / f_{m}$. The input signal is $f_{h}=1-\cos \omega t$. The input signal frequencies are chosen to be $0.4 \mathrm{~Hz}, 0.8 \mathrm{~Hz}, 1.2 \mathrm{~Hz}$, and $1.6 \mathrm{~Hz}$. The robust and passive situations correspond to $b_{m}=9.5$ and $b_{m}=24$ respectively.

From Figure 4.9, it can be seen that the position errors are exactly the same for both robust and passive situations for different frequencies. This is because the position error between master and slave manipulators for this position-force based teleoperator is not a function of the controller parameter $b_{m}$. This can be proven by the transfer functions of $X_{m}(s)$ and $X_{s}(s)$. As a result, we can just compare the force feedback fidelity of these two design methods.

It was observed that for all four input frequencies, the force tracking error between the master and slave of robust design are much smaller than that of passive design. For example, for input frequency of $0.8 \mathrm{~Hz}$, the magnitude error is 0.05 for robust design and 0.28 for passive design and the angle errors are $29^{\circ}$ and $55^{\circ}$ respectively. The results show that the fidelity is greatly improved when the robust design method is applied. 

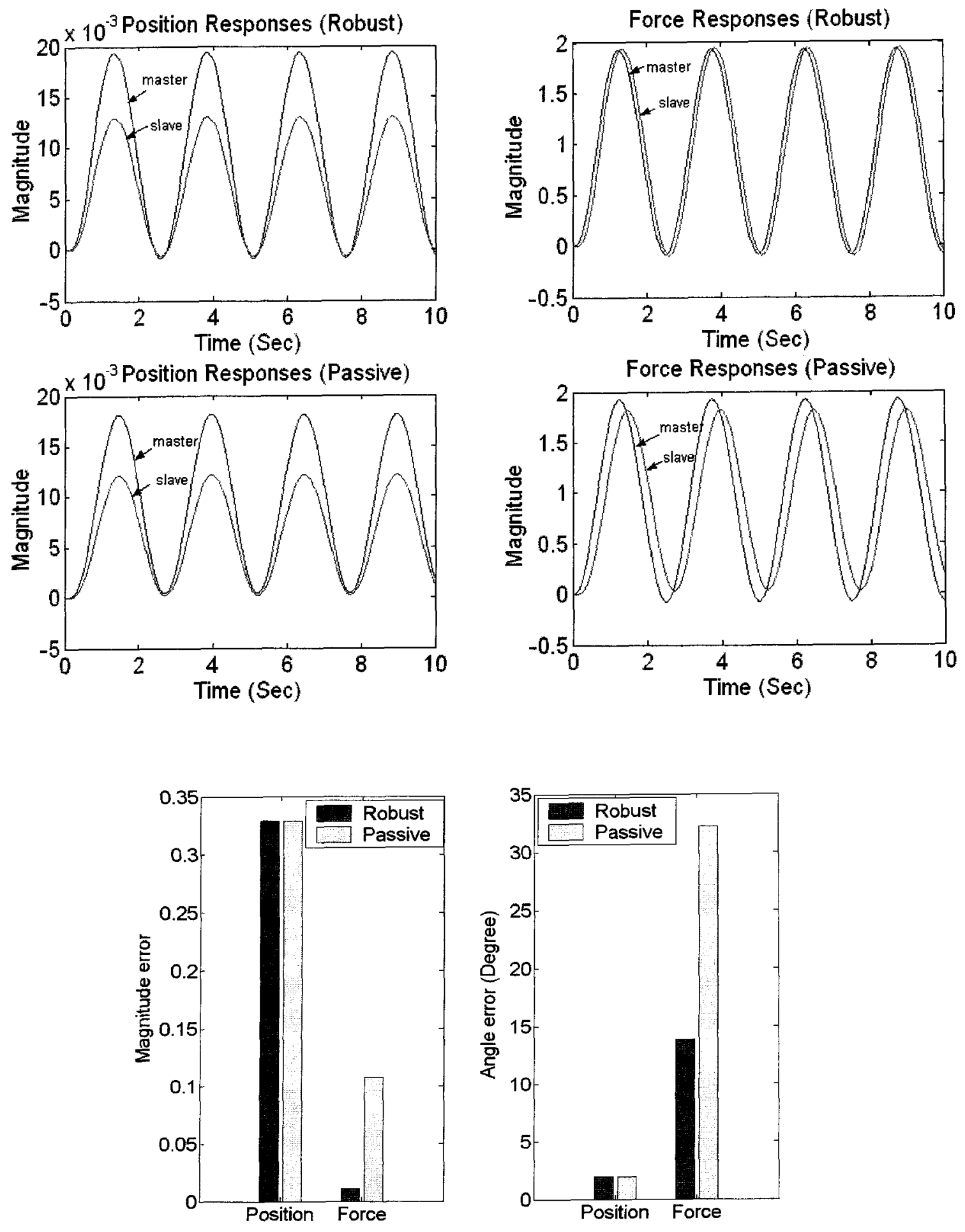

Figure 4.9 (a): Frequency $=0.4 \mathrm{~Hz}$. 

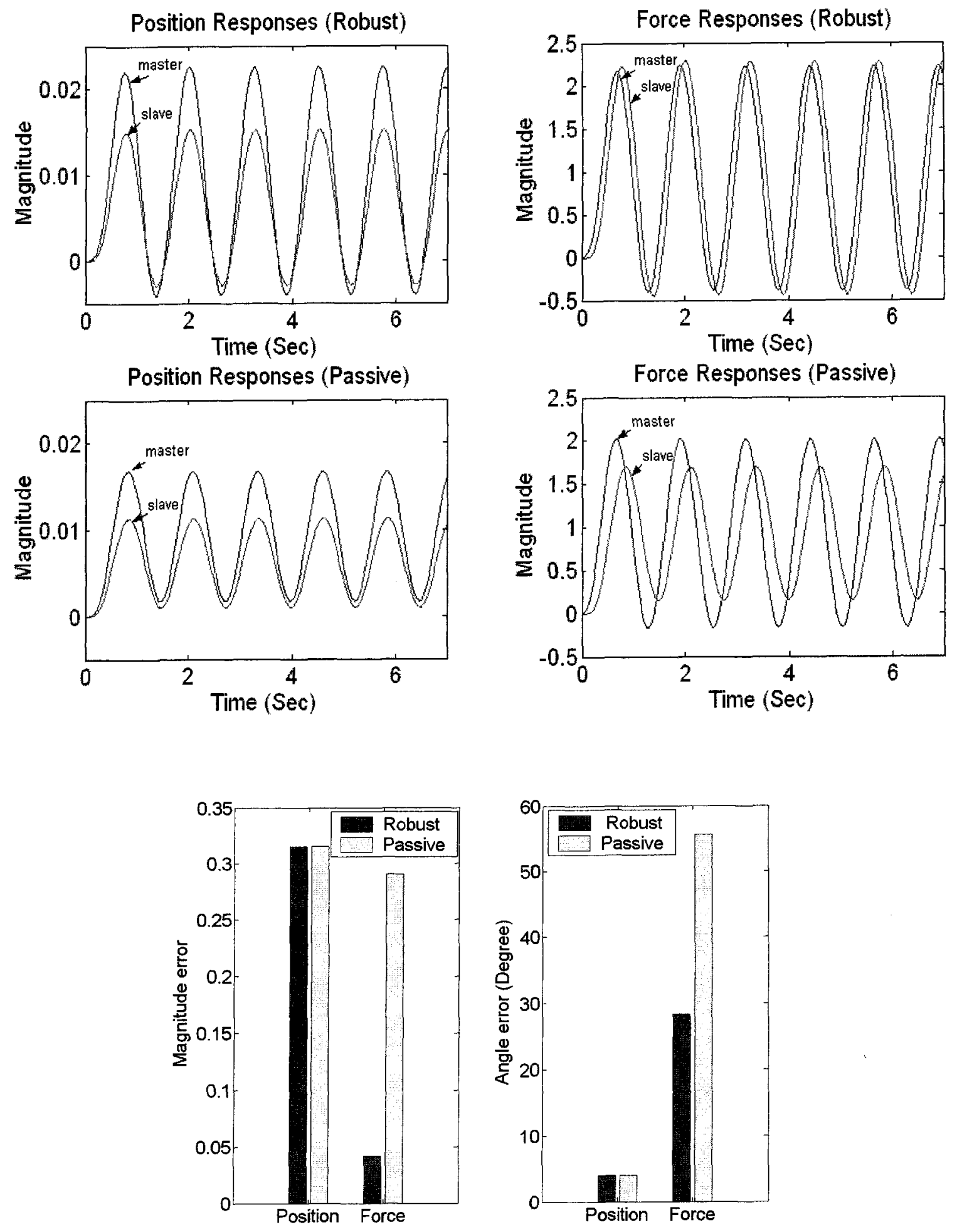

Figure 4.9 (b): Frequency $=0.8 \mathrm{~Hz}$. 

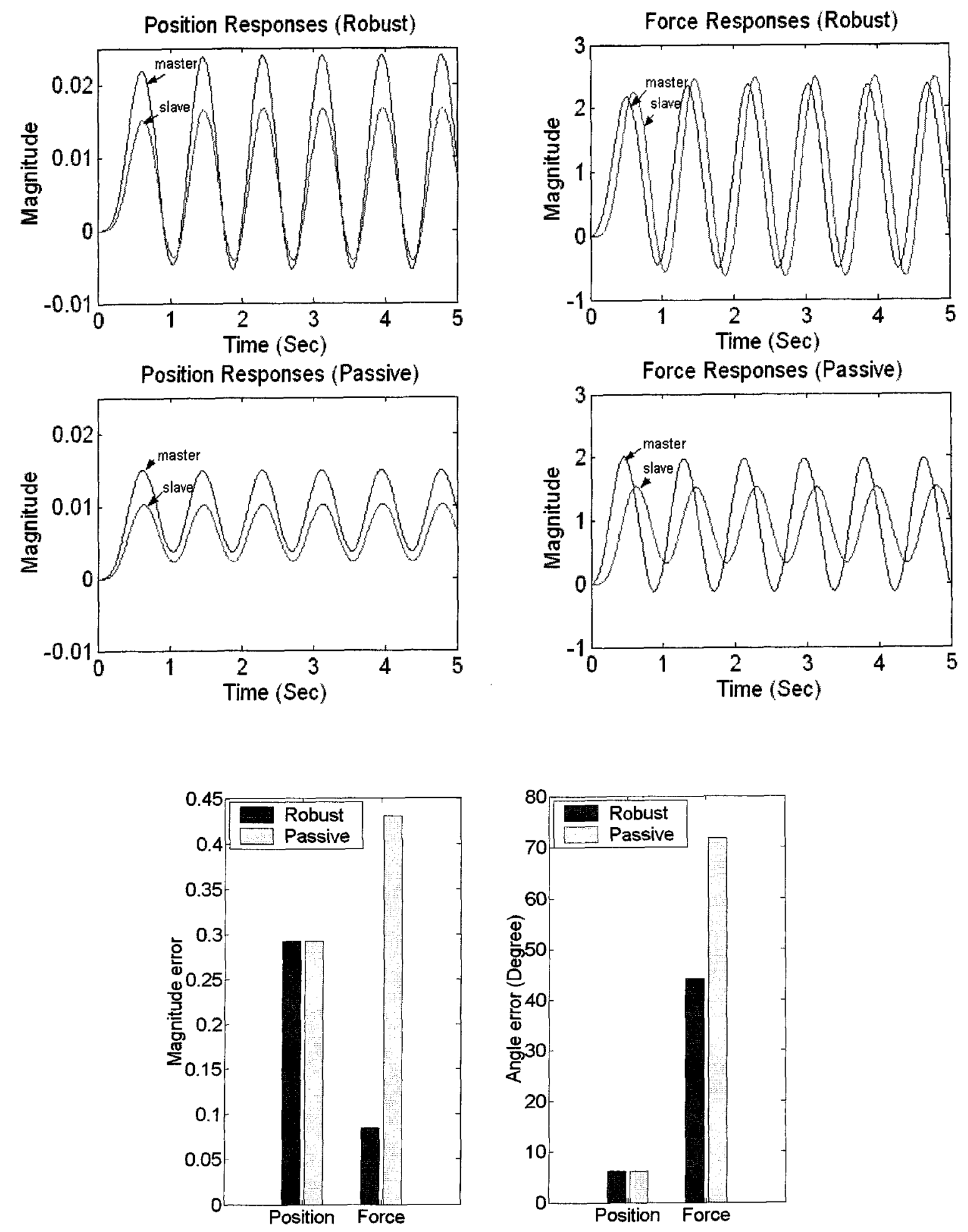

Figure 4.9 (c): Frequency $=1.2 \mathrm{~Hz}$. 

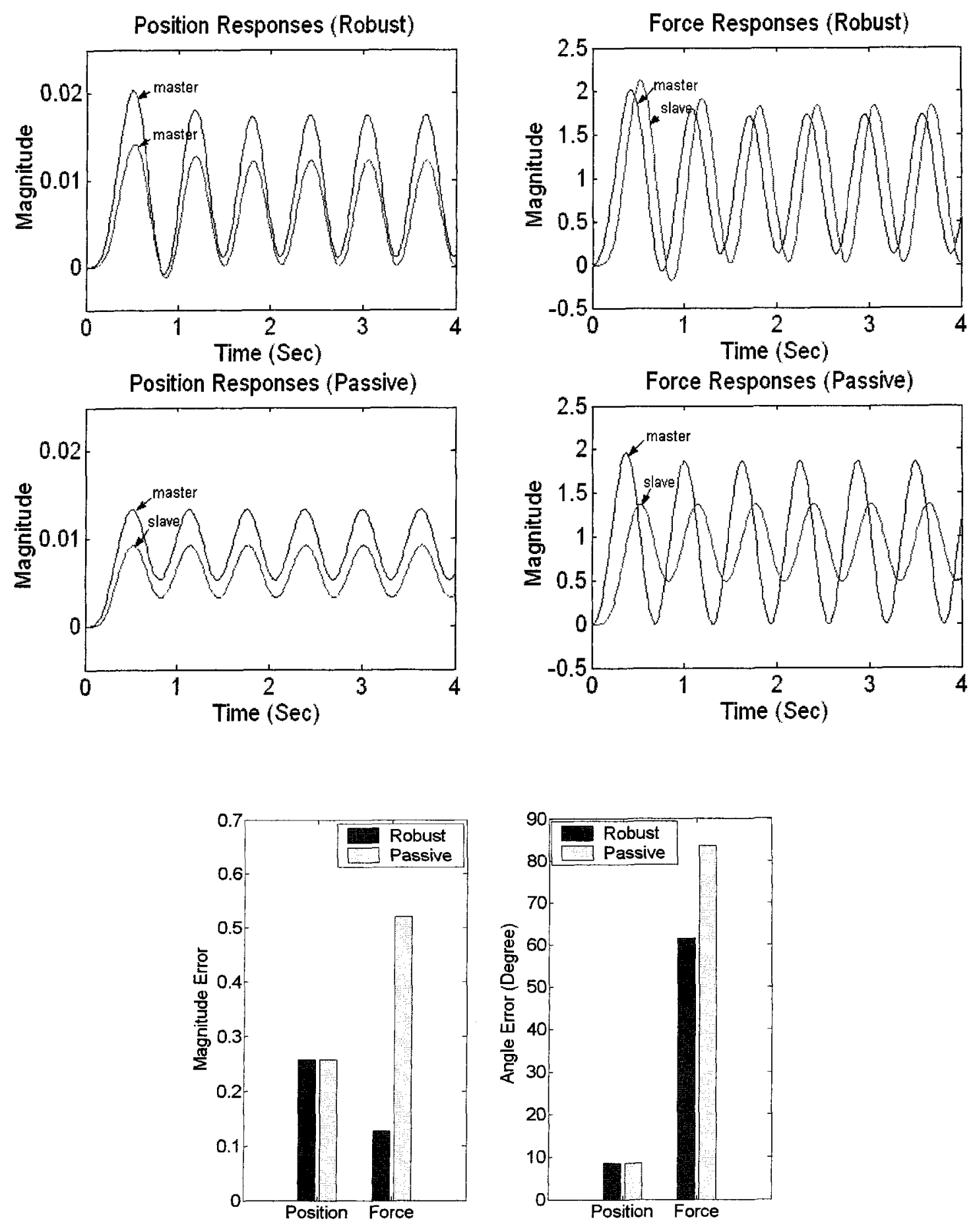

(d): Frequency $=1.6 \mathrm{~Hz}$.

Figure 4.9: Fidelity comparisons. 


\subsection{Modified Position-Force Controlled Teleoperator (Case 2)}

In chapter 2 , the dynamics of the modified position-force controlled structure were analyzed and the two-port hybrid matrix was derived. For this structure, the local force feedback gain $\alpha$ and the position error gain $g$ are the parameters that can be controlled. We have a prescribed parameter range of $g \in[0,300]$ and $\alpha \in[0,1]$ respectively.

In this case study, most physical model parameters are the same as those in the previous case. For the human operator, we have $m_{h}=1.5 \mathrm{~kg}, b_{h}=1.0 \mathrm{Ns} / \mathrm{m}$ and $k_{h}=8 \mathrm{~N} / \mathrm{m}$; the master and slave robots' inertias are $m_{m}=m_{s}=0.5 \mathrm{~kg}$; the master manipulator's damping ratio is $b_{m}=15 \mathrm{Ns} / \mathrm{m}$, and the slave's damping ratio is still $b_{s}=6 \mathrm{Ns} / \mathrm{m}$; the stiffness of master and slave are $k_{m}=k_{s}=0 \mathrm{~N} / \mathrm{m}$; the nominal environment impedance is $Z_{e 0}=k_{e 0}=150 \mathrm{~N} / \mathrm{m}$; the environment impedance uncertainty range is still $Z_{e}=k_{e} \in[50,300] N / m$.

\subsubsection{Tracking Constraint}

We know that the transfer function for tracking error is the sensitivity function, which is given by equation (3.3.3). That is

$$
S_{e n}(s)=1+H_{21}(s)
$$

From, equation (2.2.22), we know that for the modified position-force structure 


$$
H_{21}(s)=-\frac{g}{m_{s} s^{2}+b_{s} s+k_{s}+g}
$$

Then we have

$$
S_{e n}(s)=1+H_{21}(s)=\frac{m_{s} s^{2}+b_{s} s+k_{s}}{m_{s} s^{2}+b_{s} s+k_{s}+g}
$$

This sensitivity function is the same as the one in the previous case. The input signal considered here is the master position $X_{m}(s)$ and the output is the position tracking error between the master and slave, i.e. $X_{m}(s)+X_{s}(s)$.

The difference between this modified position-force structure and the position-force controlled structure in the previous case is that, in the second case, the forward position error gain $g$ is a controller parameter rather than a fixed parameter and $S_{e n}(s)$ is a function of $g$. So, we need to first find out the free space tracking constraint for the controller parameter $g$.

We still use the performance requirement of the previous case study, i.e. the error between the master and slave manipulators should be less than $10 \%$ of the input signal $X_{m}$, which is a unit sinusoid with frequency lower than $0.5 \mathrm{~Hz}$.

The Bode plots for the transfer functions $S_{e n}(s)$ with different values of $g$ are shown in Figure 4.10. The dashed lines in the figure specify the performance requirement and the solid curves represent frequency responses of the sensitivity functions $S_{e n}(s)$ with different values of $g$. According to equation (3.3.5), if the frequency response lies below the dashed lines, the tracking constraint is satisfied. 


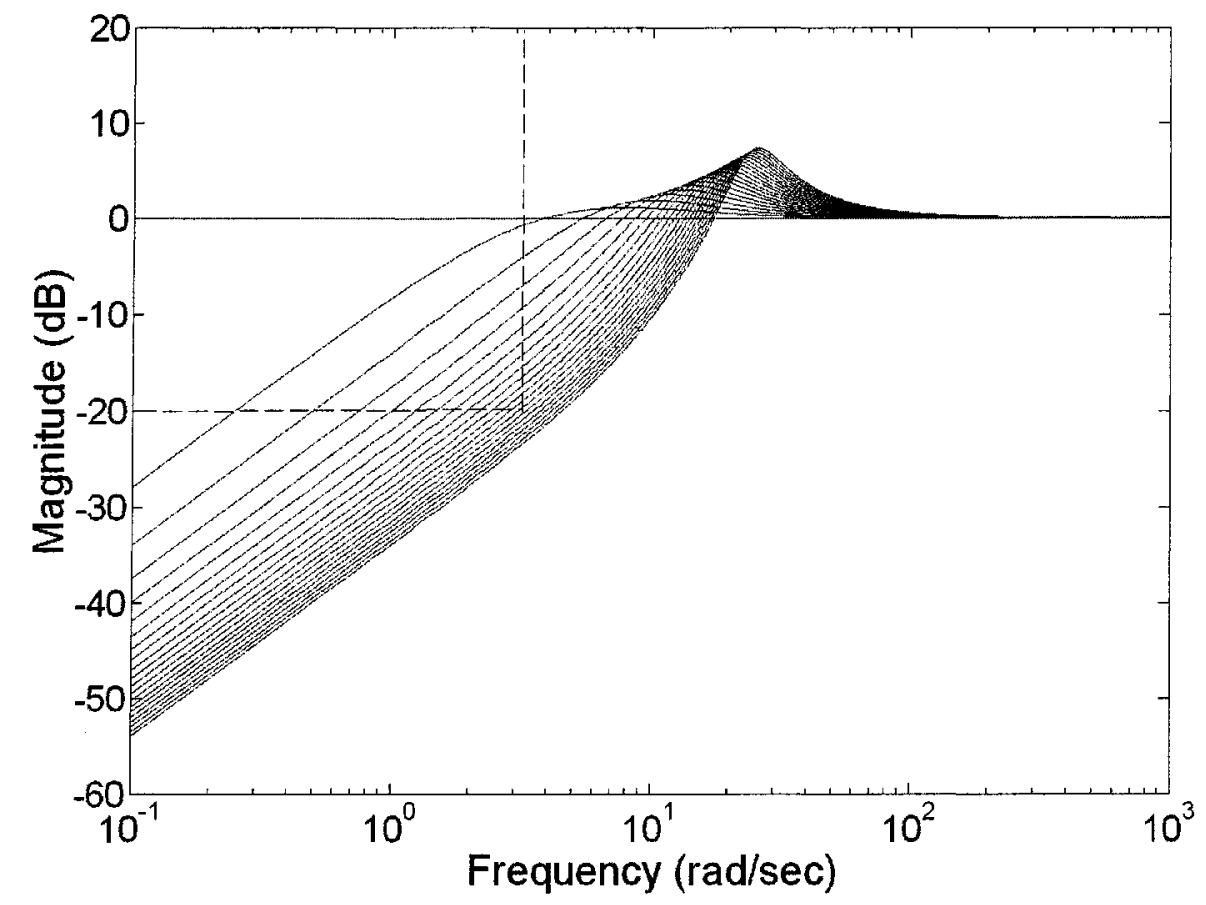

Figure 4.10: Tracking performance constraint (Case 2).

For the current system configuration, it was found that the tracking performance specification is satisfied only when $g \geq 220$. As the prescribed range for $g$ is $[0,300]$, the new constraint for $g$ is $[220,300]$. Note that the constraint for $g$ does not affect the other controller parameter $\alpha$, which is still in the range $[0,1]$.

\subsubsection{Robust Stability Analysis}

\section{Stability of Nominal System}

Now we check the stability of the nominal system. The open loop gain $G_{0}(s)$ of the system in the nominal environment can be obtained by equation (3.4.10), that is 


$$
G_{0}(s)=\frac{-H_{12}(s) H_{21}(s) Z_{e 0}(s)}{\left[H_{11}(s)+Z_{h}(s)\right]\left[1+H_{22}(s) Z_{e 0}(s)\right]}
$$

The closed-loop transfer function is

$$
\hat{G}(s)=\frac{G_{0}(s)}{1+G_{0}(s)}
$$

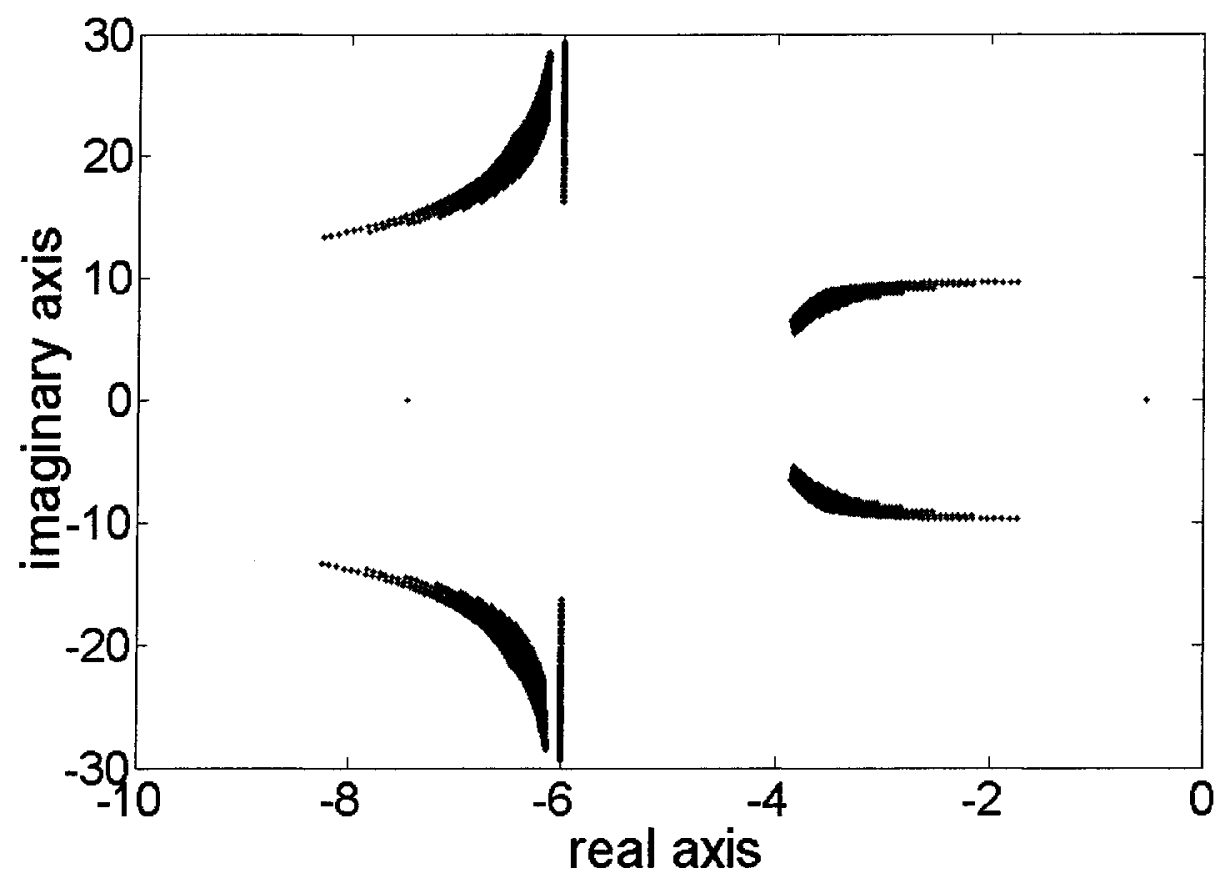

Figure 4.11: Nominal closed-loop system poles (Case 2).

According to equation (3.4.10) and (2.2.22), we know that $\hat{G}(s)$ is a function of both $g$ and $\alpha$. Figure 4.11 shows the plot of poles of $\hat{G}(s)$ for $g \in[220,300]$ and $\alpha \in[0,1]$. It can be seen that all the closed-loop poles of the nominal plant are located in the left half of the complex plane. In other words, the nominal closed loop system is always stable for the controller parameters $g \in[220,300]$ and $\alpha \in[0,1]$. 


\section{Finding the Bounding Function}

As in the first case, we need to find a bounding function for the environment uncertainty. From equation (3.4.12), we know that this uncertainty function is only related to $H_{22}(s)$ of the hybrid matrix.

For the modified position-force based structure, from equation (2.2.22), we have

$$
H_{22}(s)=\frac{1-\alpha}{m_{s} s^{2}+b_{s} s+k_{s}+g}
$$

Different from the first case study, the uncertainty term $W_{d}(s)=W(s) \Delta(s)$ described in equation (3.4.12) contains both controller parameters $g$ and $\alpha$. This means that finding the bounding function in this situation will be more difficult than in the first case study. Since an analytical result of $W(s)$ as a function of the controller parameters are often impossible to be achieved, we need to divide the controller parameters' ranges into segments and derive the bounding function for each segment. This approach can avoid getting too conservative results of $W(s)$. In this case study, we separate $\alpha$ into two segments, $\alpha \in[0,0.5]$ and $\alpha \in(0.5,1]$. The other parameter is $g \in[220,300]$, which is not divided. Of course, if we divide the parameters' range into more segments, the result will be more accurate.

First, let us consider the parameter range $\alpha \in[0,0.5]$ and $g \in[220,300]$. By systematically varying the environment impedance $Z_{e}$, in its uncertainty range $Z_{e} \in[50,300] N / m$ and varying $\alpha$ and $g$ simultaneously, we can find the bounding function according to the analysis of subsection 3.4.3. The uncertainty transfer functions 
$W_{d}(s)=W(s) \Delta(s)$ are shown in Figure 4.12.

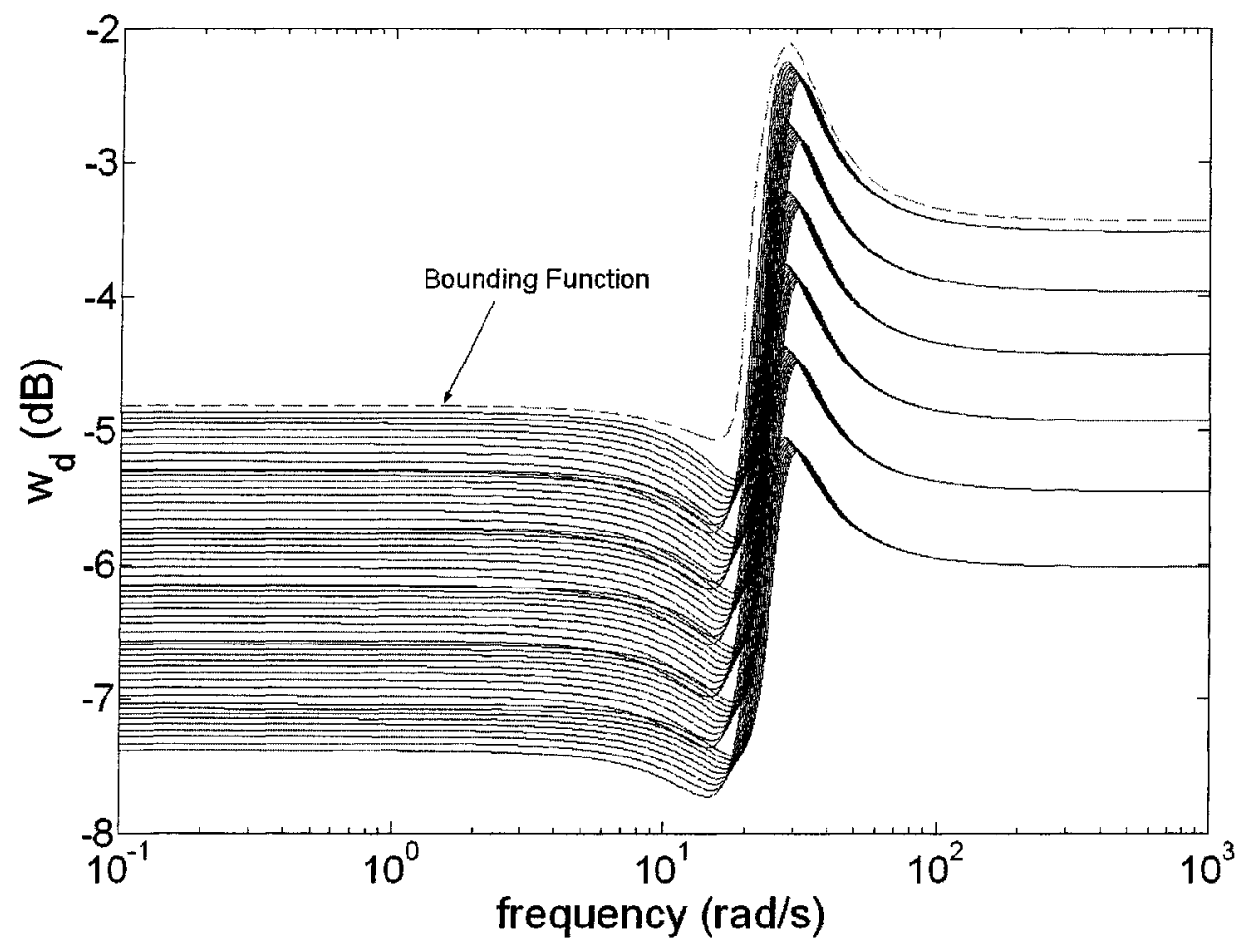

Figure 4.12: Find the bounding function (Case 2, $\alpha \in[0,0.5]$ ).

Each solid curve on the above diagram corresponds to specific impedance and the curves are plotted according to equation (3.4.12). The dashed curve above the top of the set of solid curves is the bounding function $W_{\alpha \in[0,0.5]}(s)$.

Following the same procedure of the first case study, the following bounding function is given,

$$
W_{\alpha \in[0.5,1]}(s)=\frac{0.6696 s^{4}+22.32 s^{3}+771.2 s^{2}+8959 s+137300}{s^{4}+31.53 s^{3}+1218 s^{2}+14110 s+238700}
$$

For $\alpha \in(0.5,1]$ and $g \in[220,300]$, the same steps as for the case of $\alpha \in[0,0.5]$ 
are taken. The uncertainty transfer functions $W_{d}(s)=W(s) \Delta(s)$ are shown in Figure 4.13.

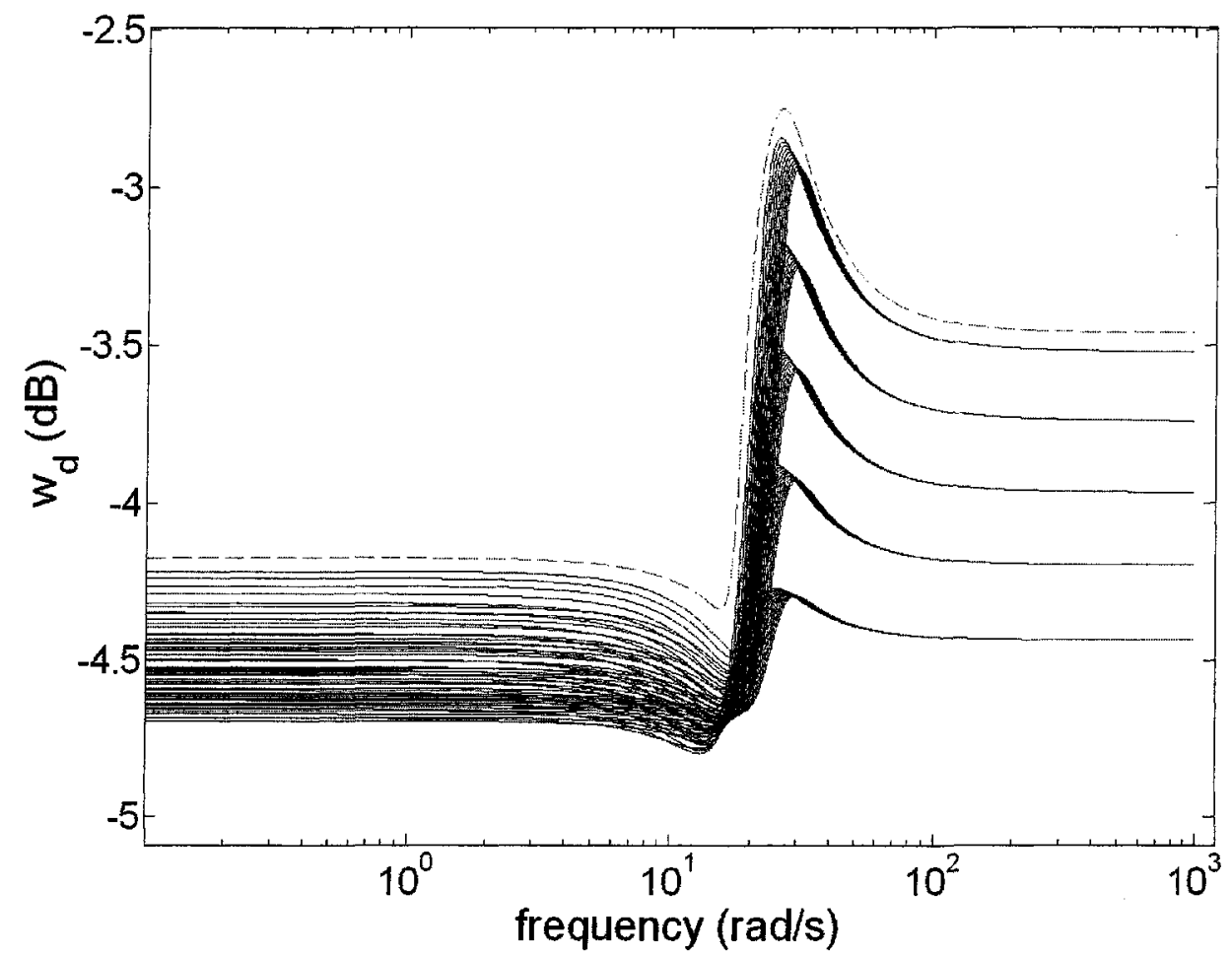

Figure 4.13: Find the bounding function (Case 2, $\alpha \in(0.5,1])$.

Each solid curve in the above diagram corresponds to specific impedance. The dashed curve on top of the set of solid curves is the bounding function $W_{\alpha \in(0.5,1]}(s)$. We have,

$$
W_{\alpha \in(0.5,1]}(s)=\frac{0.6713 s^{4}+14.22 s^{3}+615.7 s^{2}+5241 s+112200}{s^{4}+20.55 s^{3}+955.5 s^{2}+7897 s+181500}
$$

\section{Internal Stability Analysis}

So far we have found the bounding functions for the system design. Then the controller 
parameters that can maintain internal stability of the system can be found. Rewriting equations (3.4.13) and (3.4.10), we have

$$
T(s)=1-S(s)=\frac{G_{0}(s)}{1+G_{0}(s)}
$$

where $G_{0}(s)$ is defined in equation (3.4.10).

The internal stability function $\|T(s) W(s)\|_{\infty}$ for $\alpha \in[0,0.5]$ and $g \in[220,300]$ is shown in Figure 4.14. In the figure, the stability function value $\left\|T(s) W_{0.5}(s)\right\|_{\infty}$ determines the internal stability of the system. As mentioned in Theorem 3.1, when $\|T(s) W(s)\|_{\infty} \leq 1$, the system is stable. It is seen that if the controller parameters $g \in[220,300]$ and $\alpha \in[0,0.5]$, the above requirement is always satisfied and thus the system is always internally stable.

Similarly, for $\alpha \in(0.5,1]$ and $g \in[220,300]$, we have Figure 4.15. In the figure, the stability requirement is not always satisfied. The bold solid line on the surface shows the contour of $\|T(s) W(s)\|_{\infty}=1$. The corresponding internally stable region for the two controller parameters is shown in Figure 4.16.

So, from the above analysis, we know that if $\alpha \in[0,0.5]$ and $g \in[220,300]$, the system is always stable; if $\alpha \in(0.5,1]$ and $g \in[220,300]$, the system stability can be determined according to the stability region shown in Figure 4.16. 


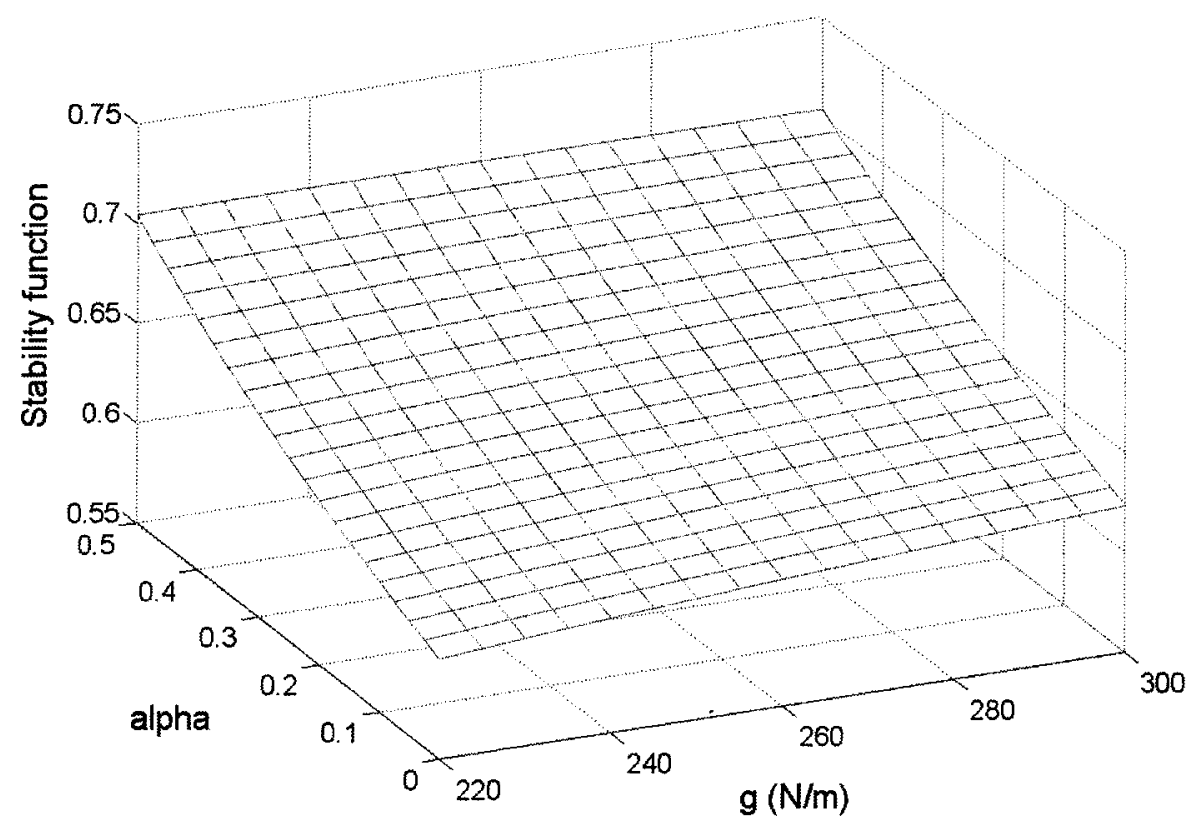

Figure 4.14: Internal stability criterion (Case 2, $\alpha \in[0,0.5]$ ).

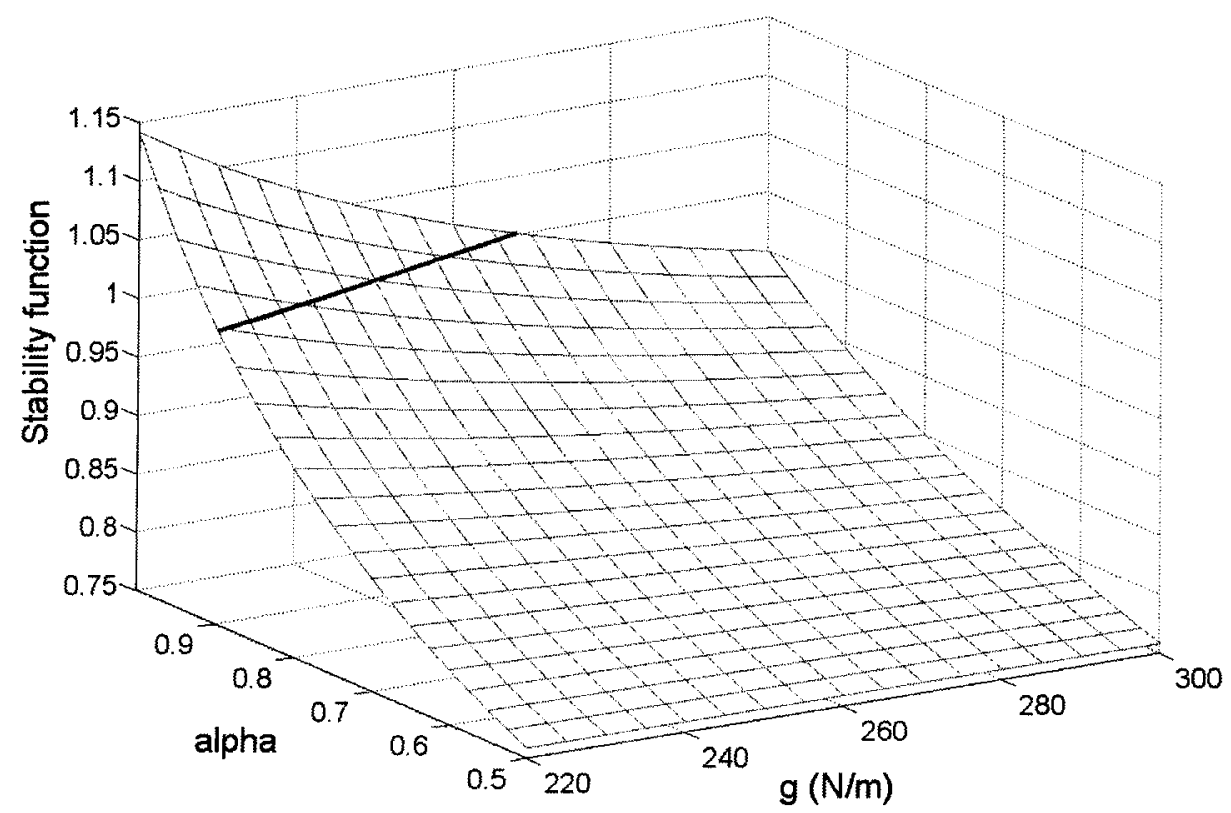

Figure 4.15: Internal stability criterion (Case $2, \alpha \in(0.5,1]$ ). 


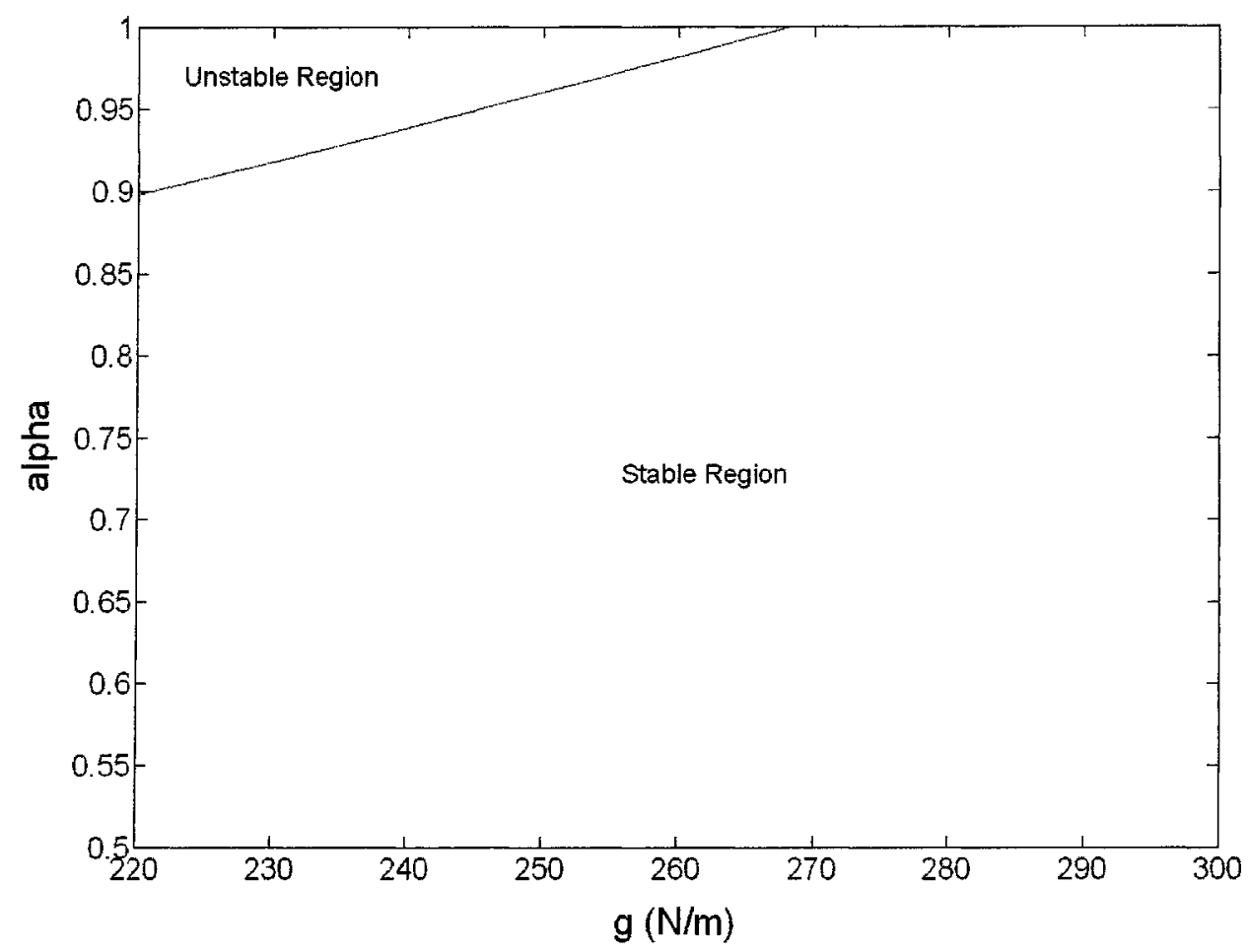

Figure 4.16: Internal stability region (Case 2).

\subsubsection{Fidelity Evaluation}

At this point, the parameter range that satisfies both stability and tracking constraint has already been found. The next design step is to calculate the controller parameter value that maximizes the system fidelity, i.e., find parameters $g$ and $\alpha$ that minimizes the fidelity evaluation function value $D_{z}$. From equation (3.2.3), the impedance transmitted to the human operator for a SISO system is

$$
Z_{t}(s)=\frac{F_{m}(s)}{X_{m}(s)}=H_{11}(s)-\frac{H_{12}(s) H_{21}(s) Z_{e 0}(s)}{1+H_{22}(s) Z_{e 0}(s)}
$$

From equation (3.2.3) and (2.2.22), it can be found that the above equation is a 
function of $g$ and $\alpha$. Thus we can find a value for controller parameters $g$ and $\alpha$ that minimizes the fidelity measure function $D_{z}\left(Z_{e 0}\right)=\left\|\left[Z_{t}(s)-Z_{e 0}(s)\right] \cdot l(s)\right\|_{\infty} \cdot l(s)$ was already defined in equation 4.2.3.

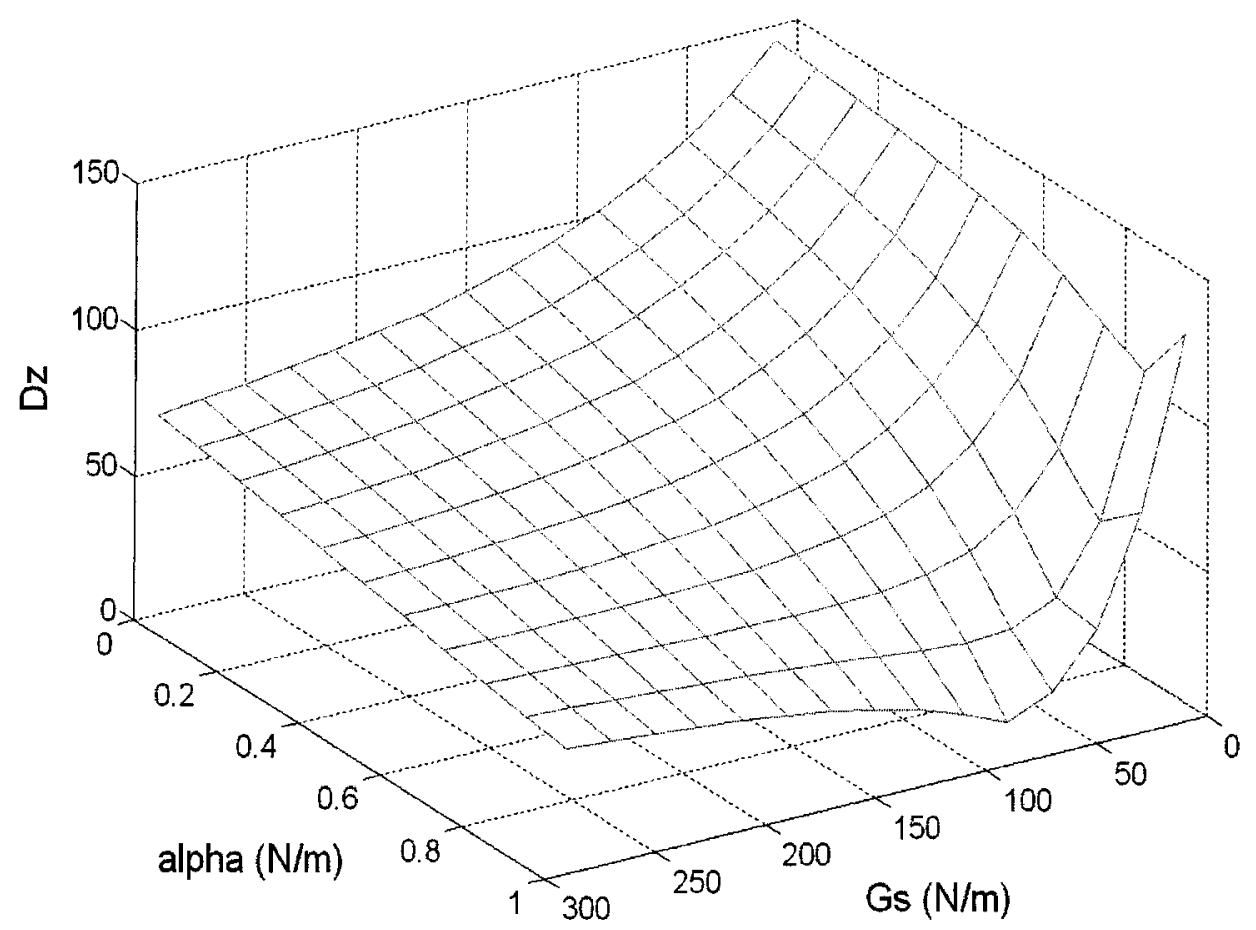

Figure 4.17: Fidelity evaluation function.

The fidelity function value is depicted in Figure 4.17 as a function of $g$ and $\alpha$. The best performance is achieved when the minimal fidelity function value $D_{z}$ is achieved. This minimal fidelity function corresponds to $\alpha=0.98$ and $g=260$, with $D_{z}=41.7$. Such a parameter pair can satisfy the robust stability constraint, free-space tracking constraint and achieves the best fidelity. This completes our robust design 
procedure.

\subsubsection{Design Based on Passivity Theorem}

As mentioned in the previous case study, the infinity norm of the scattering matrix, $\left\|S_{c}(s)\right\|$, can be used to determine the passivity of the system. Recall that the scattering matrix is defined by

$$
S_{c}(s)=\left[\begin{array}{cc}
1 & 0 \\
0 & -1
\end{array}\right](h(s)-I)(h(s)+I)^{-1}
$$

We already know how to calculate the matrix $h(s)$ from equation (3.4.6), we can determine the system passivity.

$\left\|S_{c}(s)\right\|_{\infty}$ is shown as a function of $g$ and $\alpha$ in Figure 4.18. The corresponding passive region is shown in Figure 4.19. It was found that the best fidelity for parameters in the passivity region is achieved when $\alpha=0.11$ and $g=300$, with $D_{z}=65.7$. This value is much larger than the best fidelity value of the robust design with $D_{z}=41.7$. So, the fidelity of the robust design is better than the passive design 


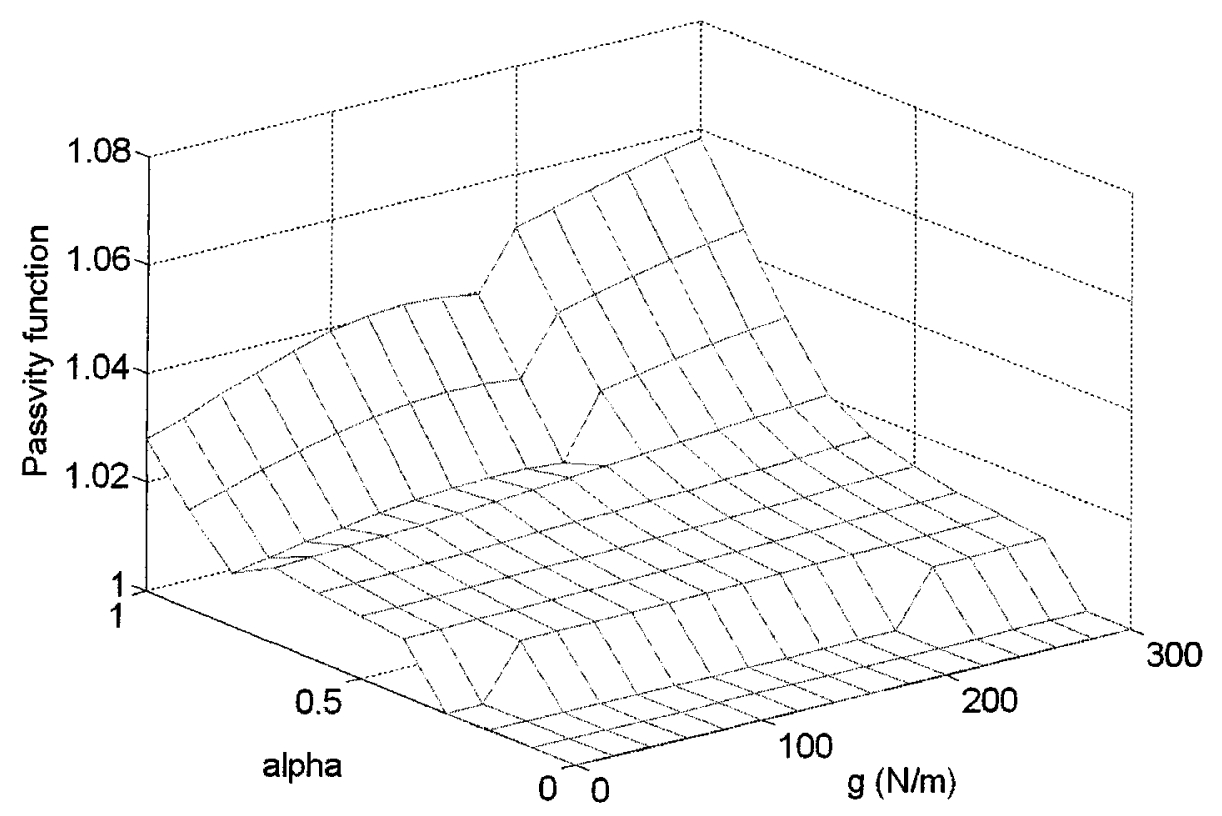

Figure 4.18: Stability analysis based on passivity.

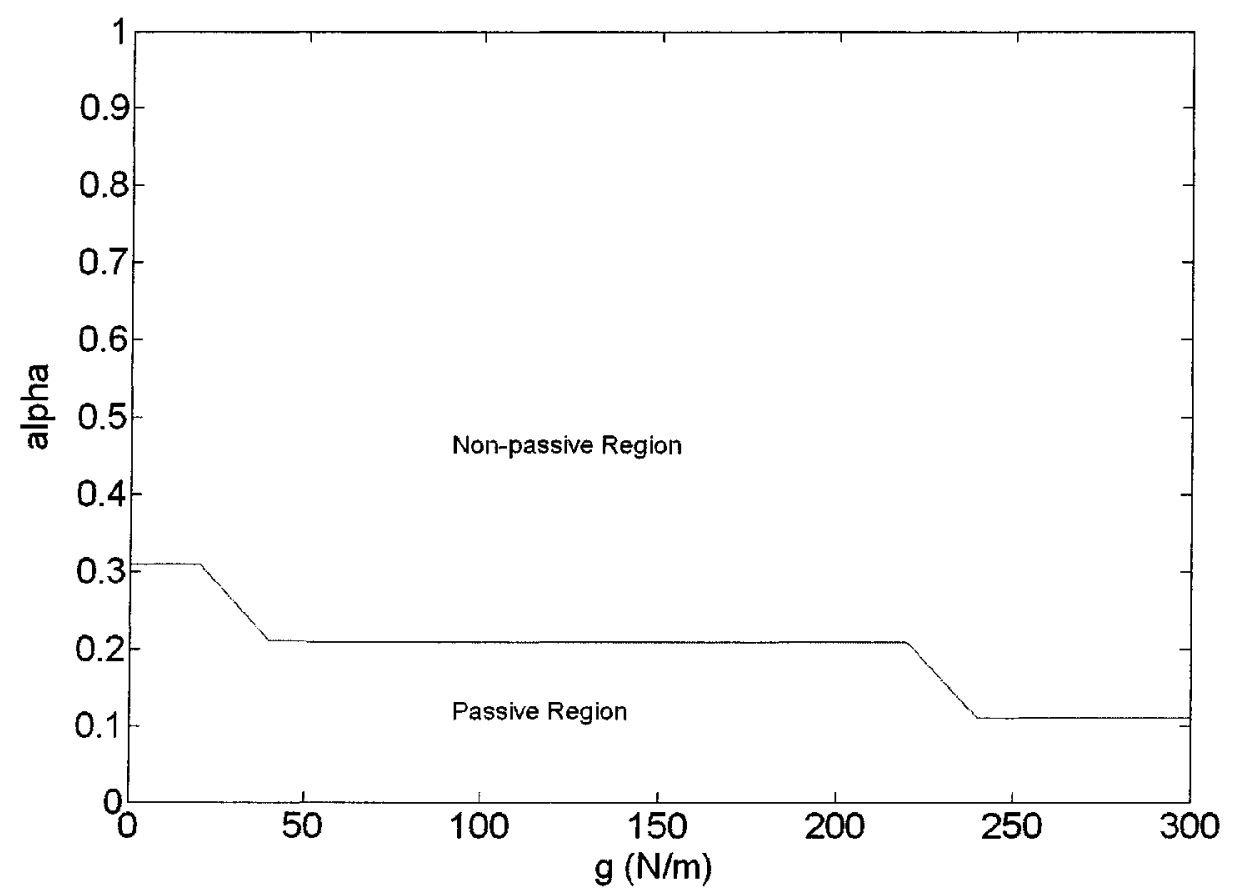

Figure 4.19: Passive region (Case2). 


\subsubsection{Fidelity Comparison}

We can summarize the design results: for robust control design, the best fidelity can be achieved when the controller parameters are $\alpha=0.98$ and $g=260$ and, for passivity design, the best fidelity is achieved when the controller parameters are $\alpha=0.11$ and $g=300$. We can compare these two situations.

Same as in the previous case study, the fidelity comparison can be conducted by showing all four force and position responses of both the master and slave manipulators.

The position and force responses are shown in Figure 4.20. In the figure, the position and force error magnitudes and phases are calculated and compared at different frequencies. The position and force errors are relative error between the master and slave, i.e. $\operatorname{err} x=\left(x_{m}+x_{s}\right) / x_{m}$ and errf $=\left(f_{m}-f_{s}\right) / f_{m}$. The input signal is $f_{h}=1-\cos \omega t$. The input signal frequencies are chosen to be $0.4 \mathrm{~Hz}, 0.8 \mathrm{~Hz}, 1.2 \mathrm{~Hz}$, and $1.6 \mathrm{~Hz}$.

From Figure 4.20, it was observed that for all four input frequencies, the force and position magnitude tracking errors between the master and slave of robust design are much smaller than that of passive design; the force angle error of robust design is smaller than the robust design and the position angle error of robust design is a little larger than the robust design. As in the previous case study, the results show that the fidelity is greatly improved when the robust design method is applied and the new fidelity function is validated. 

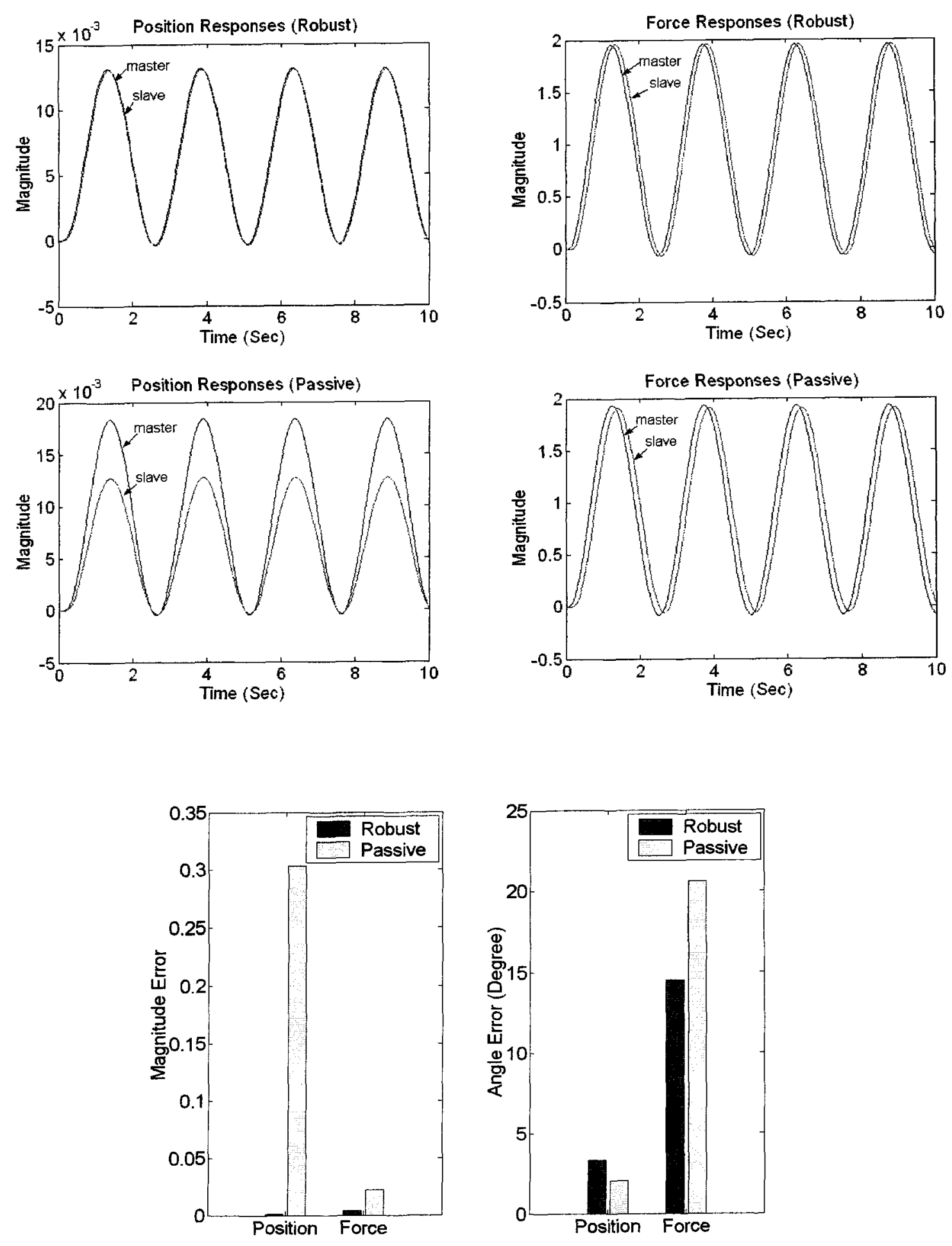

Figure 4.20 (a): Frequency $=0.4 \mathrm{~Hz}$. 

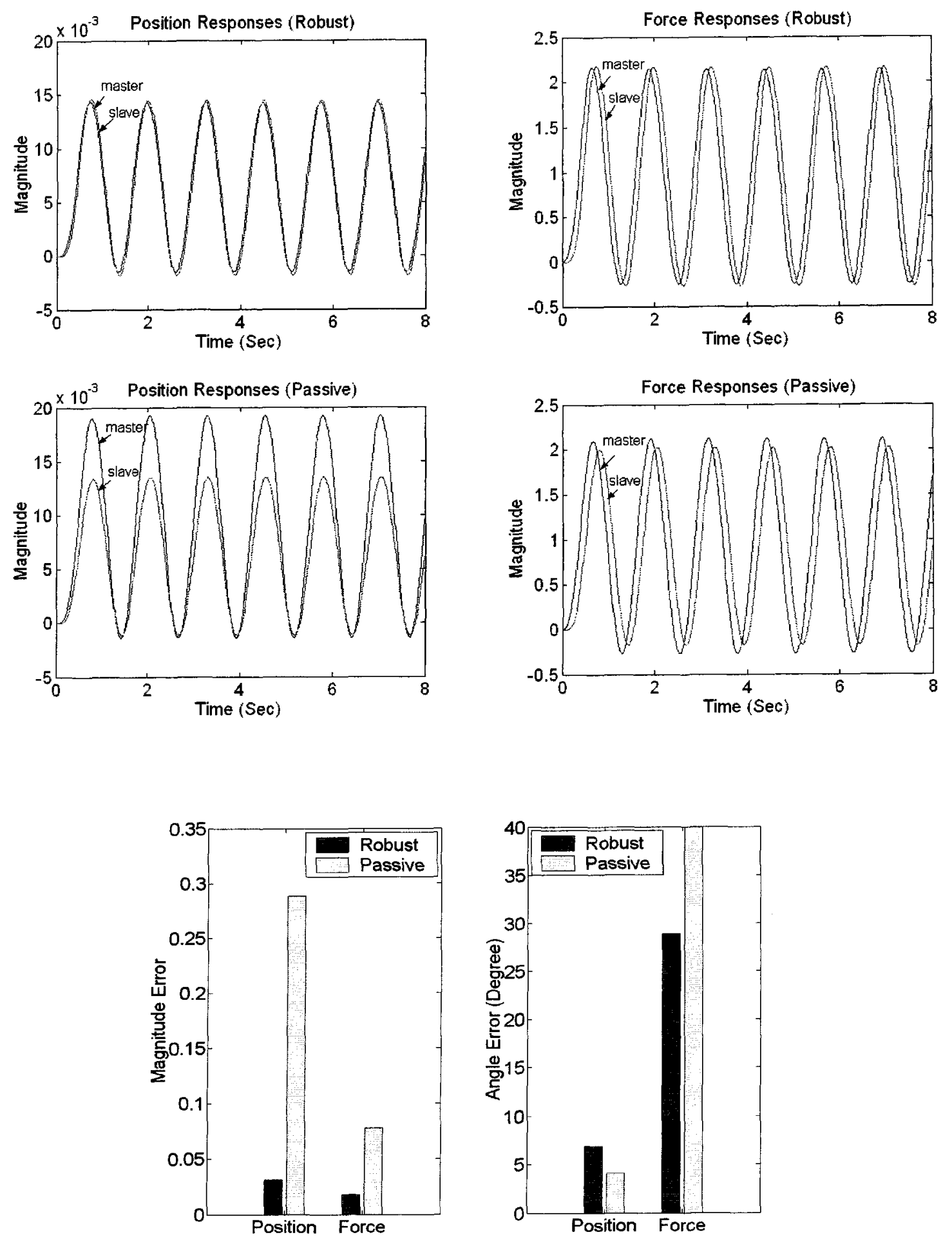

Figure $4.20(b)$ : Frequency $=0.8 \mathrm{~Hz}$. 

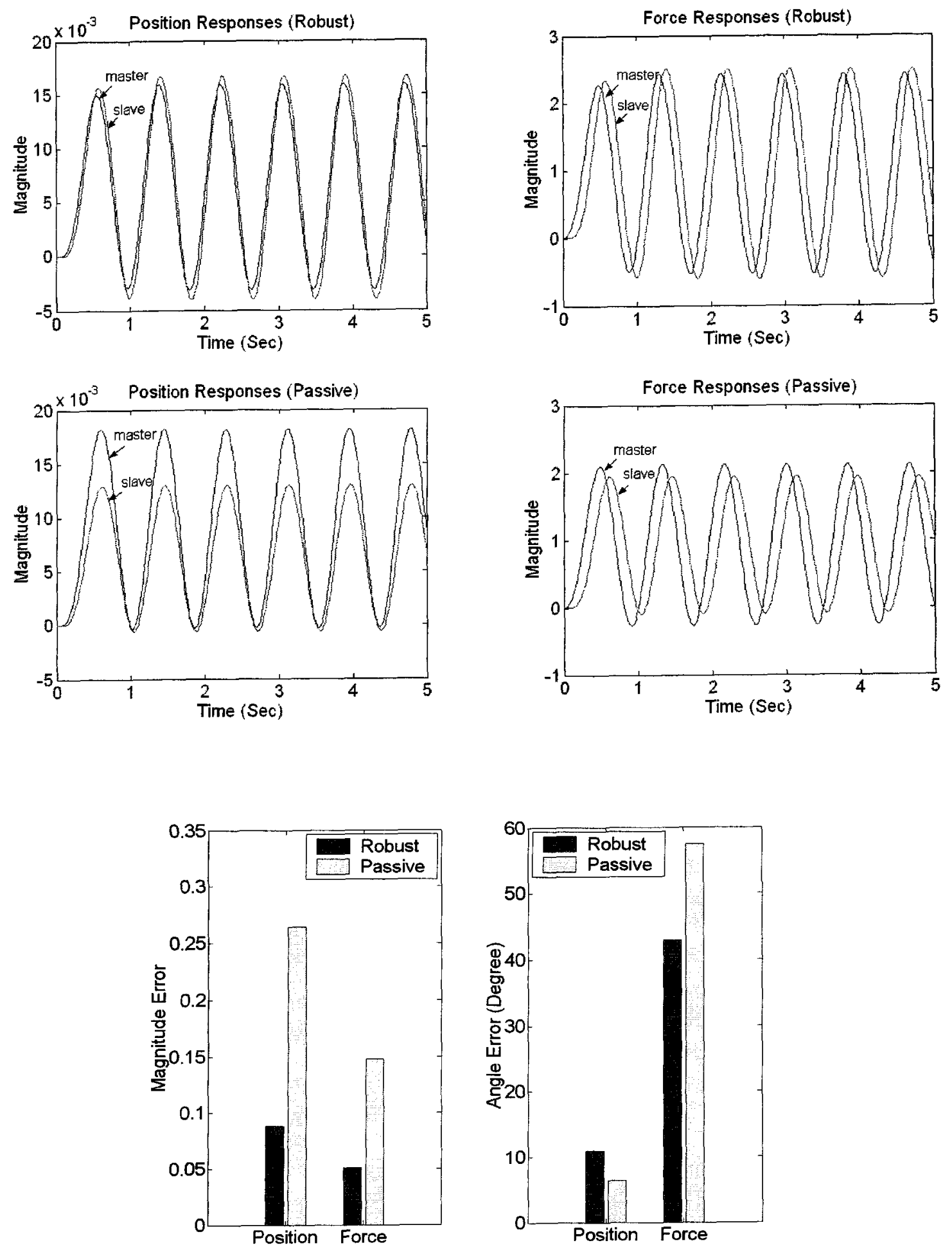

Figure $4.20(\mathrm{c}):$ Frequency $=1.2 \mathrm{~Hz}$. 

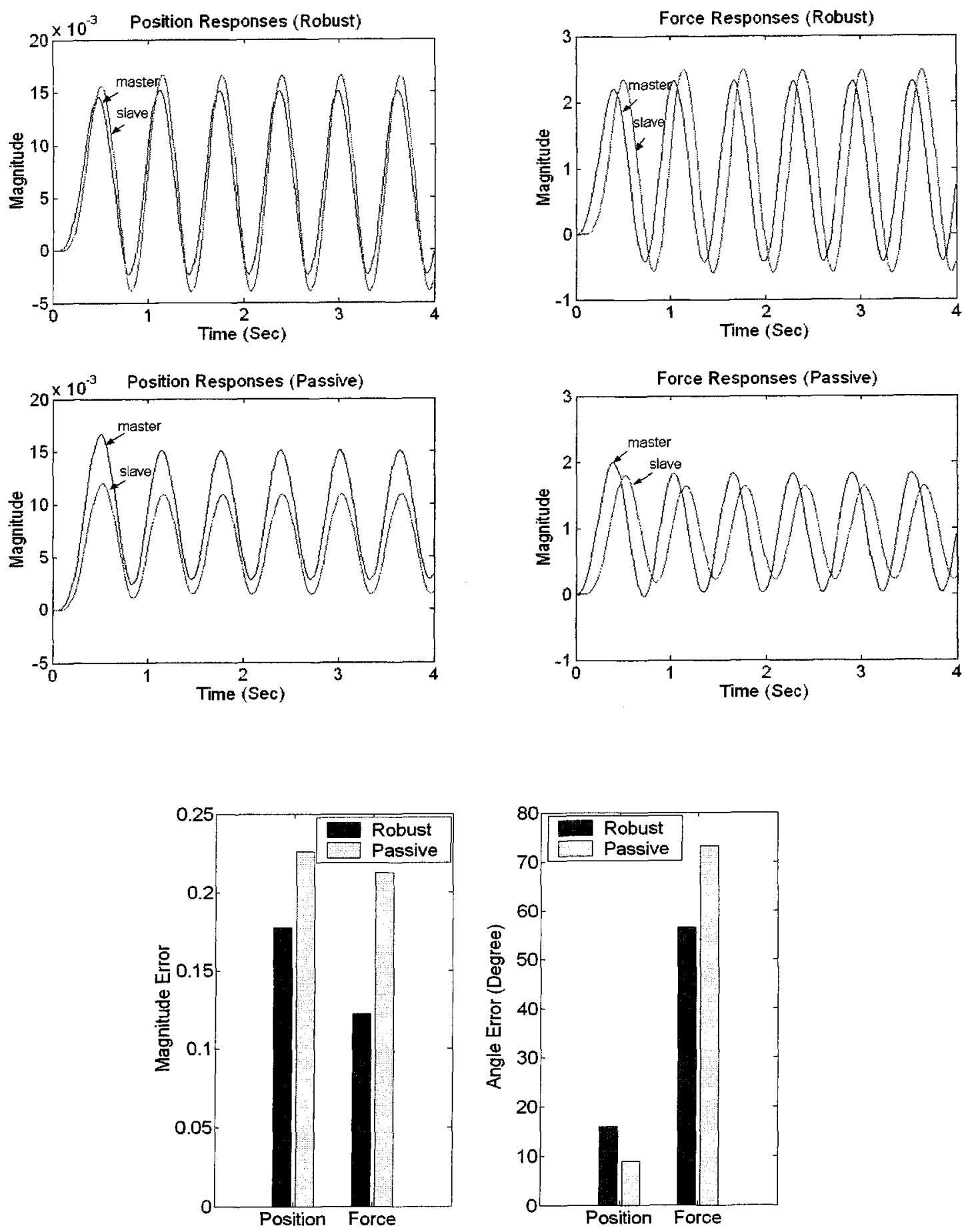

(d) Frequency $=1.6 \mathrm{~Hz}$.

Figure 4.20: Fidelity comparison (Case 2). 


\section{Chapter 5}

\section{Conclusions}

In this thesis, research on improving the performance of bilateral teleoperators under the constraint of stability with soft environments was studied. A new fidelity measure was proposed and a robust controller design method was applied on bilateral teleoperators. Two case studies were performed to show the design process and fidelity comparison of the proposed design approach and the traditional passivity based design approach.

This thesis targets on the application of telesurgery. Although much work has been done in the teleoperation field in the past decade, due to the technical limitations, the application of telesurgery system is still in its infancy. Since surgeons' kinaesthetic sensation is vital to the success of a surgical operation, the fidelity requirement of telesurgery systems is much higher than that of the traditional teleoperation systems. In traditional systems, the stability problem is more significant due to the stiff environment. People have to sacrifice performance to achieve better stability margin. However, in 
telesurgery applications, since the environment is normally soft, the stability constraint is less rigid. So we use robust control to improve the performance or fidelity of the system. The case studies introduced in chapter 4 , validate the feasibility and effectiveness of the proposed design approach.

There are still a few problems that need to be explored in the future research. The first one is to detect the environment characteristics. For example, how to determine the range of environment impedance uncertainty range is an open issue. If the range is too narrow, the fidelity of the system cannot be satisfactory. The characteristics of different surgical environments should be classified and investigated in the future and such data are critical to the application of telesurgery.

The second problem is the safety problem. Telesurgery sets a very high level of safety requirement since it is directly related to the health or even life of the patients. Generally, supervisory control should be applied to provide safe operations. It enables the system to be continuously supervised and prevented from performing any unsafe operations.

Finally, in this thesis, communication channel characteristics and time delay are not considered. This does not mean it is not important for the system design. In fact, a small time delay may cause an originally stable system to become unstable. Furthermore, for Internet-based teleoperators, the communication time delay is usually random in nature and changes abruptly and this increases the difficulty of system design. 


\section{References}

[1] R. J. Adams and B. Hannaford. Stable haptic interaction with virtual environments, IEEE. Transactions on Robotic and Automation, vol. 15, issue 3, pp. 465-474, June 1999

[2] I. Aliaga, A. Rubio, and E. Sanchez. Experimental Quantitative Comparison of Different Control Architectures for Master-slave teleoperation, IEEE Transactions on Control Systems Technology, vol. 12, issue 1, pp. 2-11, Jan. 2004

[3] R. J. Anderson and M. W. Spong. Bilateral control of teleoperators with time delay, IEEE Transactions on Automatic Control, vol. 34, issue 5, pp. 494-501, May 1989

[4] F. Arai, M. Tanimoto, T. Fukuda, K. Shimojima, H. Matsuura, and M. Negoro. Multimedia tele-surgery using high speed optical fiber network and its applications to intravascular neurosurgery - system configuration and computer networked implementation, Proceedings of the 1996 IEEE International Conference on Robotics and Automation, vol. 1, pp. 878-883, 1996

[5] T. L. Brooks. Telerobotic response requirements, Proceedings of the 1990 IEEE International Conference on Systems, Man and Cybernetics, pp. 113-120, Nov. 1990

[6] J. R. Burnett. Force reflecting-servos add "feel" to remote controls, Control Engineering, vol. 4, no. 5, pp.1269-1274, 1957

[7] M. C. Cavusoglu, A. Sherman, and F. Tendick. Design of bilateral teleoperation 
controllers for haptic exploration and telemanipulation of soft environments, IEEE Transactions on Robotics and Automation, vol. 18, issue 4, pp. 641-647, Aug. 2002

[8] J. E. Colgate. Robust impedance shaping telemanipulation, IEEE Transactions on Robotics and Automation, vol. 9, issue 4, pp. 374-384, Aug. 1993

[9] J. E. Colgate and N. Hogan. Robust control of dynamically interacting systems, International Journal of Control, vol. 48, no. 1, pp. 65-88, 1988

[10] R. W. Daniel and P. R. MacAree. Fundamental limits of performance for force reflecting teleoperation, International Journal of Robotics Research, vol. 17, no 8, pp. 811-830, Aug. 1998

[11] C. A. Desoer and M. Vidyassagar. Feedback systems: Input Output Properties, Academic Press, 1975

[12] J. Dudragne. A generalized bilateral control applied to master-slave manipulators, Proceedings of the $20^{\text {th }}$ International Symposium on Industrial Robots, pp. 435-442, 1989 [13] K. B. Fite, L. Shao, and M. Goldfarb. Loop shaping for transparency and stability robustness in bilateral telemanipulation, IEEE Transactions on Robotics and Automation, Vol. 20, No.3, pp. 620-624, June 2004

[14] K. B. Fite, J. Speich, and M. Goldfarb. Transparency and stability in two-channel bilateral telemanipulation. ASME Journal of Dynamic systems, Measurement, and Control, vol. 123, pp. 400-407, Mar. 2001

[15] B. Francis, $A$ course in $H_{\infty}$ control theory, Springer-Verlag, 1987

[16] M. Ghodoussi, S. E. Butner, and Y. Wang. Robotic Surgery- the transatlantic case, Proceedings of the 2002 IEEE International Conference on Robotics and Automation, vol. 
2, pp. 1882-1888, May 2002

[17] R. C. Goertz. Manipulator systems development at ANL, Proceedings of the $12^{\text {th }}$ International Conference on Remote systems Technology, pp. 117-136, 1954

[18] P. S. Green, J. W. Hill, J. F. Jensen, and A. Shah. Telepresence surgery, IEEE Engineering in Medicine and Biology Magazine, vol. 4, issue 3, pp. 324-329, May-June 1995

[19] M. Green and D. J. N. Limebeer. Linear robust control, Prentice Hall, 1995

[20] B. Hannaford. Stability and performance tradeoffs in bilateral telemanipulation, Proceedings of the 1989 IEEE International Conference on Robotics and Automation, vol. 3, pp. 1764-1767, May 1989

[21] B. Hannaford. A design framework for teleoperators with kinesthetic feedback, IEEE Transactions on Robotics and Automation, vol. 5, issue 4, pp. 426-434, Aug. 1989

[22] B. Hannaford, L. Wood, D. A. McAffee, and H. Zak. Performance evaluation of a six-axis generalized force-reflecting teleoperator, IEEE Transactions on Systems Man and Cybernetics, vol. 21, issue 3, pp. 620-633, May-June, 1991

[23] K. Hashtrudi-Zaad. Design, Implementation and evaluation of stable bilateral teleoperation control architecture for enhanced telepresence, Phd thesis, Dept. of Electrical and Computer Engineering, University of British Columbia, 2000

[24] Z. Hu, S. E. Salcudean and P. D. Loewen. Robust controller design for teleoperation systems, Proceedings of the 1995 IEEE International Conference on Systems, Man and Cybernetics, vol. 3, pp. 2127-2132, Oct. 1995

[25] H. Kazerooni, T. -I. Tsay, and K. Hollerbach. A controller design framework for 
telerobotic systems, IEEE Transactions on Control Systems Technology, vol. 1, issue 1, pp. 50-62, Mar. 1993

[26] K. Kim, M. Cavusoglu and W. K. Chung. Quantitative comparison of bilateral teleoperation systems using $H_{\infty}$ framework, Proceedings of the 2004 IEEE/RSJ International Conference on Intelligent Robots and Systems, vol. 3, pp. 2229-2234, Sept-Oct, 2004

[27] W. S. Kim, B. Hannaford, and A. K. Fejczy. Force-reflection and shared compliant control in operating telemanipulators with time delay, IEEE Transactions on Robotics and Automation, vol. 8, issue 2, pp.176-185, Apr. 1992

[28] D. A. Lawrence. Stability and transparency in bilateral teleoperation, IEEE Transactions on Robotics and Automation, vol. 9, issue 5, pp. 624-637, Oct. 1993

[29] B. R. Lee, J. T. Bishoff, G. Janetschek, P. Bunyaratevej, W. Kamolpronwijit, J. A. Cadeddu, S. Ratchanon, S. O'Kelley, and L. R. Kavoussi. A novel method of surgical instruction: International telementoring, World Journal of Urology, vol. 16, pp. 367-370, 1998

[30] S. Lee and H. S. Lee. Modeling, design, and evaluation of advanced teleoperator control systems with short time delay, IEEE Transactions on Robotics and Automation, vol. 9, issue 5, pp. 607-623, Oct. 1993

[31] G. M. H. Leung, B. A. Francis, and J. Apkarian. Bilateral controller for teleoperators with time delay via $\mu$ synthesis, IEEE Transactions on Robotics and Automation, vol. 11, issue 1, pp. 105-116, Feb. 1995

[32] L. Ljung. System identification - theory for the user, Prentice-Hall, 1987 
[33] L. J. Love and W. J. Book. Force reflecting teleoperation with adaptive impedance control, IEEE Transactions on Systems, Man and Cybernetics, Part B: cybernetics, vol. 34, issue 1, pp. 159-165, Feb. 2004

[34] A. J. Madhani, G. Niemeyer, and J. K. Salisbury. The black falcon: a teleoperated surgical instrument for minimally invasive surgery, Proceedins of the 1998 International Conference on Intelligent Robots and Systems, vol. 2, pp. 936-944, Oct. 1998

[35] C. Melchiorri and A. Eusebi. Telemanipulation: System aspects and control issues. In C. Melchiorri and A. Tornambe, editors, Proceedings of International Summer School on Modeling and Control of Mechanisms and Robots, pp. 149-183. World Scientific, Bertinoro, 1996

[36] G. Niemeyer and J. J. -J.E. Slotine. Stable adaptive teleoperation, IEEE Journal of Oceanic engineering, vol. 16, issue. 1, pp. 152-162, Jan. 1991

[37] G. J. Raju, G. C. Verghese, and T. B. Sheridan. Design issues in 2-port network models of bilateral remote manipulation, Proceedings of the 1989 IEEE International Conference on Robotics and Automation, vol. 3, pp. 1316-1321, May 1989

[38] A. Rovetta, R. Sala, F. Cosmi, X. Wen, D. Sabbadini, S. Milanesi, A. Tongo, L. Angelini, A. Bejczy. The first experiment in the world of robotic telesurgery for laparoscopy carried out by means of satellites networks and optical fibres networks on $7^{\text {th }}$ July 1993, Proceedings of the IECON '93. International Conference on Industrial Electronics, Control, and Instrumentation, vol. 1, pp. 51 - 56, Nov. 1993

[39] T. B. Sheridan. Telerobotics, Automatica, vol. 25, issue. 4, pp. 487-507, 1989

[40] P. G. Shulam, S. G. Docimo, W. Saleh, C. Breitenbach, R.G. Moore, and L. Kavoussi. 
Telesurgical mentoring, Surgical Endoscopy, vol 11, pp. 1001-1005, 1997

[41] Y. Strassberg, A. A. Goldenberg and J. K. Mills. Stability analysis of a bilateral teleoperating system, ASME Jounal of Dynamic Systems, Measurement, and control, vol. 115 , pp. 419-425, 1993

[42] Y. Strassberg, A.A. Goldenberg and J. K. Mills. A new control scheme for bilateral teleoperating systems: Lyapunov stability analysis, Proceedings of the 1992 IEEE International Conference on Robotics and Automation, vol. 1, pp. 837-842, May 1992

[43] S. Tafazoli, P. D. Lawrence, and S. E. Salcudean. Identification of inertial and friction parameters for excavator arms, IEEE Transactions on Robotics and Automation, vol. 15 , issue 5, pp. 966-971, Oct. 1999

[44] S. Tøffner-Clausen, System identification and robust control, Springer, 1996

[45] X. Wang, P. X. Liu, B. Chebbi and D. Wang. Design of bilateral teleoperators for soft environments with adaptive environmental impedance estimation, Proceedings of the 2005 IEEE International Conference on Robotics and Automation, pp. 1139-1144, April 2005

[46] X. Wang, P. X. Liu and S. Wang. Robust adaptive control of teleoperators in soft environments, Proceedings of the 2005 IEEE International Conference on Mechatronics and Automation, pp. 2055-2060, July 2005

[47] J. Yan and S. E. Salcudean. Teleoperation controller design using $H_{\infty}$ optimization with application to motion-scaling, IEEE Transactions on Control Systems Technology, vol. 4, issue 3, pp. 244-258, May 1996

[48] Y. Yokokohji and T. Yoshikawa. Bilateral control of master-slave manipulators for 
ideal kinesthetic coupling - formulation and experiment, IEEE Transactions on Robotics and Automation, vol. 10, pp. 605-620, Oct. 1994

[49] G. Zames. Feedback and optimal sensitivity: Model reference transformations, multiplicative seminorms, and approximate inverses, IEEE Transactions on Automatic Control, vol. 26, issue 2, pp. 301-320, April 1981

[50] K. Zhou and J. C. Doyle. Essentials of robust control, Prentice Hall, 1998 